\title{
Asymmetric Total Synthesis of (+)-K01-0509 B; Determination of Absolute Configuration
}

\section{Supporting Information-1}

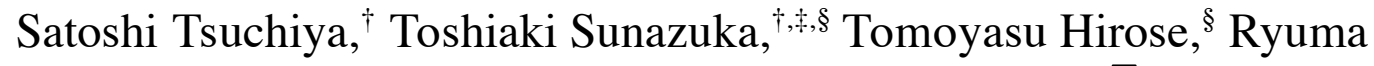
Mori, ${ }^{\dagger}$ Toshiaki Tanaka, ${ }^{\dagger}$ Masato Iwatsuki, ${ }^{\S}$ and Satoshi Omura ${ }^{*},+,, \S$

Kitasato Institute for Life Sciences \& Graduate School of Infection Control Sciences, and The Kitasato Institute, 5-9-1 Shirokane, Minato-ku, Tokyo 108-8641, Japan 


\section{A Table of Contents}

Scheme 2. Synthesis of aldehyde (-)-9

1. Synthesis of compound (+)-14; $\mathrm{p}-4$

2. Synthesis of compound (+)-I; p-7

3. Synthesis of compound (-)-15; $\mathrm{p}-9$

4. Synthesis of compound (-)-9; p-11

Table 1. Investigation of asymmetric nitroaldol reaction of (-)-9

5. Synthesis of compound (-)-8; p-13

6. Synthesis of compound (-)-11; p-15

Scheme 3. Syntheisis of cyclic guanidine (-)-6

7. Synthesis of compound (-)-7; p-17

Determination of the stereochemistry of nitroaldol product (-)-8.

8. Synthesis of compound (+)-II; p-19

9. Synthesis of compound (-)-III; p-21

Scheme 3. Synthesis of cyclic guanidine (-)-6

10. Synthesis of compound (-)-6; p-23

Scheme 4. Synthesis of (+)-(5R, 4'R)-K01-0509 B (5).

11. Synthesis of compound (-)-IV; p-25

12. Synthesis of compound (-)-18; p-27

13. Synthesis of compound (-)-V; p-29

14. Synthesis of compound (-)-19; p-31

15. Synthesis of compound (-)-VI; $p-33$

16. Synthesis of compound (+)-(+)-(5R, 4'R)-K01-0509 B (5); p-35

Scheme 5. Synthesis of (5R, 4'S)-K01-0509 B (10).

17. Synthesis of compound (-)-20; p-36

18. Synthesis of compound (+)-21; p-38

19. Synthesis of compound (+)-VII; $\mathrm{p}-40$

20. Synthesis of compound (+)-VIII; $\mathrm{p}-41$ 
21. Synthesis of compound (+)-IX; p-42

22. Synthesis of compound (+)-22; $\mathrm{p}-44$

23. Synthesis of compound (+)-X; p-46

24. Synthesis of compound (-)-(5R, 4'S)-K01-0509 B (10); p-47 


\section{Experimental section}

\section{Methods and Material}

\section{General.}

Unless otherwise noted, all other commercially obtained reagents and solvents were used as received. All reactions were monitored by thin-layer chromatography using E. Merck silica gel 60 F254 pre-coated plates $(0.25 \mathrm{~mm})$. Flash chromatography was performed with indicated solvents using E. Merck silica gel 60 (partical size 0.040-0.063). ${ }^{1} \mathrm{H}$ NMR spectra were recorded on JEOL JNM-EX270 (270 MHz), VXR-300 (300 MHz), VXR-300 (400 MHz), and ${ }^{13} \mathrm{C}$ NMR spectra were recorded on JEOL JNM-EX270 (67.5 MHz), VXR-300 (75 MHz), VXR-300 (100 MHz). Chemical shifts are reported relative to internal chloroform $\left({ }^{1} \mathrm{H} ; \delta=7.26,{ }^{13} \mathrm{C}, \delta=77.1\right)$, methanol $\left({ }^{1} \mathrm{H}, \delta=3.31,{ }^{13} \mathrm{C}, \delta=49.2\right), \mathrm{H}_{2} \mathrm{O}\left({ }^{1} \mathrm{H} ; \delta=4.76\right)$, pyridine $\left({ }^{1} \mathrm{H} ; \delta=8.73,7.58,7.21,{ }^{13} \mathrm{C}\right.$; $\delta=149.9,135.5,123.5)$ as indicated. Melting points were obtained on a YANAGIMOTO MICRO MELTING APPARATUS and are uncorrected. Infrared spectra were recorded on a Horiba FT-210 spectrometer. High resolution mass spectra were acquired on a JEOL JMS-AX505 HA Mass spectrometer. Low resolution mass spectra were acquired on a JEOL JMS-DX300 Mass spectrometer. 


\section{Preparative Procedures.}

\section{Scheme 2. Synthesis of aldehyde (-)-9}

(R)-7-(tert-Butyldimethylsiloxy)heptane-1,3-diol (+)-14

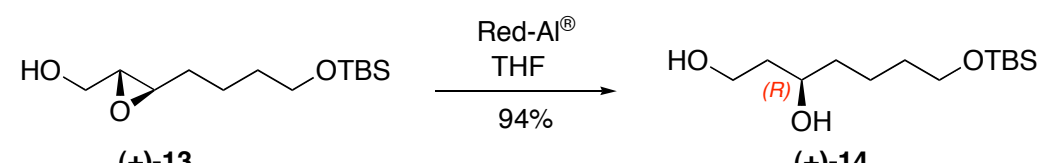

$(+)-13$

$(+)-14$

To a solution of epoxy alcohol (+)-13 (4.96 g, $19.0 \mathrm{mmol})$ in $\mathrm{CH}_{2} \mathrm{Cl}_{2}(63.5 \mathrm{~mL})$, Red-Al ${ }^{\circledR}$ (in toluene $65 \mathrm{wt} \%, 14.8 \mathrm{~mL}, 47.6 \mathrm{mmol}$ ) was added over $13 \mathrm{~min}$ at $-20^{\circ} \mathrm{C}$ under Ar. After stirring for $12 \mathrm{~min}$, the solution was warmed to $0^{\circ} \mathrm{C}$ and stirred for $5 \mathrm{hr}$ at the temparature. The reaction mixture was added sat. aq. Rochelle's salt solution (20 $\mathrm{mL})$, then the mixture was stirred for $30 \mathrm{~min}$ at r.t.. $\mathrm{CHCl}_{3}(100 \mathrm{~mL})$ and sat. aq. Rochelle' salt solution (100 mL) were added to the reaction solution, and the organic layer was separated, and the aqueous layer was extracted with $\mathrm{CHCl}_{3}(200 \mathrm{~mL} \mathrm{X} \mathrm{2).} \mathrm{The}$ combined organic extracts were washed with sat. Rochelle's salt aq. (200 mL X 2) and dried over $\mathrm{Na}_{2} \mathrm{SO}_{4}$, filtered, and evaporated under reduced pressure. Purification by flash chromatography on silica gel (Hexane:EtOAc=1:1) provided the title compound (+)-14 (4.70 g, 94\%) as a colorless oil.

$\mathbf{R} \boldsymbol{f}=0.29$ (Silica gel, Hexane $:$ EtOAc $=1: 1$ )

$[\alpha]_{\mathbf{D}}{ }^{24}+0.72\left(c 1.0, \mathrm{CHCl}_{3}\right)$

IR $(\mathrm{KBr}) \vee \mathrm{cm}^{-1}$

$3352(\mathrm{OH})$

${ }^{1} \mathrm{H}$-NMR (270 MHz, $\mathrm{CDCl}_{3}$ )

$\delta$ : 3.93-3.80 (complex m, 2H, 1- $\mathrm{H}_{2}$ ), 3.93-3.80 (complex m, 1H, 3-H),

$3.62\left(\mathrm{t}, J=5.9 \mathrm{~Hz}, 2 \mathrm{H}, 7-\mathrm{H}_{2}\right), 1.75-1.65\left(\mathrm{~m}, 2 \mathrm{H}, 2-\mathrm{H}_{2}\right)$,

1.60-1.39 (complex m, 2H, 4- $\mathrm{H}_{2}$ ), 1.60-1.39 (complex m, 2H, 5- $\mathrm{H}_{2}$ ),

1.60-1.39 (complex m, 2H, 6- $\left.\mathrm{H}_{2}\right), 0.89$ (s, 9H, 7-O-Si[ $\left.\left.\left(\mathrm{CH}_{3}\right)_{2}\right] \mathrm{C}\left(\mathrm{CH}_{3}\right)_{3}\right)$,

$0.05\left(\mathrm{~s}, 6 \mathrm{H}, 7-\mathrm{O}-\mathrm{Si}\left[\left(\mathrm{CH}_{3}\right)_{2}\right] \mathrm{C}\left(\mathrm{CH}_{3}\right)_{3}\right)$

${ }^{13} \mathrm{C}-\mathrm{NMR}\left(67.5 \mathrm{MHz} \mathrm{CDCl}_{3}\right)$

$\delta$ : 71.3 (C-3), 63.1 (C-7), 61.0 (C-1), 38.2 (C-2), 37.3 (C-4), 32.6 (C-6),

25.8 (3C, 7-O-Si $\left.\left[\left(\mathrm{CH}_{3}\right)_{2}\right] \mathrm{C}\left(\mathrm{CH}_{3}\right)_{3}\right), 18.2\left(1 \mathrm{C}, 7-\mathrm{O}-\mathrm{Si}\left[\left(\mathrm{CH}_{3}\right)_{2}\right] \underline{\mathrm{C}}\left(\mathrm{CH}_{3}\right)_{3}\right), 21.7,(\mathrm{C}-5)$,

$-5.4\left(2 \mathrm{C}, 7-\mathrm{O}-\mathrm{Si}\left[\left(\mathrm{CH}_{3}\right)_{2}\right] \mathrm{C}\left(\mathrm{CH}_{3}\right)_{3}\right)$, 
HR-MS (FAB, NBA matrix)

$\mathbf{m} / \mathbf{z} 263.2048[\mathrm{M}+\mathrm{Na}]^{+}$, Calcd for $\mathrm{C}_{13} \mathrm{H}_{31} \mathrm{O}_{3} \mathrm{Si} 263.2042[\mathrm{M}+\mathrm{Na}]$ 
- $\quad(2 S, 4 R)-4-\left[4^{\prime}\right.$-(tert-Butyldimethylsiloxy)butyl]-2-(p-methoxyphenyl)-1,3-dioxane $(+)-\mathbf{I}$

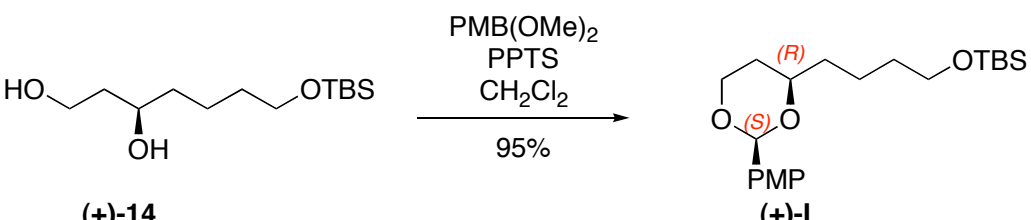

To a solution of diol (+)-14 (4.22 g, $16.1 \mathrm{mmol})$ in $\mathrm{CH}_{2} \mathrm{Cl}_{2}(161 \mathrm{~mL})$ was added p-anisaldehyde dimethylacetal $(3.01 \mathrm{~mL}, 17.7 \mathrm{mmol})$, PPTS $(202 \mathrm{mg}, 0.805 \mathrm{mmol})$ at $0^{\circ} \mathrm{C}$ under Ar. The mixture was stirred for $25 \mathrm{~min}$ and then quenched with sat. aq. $\mathrm{NaHCO}_{3}(30 \mathrm{~mL})$. Then two layers were separated and the aqueous layer was extracted with $\mathrm{CHCl}_{3}(100 \mathrm{~mL} \times 3)$. The combined organic extracts were washed with brine (50 $\mathrm{mL}$ ) and dried over $\mathrm{Na}_{2} \mathrm{SO}_{4}$, filtered, and evaporated under reduced pressure. Purification by flash chromatography on silica gel (Hexane : EtOAc $=5: 1$ ) provided the title compound (+)-I (5.79 $\mathrm{g}, 95 \%)$ as a colorless oil.

$\mathbf{R} \boldsymbol{f}=0.54$ (Silica gel, Hexane $:$ EtOAc $=2: 1$ )

$[\alpha]_{\mathrm{D}}{ }^{23}+18.2\left(c 2.8, \mathrm{CHCl}_{3}\right)$

IR $(\mathrm{KBr}) \vee \mathrm{cm}^{-1}$

1616, 1517 (Ar-C), 1249, 1105, 1037 (C-O-C)

\section{${ }^{1} \mathrm{H}-\mathrm{NMR}\left(270 \mathrm{MHz}, \mathrm{CDCl}_{3}\right)$}

$\delta: 7.43\left(\mathrm{~d}, J=8.7 \mathrm{~Hz}, 2 \mathrm{H}, 2-\underline{\mathrm{Ph}}-\mathrm{OCH}_{3}\right), 6,89\left(\mathrm{~d}, J=8.7 \mathrm{~Hz}, 2 \mathrm{H}, 2-\underline{\mathrm{Ph}}-\mathrm{OCH}_{3}\right)$,

$5.47(\mathrm{~s}, 1 \mathrm{H}, 2-\mathrm{H}), 4.25(\mathrm{dd}, 1 \mathrm{H}, J=4.6,11.4 \mathrm{~Hz}, 6-\mathrm{H})$,

$3.93(\mathrm{dt}, J=2.6,11.4 \mathrm{~Hz}, 1 \mathrm{H}, 6-\mathrm{H}), 3.86-3.80$ (m, 1H, 4-H), 3.79 (s, 3H, 2-Ph-OC $\underline{\mathrm{H}}_{3}$ ),

$3.64\left(\mathrm{t}, J=5.93 \mathrm{~Hz}, 2 \mathrm{H}, 4^{\prime}-\mathrm{H}_{2}\right), 1.87-1.66\left(\mathrm{~m}, 2 \mathrm{H}, 5-\mathrm{H}_{2}\right)$,

1.56-1.48 (complex m, 2H, 1'- $\mathrm{H}_{2}$ ), 1.56-1.48 (complex m, 2H, 2'- $\mathrm{H}_{2}$ ),

1.56-1.48 (complex m, 2H, 3'- $\left.\mathrm{H}_{2}\right), 0.92$ (s, 9H, 4'-O-Si $\left.\left[\left(\mathrm{CH}_{3}\right)_{2}\right] \mathrm{C}\left(\mathrm{CH}_{3}\right)_{3}\right)$,

0.07 (s, 6H, 4'-O-Si[ $\left.\left.\left(\mathrm{C}_{3}\right)_{2}\right] \mathrm{C}\left(\mathrm{CH}_{3}\right)_{3}\right)$

${ }^{13} \mathrm{C}-\mathrm{NMR}\left(67.5 \mathrm{MHz} \mathrm{CDCl}_{3}\right)$

$\delta$ : 159.7 (1C, 2- $\left.\underline{\mathrm{Ph}}-\mathrm{OCH}_{3}\right), 131.5\left(1 \mathrm{C}, 2-\underline{\mathrm{Ph}}-\mathrm{OCH}_{3}\right), 127.4$ (2C, 2- $\left.\underline{\mathrm{Ph}}-\mathrm{OCH}_{3}\right)$,

113.4 (2C, 2- $\left.\underline{\mathrm{Ph}}-\mathrm{OCH}_{3}\right), 100.9$ (C-2), 77.3 (C-4), 66.9 (C-4'), 62.9 (C-6),

55.1 (1C, 2-Ph- $\mathrm{OCH}_{3}$ ), 35.7 (C-1'), 32.7 (C-3'), 31.2 (C-5),

25.9 (3C, 4'-O-Si $\left.\left[\left(\mathrm{CH}_{3}\right)_{2}\right] \mathrm{C}\left(\mathrm{CH}_{3}\right)_{3}\right), 21.2,\left(\mathrm{C}-2^{\prime}\right), 18.3$ (1C, 4'-O-Si $\left.\left[\left(\mathrm{CH}_{3}\right)_{2}\right] \underline{\mathrm{C}}\left(\mathrm{CH}_{3}\right)_{3}\right)$,

$-5.3\left(2 \mathrm{C}, 4^{\prime}-\mathrm{O}-\mathrm{Si}\left[\left(\mathrm{CH}_{3}\right)_{2}\right] \mathrm{C}\left(\mathrm{CH}_{3}\right)_{3}\right)$

HR-MS (FAB, NBA matrix) 
$\mathbf{m} / \mathbf{z} 381.2463[\mathrm{M}+\mathrm{H}]^{+}$, Calcd for $\mathrm{C}_{21} \mathrm{H}_{37} \mathrm{O}_{4} \mathrm{Si} 381.2461[\mathrm{M}+\mathrm{H}]$ 
- (R)-7-(tert-Butyldimethylsiloxy)-3-(p-methoxybenzyloxy)heptanol (-)-15

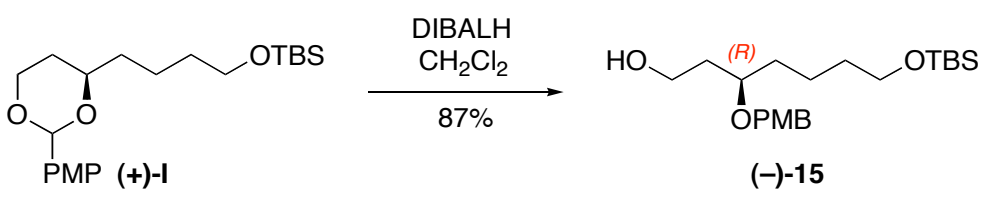

To a solution of (+)-I $(5.63 \mathrm{~g}, 14.8 \mathrm{mmol})$ in $\mathrm{CH}_{2} \mathrm{Cl}_{2}(148 \mathrm{~mL})$ was dropwised DIBAL-H solution in $\mathrm{CH}_{2} \mathrm{Cl}_{2}(1.0 \mathrm{M}$ in hexane, $44.4 \mathrm{~mL}, 44.4 \mathrm{mmol})$ at $-5^{\circ} \mathrm{C}$ under $\mathrm{Ar}$ over $10 \mathrm{~min}$. After stirring for $5 \mathrm{~min}$, the solution was diluted with $\mathrm{CHCl}_{3}(200 \mathrm{~mL})$, quenched by addition of sat. aq. Rochelle's salt solution $(150 \mathrm{~mL})$. The organic layer was separated, and the aqueous layer was extracted with $\mathrm{CHCl}_{3}(300 \mathrm{~mL} \mathrm{X} \mathrm{3})$. The combined organic extracts were washed with sat. aq. Rochelle's salt solution ( $200 \mathrm{~mL} \mathrm{X}$ 2), brine $(200 \mathrm{~mL})$, dried over $\mathrm{Na}_{2} \mathrm{SO}_{4}$, filtered, and evaporated under reduced pressure. Purification by flash chromatography on silica gel (Hexane : EtOAc $=5: 1$ to $3: 1$ ) provided the title compound (-)-15(4.90 g, 87\%) as a colorless oil.

$\mathbf{R} \boldsymbol{f}=0.27$ (Silica gel, Hexane $:$ EtOAc $=2: 1$ )

$[\alpha]_{\mathbf{D}}{ }^{24}-24.5\left(c 0.52, \mathrm{CHCl}_{3}\right)$

IR $(\mathrm{KBr}) \vee \mathrm{cm}^{-1}$

$3429(\mathrm{OH}), 1614,1514(\mathrm{Ar}-\mathrm{C})$

\section{${ }^{1} \mathrm{H}-\mathrm{NMR}\left(270 \mathrm{MHz} \mathrm{CDCl}_{3}\right)$}

$\delta: 7.26\left(\mathrm{~d}, J=8.6 \mathrm{~Hz}, 2 \mathrm{H}, 3-\mathrm{O}-\mathrm{CH}_{2}-\mathrm{Ph}-\mathrm{OCH}_{3}\right)$,

$6.87\left(\mathrm{~d}, J=8.6 \mathrm{~Hz}, 2 \mathrm{H}, 3-\mathrm{O}-\mathrm{CH}_{2}-\mathrm{Ph}-\mathrm{OCH}_{3}\right)$,

$4.53\left(\mathrm{~d}, J=10.9 \mathrm{~Hz}, 1 \mathrm{H}, 3-\mathrm{O}-\mathrm{CH}_{2}-\mathrm{Ph}-\mathrm{OCH}_{3}\right)$,

$4.41\left(\mathrm{~d}, J=10.9 \mathrm{~Hz}, 1 \mathrm{H}, 3-\mathrm{O}-\mathrm{C}_{2}-\mathrm{Ph}-\mathrm{OCH}_{3}\right), 3.80$ (s, 3H, 3-O-CH

3.78-3.67 (complex m, 1H, 1-H), 3.78-3.67 (complex m, 2H, 3-H),

$3.61\left(\mathrm{t}, J=6.3 \mathrm{~Hz}, 2 \mathrm{H}, 7-\mathrm{H}_{2}\right), 1.82-1.62\left(\mathrm{~m}, 2 \mathrm{H}, 2-\mathrm{H}_{2}\right), 1.82-1.62\left(\mathrm{~m}, 2 \mathrm{H}, 4-\mathrm{H}_{2}\right)$,

1.58-1.49 (m, 2H, 6- $\left.\mathrm{H}_{2}\right), 1.44-1.35\left(\mathrm{~m}, 2 \mathrm{H}, 5-\mathrm{H}_{2}\right), 0.90$ (s, 9H, 7-O-Si[ $\left.\left[\left(\mathrm{CH}_{3}\right)_{2}\right] \mathrm{C}\left(\mathrm{C}_{3}\right)_{3}\right)$, $0.05\left(\mathrm{~s}, 6 \mathrm{H}, 7-\mathrm{O}-\mathrm{Si}\left[\left(\mathrm{C}_{\underline{3}}\right)_{2}\right] \mathrm{C}\left(\mathrm{CH}_{3}\right)_{3}\right)$

${ }^{13} \mathrm{C}-\mathrm{NMR}\left(67.5 \mathrm{MHz}, \mathrm{CDCl}_{3}\right)$

$\delta$ : 159.2 (1C, 3-O-CH$\left.-\underline{\mathrm{Ph}}-\mathrm{OCH}_{3}\right), 130.4$ (1C, 3-O- $\left.\mathrm{CH}_{2}-\underline{\mathrm{Ph}}-\mathrm{OCH}_{3}\right)$, 129.4 (2C, 3-O-CH$\left.-\mathrm{Ph}-\mathrm{OCH}_{3}\right), 113.8$ (2C, 3-O-CH$\left.-\mathrm{Ph}-\mathrm{OCH}_{3}\right), 78.2$ (C-3),

70.6 (1C, 3-O- $\left.\underline{\mathrm{CH}}_{2}-\mathrm{Ph}-\mathrm{OCH}_{3}\right), 62.9$ (C-7), 60.7 (C-1), $55.2\left(1 \mathrm{C}, 3-\mathrm{O}-\mathrm{CH}_{2}-\mathrm{Ph}-\mathrm{OCH}_{3}\right)$, 35.8 (C-2), 33.3 (C-4), 32.9 (C-6), 25.9 (3C, 7-O-Si $\left.\left[\left(\mathrm{CH}_{3}\right)_{2}\right] \mathrm{C}\left(\mathrm{CH}_{3}\right)_{3}\right), 21.5$, (C-5), $18.3\left(1 \mathrm{C}, 7-\mathrm{O}-\mathrm{Si}\left[\left(\mathrm{CH}_{3}\right)_{2}\right] \underline{\mathrm{C}}\left(\mathrm{CH}_{3}\right)_{3}\right),-5.3\left(2 \mathrm{C}, 7-\mathrm{O}-\mathrm{Si}\left[\left(\mathrm{CH}_{3}\right)_{2}\right] \mathrm{C}\left(\mathrm{CH}_{3}\right)_{3}\right)$

\section{HR-MS (FAB, NBA matrix)}


$\mathbf{m} / \mathbf{z} 405.2443[\mathrm{M}+\mathrm{Na}]^{+}$, Calcd for $\mathrm{C}_{21} \mathrm{H}_{38} \mathrm{O}_{4} \mathrm{SiNa} 405.2437[\mathrm{M}+\mathrm{Na}]$ 
- (R)-7-(tert-Butyldimethylsiloxy)-3-(p-methoxybenzyloxy)heptanal (-)-9

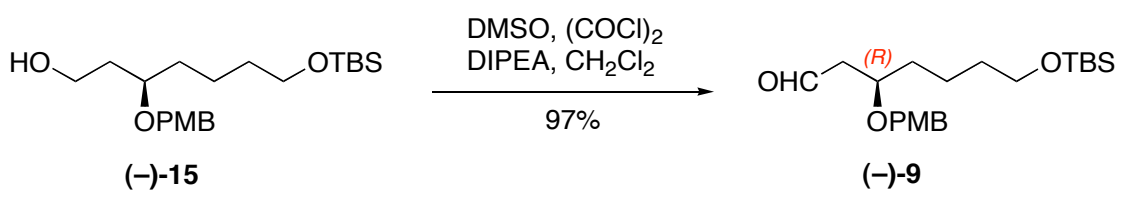

To a solution of DMSO $(301 \mu \mathrm{L}, 4.24 \mathrm{mmol})$ in $\mathrm{CH}_{2} \mathrm{Cl}_{2}(7.0 \mathrm{~mL})$ was dropwised oxalyl chloride $(182 \mu \mathrm{L}, 2.09 \mathrm{mmol})$ at $-78^{\circ} \mathrm{C}$ under Ar. The solution was stirred over $30 \mathrm{~min}$, then dropwised alcohol (-)-15 (498.6 mg, $1.30 \mathrm{mmol})$ in $\mathrm{CH}_{2} \mathrm{Cl}_{2}(6.0 \mathrm{~mL})$ at the temparature. After sttiring for $30 \mathrm{~min}$ at $-78^{\circ} \mathrm{C}$, the solution was added DIPEA (1.36 $\mathrm{mL}, 7.82 \mathrm{mmol}$ ), then warmed to $0^{\circ} \mathrm{C}$. The mixture was stirred for $10 \mathrm{~min}$, and quenched with sat. $\mathrm{NH}_{4} \mathrm{Cl}$ aq. $(10 \mathrm{~mL})$. Then the organic layer was separated, and the aqueous layer was extracted with $\mathrm{CHCl}_{3}(15 \mathrm{~mL}$ x 3$)$. The combined organic extracts were dried over $\mathrm{Na}_{2} \mathrm{SO}_{4}$, filtered, and evaporated under reduced pressure. Purification by flash chromatography on silica gel (Hexane : EtOAc $=5: 1$ ) provided the title compound (-)-9 (479 mg, 97\%) as colorless oil.

$\mathbf{R} \boldsymbol{f}=0.54$ (Silica gel, Hexane $:$ EtOAc $=2: 1$ )

$[\alpha]_{\mathrm{D}}{ }^{22}-10.1\left(c 0.21, \mathrm{CHCl}_{3}\right)$

IR $(\mathrm{KBr}) \vee \mathrm{cm}^{-1}$

$1726(\mathrm{C}=\mathrm{O}), 1614,1514(\mathrm{Ar}-\mathrm{C})$

\section{${ }^{1} \mathrm{H}-\mathrm{NMR}\left(270 \mathrm{MHz}^{\mathrm{CDCl}}{ }_{3}\right)$}

$\delta: 9.78(\mathrm{t}, J=2.6 \mathrm{~Hz}, 1 \mathrm{H}, 1-\mathrm{H}), 7.25\left(\mathrm{~d}, J=2.0 \mathrm{~Hz}, 1 \mathrm{H}, 3-\mathrm{O}-\mathrm{CH}_{2}-\underline{\mathrm{Ph}}-\mathrm{OCH}_{3}\right)$,

$7.22\left(\mathrm{~d}, J=2.0 \mathrm{~Hz}, 1 \mathrm{H}, 3-\mathrm{O}-\mathrm{CH}_{2}-\mathrm{Ph}-\mathrm{OCH}_{3}\right)$,

$6.88\left(\mathrm{~d}, J=2.0 \mathrm{~Hz}, 1 \mathrm{H}, 3-\mathrm{O}-\mathrm{CH}_{2}-\mathrm{Ph}-\mathrm{OCH}_{3}\right)$,

$6.85\left(\mathrm{~d}, J=2.0 \mathrm{~Hz}, 1 \mathrm{H}, 3-\mathrm{O}-\mathrm{CH}_{2}-\mathrm{Ph}-\mathrm{OCH}_{3}\right)$,

$4.50\left(\mathrm{~d}, J=10.9 \mathrm{~Hz}, 1 \mathrm{H}, 3-\mathrm{O}-\mathrm{CH}_{2}-\mathrm{Ph}-\mathrm{OCH}_{3}\right)$,

$4.44\left(\mathrm{~d}, J=10.9 \mathrm{~Hz}, 1 \mathrm{H}, 3-\mathrm{O}-\mathrm{C}_{2}-\mathrm{Ph}-\mathrm{OCH}_{3}\right), 3.97-3.86(\mathrm{~m}, 1 \mathrm{H}, 3-\mathrm{H})$,

$3.80\left(\mathrm{~s}, 3 \mathrm{H}, 3-\mathrm{O}-\mathrm{CH}_{2}-\mathrm{Ph}-\mathrm{OC}_{3}\right), 3.61\left(\mathrm{t}, J=5.9 \mathrm{~Hz}, 2 \mathrm{H}, 7-\mathrm{H}_{2}\right)$,

2.66 (ddd, $J=2.6,7,3,16.2 \mathrm{~Hz}, 1 \mathrm{H}, 2-\mathrm{H}), 2.54$ (ddd, $J=2.6,5.0,16.2 \mathrm{~Hz}, 1 \mathrm{H}, 2-\mathrm{H}$ ),

1.76-1.60 (m, 2H, 6- $\mathrm{H}_{2}$ ), 1.55-1.37 (complex m, 2H, 4- $\mathrm{H}_{2}$ ),

1.55-1.37 (complex m, 2H, 5- $\mathrm{H}_{2}$ ),

0.90 (s, 9H, 7-O-Si[( $\left.\left.\left(\mathrm{CH}_{3}\right)_{2}\right] \mathrm{C}\left(\mathrm{CH}_{3}\right)_{3}\right), 0.05\left(\mathrm{~s}, 6 \mathrm{H}, 7-\mathrm{O}-\mathrm{Si}\left[\left(\mathrm{C}_{3}\right)_{2}\right] \mathrm{C}\left(\mathrm{CH}_{3}\right)_{3}\right)$

${ }^{13} \mathrm{C}-\mathrm{NMR}$ (67.5 $\left.\mathrm{MHz}, \mathrm{CDCl}_{3}\right)$

$\delta$ : 201.6 (C-1), 159.2 (1C, 3-O-CH $\left.-\underline{\mathrm{Ph}}-\mathrm{OCH}_{3}\right), 130.1$ (1C, 3-O-CH$\left.-\underline{\mathrm{Ph}}-\mathrm{OCH}_{3}\right)$, 129.3 (2C, 3-O- $\left.\mathrm{CH}_{2}-\underline{\mathrm{Ph}}-\mathrm{OCH}_{3}\right), 113.8$ (2C, 3-O- $\left.\mathrm{CH}_{2}-\underline{\mathrm{Ph}}-\mathrm{OCH}_{3}\right), 73.9$ (C-3), 
70.9 (1C, 3-O- $\left.\mathrm{CH}_{2}-\mathrm{Ph}-\mathrm{OCH}_{3}\right), 62.8(\mathrm{C}-7), 55.2$ (1C, 3-O- $\left.\mathrm{CH}_{2}-\mathrm{Ph}-\mathrm{OCH}_{3}\right), 48.3(\mathrm{C}-2)$,

34.1 (C-4), 32.7 (C-6), 25.9 (3C, 7-O-Si[ $\left.\left.\left(\mathrm{CH}_{3}\right)_{2}\right] \mathrm{C}\left(\mathrm{CH}_{3}\right)_{3}\right), 21.3$, (C-5),

$18.3\left(1 \mathrm{C}, 7-\mathrm{O}-\mathrm{Si}\left[\left(\mathrm{CH}_{3}\right)_{2}\right] \underline{\mathrm{C}}\left(\mathrm{CH}_{3}\right)_{3}\right),-5.3\left(2 \mathrm{C}, 7-\mathrm{O}-\mathrm{Si}\left[\left(\mathrm{CH}_{3}\right)_{2}\right] \mathrm{C}\left(\mathrm{CH}_{3}\right)_{3}\right)$

HR-MS (FAB, NBA matrix)

$\mathbf{m} / \mathbf{z} 403.2289[\mathrm{M}+\mathrm{Na}]^{+}$, Calcd for $\mathrm{C}_{21} \mathrm{H}_{36} \mathrm{O}_{4} \mathrm{SiNa} 403.2281[\mathrm{M}+\mathrm{Na}]$ 


\section{Table 1. Investigation of asymmetric nitroaldol reaction of (-)-9}

- $\quad(2 S, 4 R)-8$-(tert-Butyldimethylsiloxy)-4-( $p$-methoxybenzyloxy)-1-nitrooctan-2-ol $(-)-(8)$

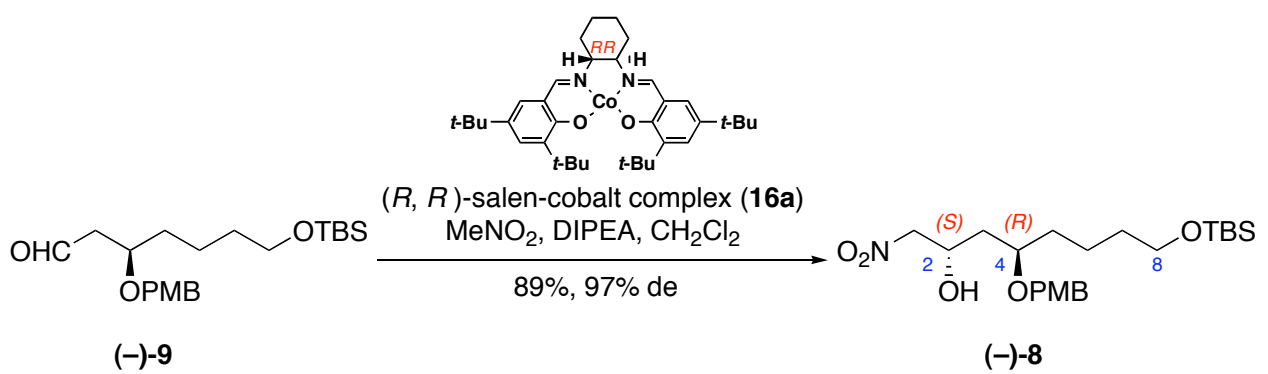

To a solution of aldehyde (-)-9 $(955 \mathrm{mg}, 2.51 \mathrm{mmol})$ in $\mathrm{CH}_{2} \mathrm{Cl}_{2}(21.0 \mathrm{~mL})$ was added $(R, R)$-salen-cobalt complex $\mathbf{1 6 a}(152 \mathrm{mg}, 0.25 \mathrm{mmol})$ and $\mathrm{MeNO}_{2}(4.20 \mathrm{~mL}, 100.4$ $\mathrm{mmol})$ at room temparature. Then the solution was cooled to $-40^{\circ} \mathrm{C}$ and added DIPEA (1.09 mL, $6.27 \mathrm{mmol}$ ). After stirring for $36 \mathrm{hr}$, the reaction was quenched with sat. $\mathrm{NH}_{4} \mathrm{Cl}$ aq. (10.0 mL), then the aqueous layer was extracted with $\mathrm{CHCl}_{3}(20 \mathrm{~mL} \times 3)$. The combined organic extracts were washed with brine $(20 \mathrm{~mL})$, dried over $\mathrm{Na}_{2} \mathrm{SO}_{4}$, filtered, and evaporated under reduced pressure. Purification by flash chromatography on silica gel (Hexane : EtOAc = $5: 1)$ provided the title compound (-)-8 (989 mg, 89\%, $97 \%$ de ) as a colorless oil.

The de of desired compound (-)-8 was determined with HPLC (CHIRALCEL OD $4.6 \phi \times 250 \mathrm{~mm}$, hexane : 2-propanol = $99: 1,0.9 \mathrm{~mL} / \mathrm{min}, 25{ }^{\circ} \mathrm{C}, 210 \mathrm{~nm}$ )

$\mathbf{R} \boldsymbol{f}=0.45$ (Silica gel, Hexane $:$ EtOAc $=2: 1$ )

$[\alpha]_{\mathbf{D}}{ }^{25}-32.0\left(c 0.86, \mathrm{CHCl}_{3}\right)$

IR $(\mathrm{KBr}) \vee \mathrm{cm}^{-1}$

3433 (O-H), 1612, 1514 (Ar-C), $1554\left(\mathrm{NO}_{2}\right)$

${ }^{1} \mathrm{H}-\mathrm{NMR}\left(400 \mathrm{MHz}, \mathrm{CDCl}_{3}\right)$

$\delta: 7.25\left(\mathrm{~d}, J=8.6 \mathrm{~Hz}, 2 \mathrm{H}, 4-\mathrm{O}-\mathrm{CH}_{2}-\mathrm{Ph}-\mathrm{OCH}_{3}\right)$,

$6.89\left(\mathrm{~d}, J=8.6 \mathrm{~Hz}, 2 \mathrm{H}, 4-\mathrm{O}-\mathrm{CH}_{2}-\mathrm{Ph}-\mathrm{OCH}_{3}\right)$,

$4.55\left(\mathrm{~d}, J=11.2 \mathrm{~Hz}, 1 \mathrm{H}, 4-\mathrm{O}-\mathrm{CH}_{2}-\mathrm{Ph}-\mathrm{OCH}_{3}\right)$,

4.39 (d, $\left.J=11.2 \mathrm{~Hz}, 1 \mathrm{H}, 4-\mathrm{O}-\mathrm{CH}_{2}-\mathrm{Ph}-\mathrm{OCH}_{3}\right), 4.60-4.48(\mathrm{~m}, 1 \mathrm{H}, 2-\mathrm{H})$,

$4.37\left(\mathrm{dd}, J=5.0,12.5 \mathrm{~Hz}, 1 \mathrm{H}, 1-\underline{\mathrm{H}}_{2}\right), 4.33\left(\mathrm{dd}, J=7.0,12.5 \mathrm{~Hz}, 1 \mathrm{H}, 1-\underline{\mathrm{H}}_{2}\right)$,

$3.80\left(\mathrm{~s}, 3 \mathrm{H}, 4-\mathrm{O}-\mathrm{CH}_{2}-\mathrm{Ph}-\mathrm{OC}_{3}\right), 3.75-3.67(\mathrm{~m}, 1 \mathrm{H}, 4-\mathrm{H}), 3.62\left(\mathrm{t}, J=5.9 \mathrm{~Hz}, 2 \mathrm{H}, 8-\mathrm{H}_{2}\right)$,

$1.72\left(\mathrm{ddd}, J=3.0,9,0,14.5 \mathrm{~Hz}, 1 \mathrm{H}, 3-\underline{\mathrm{H}}_{2}\right), 1.62\left(\mathrm{ddd}, J=3.0,8.5,14.5 \mathrm{~Hz}, 1 \mathrm{H}, 3-\underline{\mathrm{H}}_{2}\right)$,

1.78-1.62 (m, 2H, 5- $\left.\mathrm{H}_{2}\right), 1.59-1.49\left(\mathrm{~m}, 2 \mathrm{H}, 7-\mathrm{H}_{2}\right), 1.43-1.33\left(\mathrm{~m}, 2 \mathrm{H}, 6-\mathrm{H}_{2}\right)$, 
0.90 (s, 9H, 8-O-Si[ $\left.\left.\left(\mathrm{CH}_{3}\right)_{2}\right] \mathrm{C}\left(\mathrm{C}_{3}\right)_{3}\right), 0.05$ (s, 6H, 8-O-Si $\left.\left[\left(\mathrm{C}_{3}\right)_{2}\right] \mathrm{C}\left(\mathrm{CH}_{3}\right)_{3}\right)$

\section{${ }^{13} \mathrm{C}-\mathrm{NMR}\left(67.5 \mathrm{MHz}, \mathrm{CDCl}_{3}\right.$ ), major isomer was indicated.}

$\delta$ : 159.4 (1C, 4-O- $\left.\mathrm{CH}_{2}-\mathrm{Ph}-\mathrm{OCH}_{3}\right), 129.9$ (1C, 4-O- $\left.\mathrm{CH}_{2}-\mathrm{Ph}-\mathrm{OCH}_{3}\right)$,

129.6 (2C, 4-O- $\left.\mathrm{CH}_{2}-\mathrm{Ph}-\mathrm{OCH}_{3}\right), 113.9$ (2C, 4-O- $\left.\mathrm{CH}_{2}-\mathrm{Ph}-\mathrm{OCH}_{3}\right), 80.6(\mathrm{C}-1), 75.3$ (C-4),

70.9 (1C, 3-O- $\left.\underline{\mathrm{CH}}_{2}-\mathrm{Ph}-\mathrm{OCH}_{3}\right), 66.2(\mathrm{C}-2), 62.8$ (C-8), $55.2\left(1 \mathrm{C}, 4-\mathrm{O}-\mathrm{CH}_{2}-\mathrm{Ph}-\mathrm{OCH}_{3}\right)$,

36.8 (C-3), 33.0 (C-5), 32.8 (C-7), 25.9 (3C, 8-O-Si $\left.\left[\left(\mathrm{CH}_{3}\right)_{2}\right] \mathrm{C}\left(\mathrm{CH}_{3}\right)_{3}\right), 21.4$, (C-6),

$18.3\left(1 \mathrm{C}, 8-\mathrm{O}-\mathrm{Si}\left[\left(\mathrm{CH}_{3}\right)_{2}\right] \underline{\mathrm{C}}\left(\mathrm{CH}_{3}\right)_{3}\right),-5.3\left(2 \mathrm{C}, 8-\mathrm{O}-\mathrm{Si}\left[\left(\mathrm{CH}_{3}\right)_{2}\right] \mathrm{C}\left(\mathrm{CH}_{3}\right)_{3}\right)$

HR-MS (FAB, NBA matrix)

$\mathbf{m} / \mathbf{z} 464.2458[\mathrm{M}+\mathrm{Na}]^{+}$, Calcd for $\mathrm{C}_{22} \mathrm{H}_{39} \mathrm{NO}_{6} \mathrm{SiNa} 464.2444[\mathrm{M}+\mathrm{Na}]$ 
- $\quad(2 S, 4 R)-8$-(tert-Butyldimethylsiloxy)-4-( $p$-methoxybenzyloxy)-1-nitrooctan-2-ol $(-)-11$

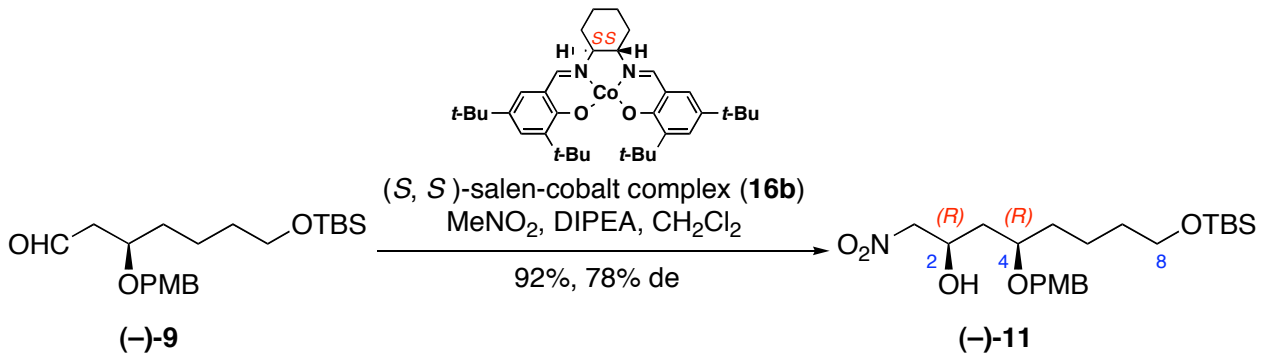

To a solution of aldehyde (-)-9 (243 mg, $0.638 \mathrm{mmol})$ in $\mathrm{CH}_{2} \mathrm{Cl}_{2}(6.40 \mathrm{~mL})$ was added $(S, S)$-salen-cobalt complex $\mathbf{1 6 b}(39.0 \mathrm{mg}, 0.064 \mathrm{mmol})$ and $\mathrm{MeNO}_{2}(1.30 \mathrm{~mL}, 25.52$ mmol) at room temparature. Then the solution was cooled to $-40^{\circ} \mathrm{C}$ and added DIPEA (277 $\mu \mathrm{L}, 1.59 \mathrm{mmol}$ ). After stirring for $69 \mathrm{hr}$, the reaction was quenched with sat. $\mathrm{NH}_{4} \mathrm{Cl}$ aq. (3.0 mL), then the aqueous layer was extracted with $\mathrm{CHCl}_{3}(10 \mathrm{~mL}$ x 3$)$. The combined organic extracts were washed with brine $(10 \mathrm{~mL})$, dried over $\mathrm{Na}_{2} \mathrm{SO}_{4}$, filtered, and evaporated under reduced pressure. Purification by flash chromatography on silica gel (Hexane : EtOAc = 5 : 1) provided the title compound (-)-11 $(258 \mathrm{mg}, 92 \%, 78 \%$ de) as a colorless oil.

The de of desired compound (-)-11 was determined with HPLC (CHIRALCEL OD $4.6 \phi \times 250 \mathrm{~mm}$, Hexane : 2-Propanol = $99: 1,0.9 \mathrm{~mL} / \mathrm{min}, 25{ }^{\circ} \mathrm{C}, 210 \mathrm{~nm}$ )

$\mathbf{R} \boldsymbol{f}=0.45$ (Silica gel, Hexane $:$ EtOAc $=2: 1$ )

$[\alpha]_{\mathbf{D}}{ }^{28}-37.8\left(c 0.63, \mathrm{CHCl}_{3}\right)$

IR $(\mathrm{KBr}) \vee \mathrm{cm}^{-1}$

$3433(\mathrm{OH}), 1612,1514(\mathrm{Ar}-\mathrm{C}), 1554\left(\mathrm{NO}_{2}\right)$

${ }^{1} \mathrm{H}$-NMR (270 MHz, $\mathrm{CDCl}_{3}$ )

$\delta: 7.23\left(\mathrm{~d}, J=8.7 \mathrm{~Hz}, 2 \mathrm{H}, 4-\mathrm{O}-\mathrm{CH}_{2}-\mathrm{Ph}-\mathrm{OCH}_{3}\right)$,

$6.87\left(\mathrm{~d}, J=8.7 \mathrm{~Hz}, 2 \mathrm{H}, 4-\mathrm{O}-\mathrm{CH}_{2}-\mathrm{Ph}-\mathrm{OCH}_{3}\right)$,

$4.59\left(\mathrm{~d}, J=10.9 \mathrm{~Hz}, 1 \mathrm{H}, 4-\mathrm{O}-\mathrm{CH}_{2}-\mathrm{Ph}-\mathrm{OCH}_{3}\right)$,

$4.35\left(\mathrm{~d}, J=10.9 \mathrm{~Hz}, 1 \mathrm{H}, 4-\mathrm{O}-\mathrm{C}_{2}-\mathrm{Ph}-\mathrm{OCH}_{3}\right), 4.39-4.50(\mathrm{~m}, 1 \mathrm{H}, 2-\mathrm{H})$,

4.39-4.29 (m, 2H, 1- $\mathrm{H}_{2}$ ), 3.80 (s, 3H, 4-O- $\left.\mathrm{CH}_{2}-\mathrm{Ph}-\mathrm{OCH}_{3}\right), 3.76-3.69$ (m, 1H, 4-H),

$3.62\left(\mathrm{t}, J=6.1 \mathrm{~Hz}, 2 \mathrm{H}, 8-\mathrm{H}_{2}\right), 1.78-1.69\left(\mathrm{~m}, 2 \mathrm{H}, 3-\mathrm{H}_{2}\right), 1.69-1.63\left(\mathrm{~m}, 2 \mathrm{H}, 5-\mathrm{H}_{2}\right)$,

1.56-1.49 (m, 2H, 7- $\left.\mathrm{H}_{2}\right), 1.45-1.34\left(\mathrm{~m}, 2 \mathrm{H}, 6-\mathrm{H}_{2}\right), 0.90\left(\mathrm{~s}, 9 \mathrm{H}, 8-\mathrm{O}-\mathrm{Si}\left[\left(\mathrm{CH}_{3}\right)_{2}\right] \mathrm{C}\left(\mathrm{C}_{3}\right)_{3}\right)$,

$0.05\left(\mathrm{~s}, 6 \mathrm{H}, 8-\mathrm{O}-\mathrm{Si}\left[\left(\mathrm{C}_{3}\right)_{2}\right] \mathrm{C}\left(\mathrm{CH}_{3}\right)_{3}\right)$

${ }^{13} \mathrm{C}$-NMR (67.5 $\mathrm{MHz}, \mathrm{CDCl}_{3}$ ), major isomer was indicated. 
$\delta$ : 159.3 (1C, 4-O- $\left.\mathrm{CH}_{2}-\underline{\mathrm{Ph}}-\mathrm{OCH}_{3}\right), 129.9$ (1C, 4-O- $\left.\mathrm{CH}_{2}-\underline{\mathrm{Ph}}-\mathrm{OCH}_{3}\right)$, 129.5 (2C, 4-O- $\left.\mathrm{CH}_{2}-\mathrm{Ph}-\mathrm{OCH}_{3}\right), 113.9$ (2C, 4-O- $\left.\mathrm{CH}_{2}-\mathrm{Ph}-\mathrm{OCH}_{3}\right), 80.6(\mathrm{C}-1), 77.8$ (C-4), 70.2 (1C, 4-O- $\mathrm{CH}_{2}-\mathrm{Ph}-\mathrm{OCH}_{3}$ ), 68.1 (C-2), 62.8 (C-8), 55.1 (1C, 4-O-CH 37.4 (C-3), 32.9 (C-5), 32.7 (C-7), 25.9 (3C, 8-O-Si $\left.\left[\left(\mathrm{CH}_{3}\right)_{2}\right] \mathrm{C}\left(\mathrm{CH}_{3}\right)_{3}\right), 20.8$, (C-6), $18.2\left(1 \mathrm{C}, 8-\mathrm{O}-\mathrm{Si}\left[\left(\mathrm{CH}_{3}\right)_{2}\right] \underline{\mathrm{C}}\left(\mathrm{CH}_{3}\right)_{3}\right),-5.4\left(2 \mathrm{C}, 8-\mathrm{O}-\mathrm{Si}\left[\left(\mathrm{CH}_{3}\right)_{2}\right] \mathrm{C}\left(\mathrm{CH}_{3}\right)_{3}\right)$ HR-MS (FAB, NBA matrix)

$\mathbf{m} / \mathbf{z} 464.2431[\mathrm{M}+\mathrm{Na}]^{+}$, Calcd for $\mathrm{C}_{22} \mathrm{H}_{39} \mathrm{NO}_{6} \mathrm{SiNa} 464.2444[\mathrm{M}+\mathrm{Na}]$ 


\section{Scheme 3. Syntheisis of cyclic guanidine (-)-6}

$(2 S, 4 R)-1-[N$ ', $N$ ',-Bis(tert-butoxycarbonyl)guanidino]-8-(tert-butyldimethylsiloxy)-4-( $p$ -methoxybenzyloxy)octan-2-ol (-)-7
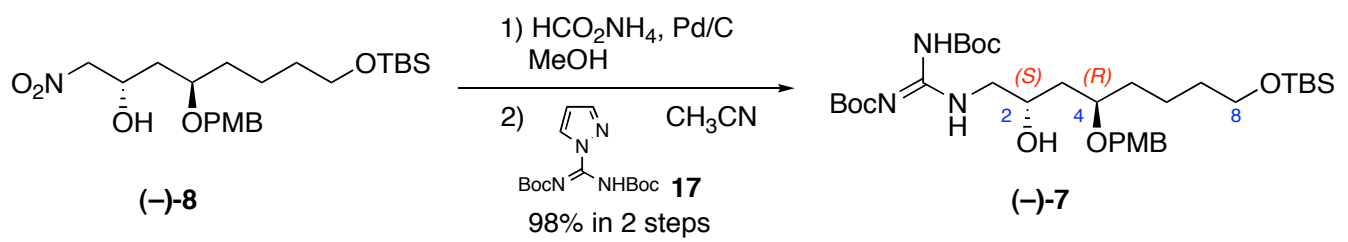

To a solution of alcohol (-)-8 (964 mg, $2.18 \mathrm{mmol})$ in $\mathrm{MeOH}(21.8 \mathrm{~mL})$ was added $10 \% \mathrm{Pd}$ on carbon (232 mg, $0.218 \mathrm{mmol})$, ammonium formate $(619 \mathrm{mg}, 9.82 \mathrm{mmol})$ under $\mathrm{Ar}$ at room temparature. After stirring for $3 \mathrm{hr}$, the reaction mixture was filtered with celite pad, and the pad was washed with $\mathrm{MeOH}$. The filtrate solution was evaporated to remove the solvent, the residue was dissolved in $\mathrm{CHCl}_{3}(30 \mathrm{~mL})$, washed with sat. aq. $\mathrm{NaHCO}_{3}(10 \mathrm{~mL})$, then the organic layer was dried over $\mathrm{Na}_{2} \mathrm{SO}_{4}$, filtered, and evaporated under reduced pressure. The crude product was used to next reaction without further purification. The residue was dissolved in $\mathrm{CH}_{3} \mathrm{CN}(43.7 \mathrm{~mL})$ and then added 1-H-pyrazole-1-( $N, N^{\prime}$-bis(tert-butoxycarbonyl)-carboxamidine 17 (1.02 g, 3.27 $\mathrm{mmol})$. After stirring for $30 \mathrm{~min}$, isopropylamine $(1.88 \mathrm{~mL}, 21.8 \mathrm{mmol})$ was added to the reaction mixture to quench the reaction. The solution was evapolated under reduced pressure to remove the solvent. Purification by flash chromatography on silica gel (Hexane : EtOAc $=5: 1)$ provided the title compound $(-)-7(1.40 \mathrm{~g}, 98 \%$ in 2 steps $)$ as a colorless oil.

$\mathbf{R} \boldsymbol{f}=0.51$ (Silica gel, Hexane $:$ EtOAc $=2: 1$ )

$[\boldsymbol{\alpha}]_{\mathbf{D}}{ }^{22}-7.6\left(c 0.89, \mathrm{CHCl}_{3}\right)$

IR $(\mathrm{KBr}) \vee \mathrm{cm}^{-1}$

$3332(\mathrm{~N}-\mathrm{H}), 1724(\mathrm{C}=\mathrm{O}), 1639(\mathrm{C}=\mathrm{N})$

${ }^{1} \mathrm{H}-\mathrm{NMR}$ (400 MHz, $\mathrm{CDCl}_{3}$ )

$\delta: 11.47\left(\mathrm{~s}, 1 \mathrm{H}, 1-\mathrm{NH}-\mathrm{C}\left[\mathrm{NHCO}-\mathrm{OC}\left(\mathrm{CH}_{3}\right)_{3}\right]\left[\mathrm{NCO}-\mathrm{OC}\left(\mathrm{CH}_{3}\right)_{3}\right]\right)$,

$8.68\left(\mathrm{dd}, J=4.9,6.0 \mathrm{~Hz}, 1 \mathrm{H}, 1-\mathrm{N} \underline{\mathrm{H}}-\mathrm{C}\left[\mathrm{NHCO}-\mathrm{OC}\left(\mathrm{CH}_{3}\right)_{3}\right]\left[\mathrm{NCO}-\mathrm{OC}\left(\mathrm{CH}_{3}\right)_{3}\right]\right)$,

$7.26\left(\mathrm{~d}, J=8.9 \mathrm{~Hz}, 2 \mathrm{H}, 4-\mathrm{O}-\mathrm{CH}_{2}-\mathrm{Ph}-\mathrm{OCH}_{3}\right)$,

$6.86\left(\mathrm{~d}, J=8.7 \mathrm{~Hz}, 2 \mathrm{H}, 4-\mathrm{O}-\mathrm{CH}_{2}-\mathrm{Ph}-\mathrm{OCH}_{3}\right)$,

$4.50\left(\mathrm{~d}, J=11.0 \mathrm{~Hz}, 1 \mathrm{H}, 4-\mathrm{O}-\mathrm{CH}_{2}-\mathrm{Ph}-\mathrm{OCH}_{3}\right)$,

$4.44\left(\mathrm{~d}, J=11.0 \mathrm{~Hz}, 1 \mathrm{H}, 4-\mathrm{O}-\mathrm{CH}_{2}-\mathrm{Ph}-\mathrm{OCH}_{3}\right), 4.06-4.00(\mathrm{~m}, 1 \mathrm{H}, 2-\mathrm{H})$,

3.79 (s, 3H, 4-O- $\left.\mathrm{CH}_{2}-\mathrm{Ph}-\mathrm{OC}_{3}\right), 3.73-3.66(\mathrm{~m}, 1 \mathrm{H}, 4-\mathrm{H}), 3.60\left(\mathrm{t}, J=6.2 \mathrm{~Hz}, 2 \mathrm{H}, 8-\mathrm{H}_{2}\right)$, 
$3.60\left(\mathrm{ddd}, J=3.0,6.0,14.0 \mathrm{~Hz}, 1 \mathrm{H}, 1-\underline{\mathrm{H}}_{2}\right), 3.30\left(\mathrm{ddd}, J=4.9,7.6,14.0 \mathrm{~Hz}, 1 \mathrm{H}, 1-\underline{\mathrm{H}}_{2}\right)$, 1.73 (ddd, $\left.J=3.3,6.9,14.2 \mathrm{~Hz}, 1 \mathrm{H}, 3-\underline{\mathrm{H}}_{2}\right), 1.53$ (ddd, $J=3.3,9.3,14.2 \mathrm{~Hz}, 1 \mathrm{H}, 3-\underline{\mathrm{H}}_{2}$ ), 1.69-1.64 (m, 1H, 5- $\underline{\mathrm{H}}_{2}$ ), 1.57-1.43 (complex m, 1H, 5- $\underline{\mathrm{H}}_{2}$ ), 1.57-1.43 (complex m, 2H, 7- $\mathrm{H}_{2}$ ),

1.49 (s, 9H, 1-NH-C $\left.\left[\mathrm{NHCO}-\mathrm{OC}\left(\mathrm{CH}_{3}\right)_{3}\right]\left[\mathrm{NCO}-\mathrm{OC}\left(\mathrm{C}_{3}\right)_{3}\right]\right)$ $1.47\left(\mathrm{~s}, 9 \mathrm{H}, 1-\mathrm{NH}-\mathrm{C}\left[\mathrm{NHCO}-\mathrm{OC}\left(\mathrm{C}_{\underline{3}}\right)_{3}\right]\left[\mathrm{NCO}-\mathrm{OC}\left(\mathrm{CH}_{3}\right)_{3}\right]\right)$ 1.43-1.31 (m, 2H, 6- $\left.\mathrm{H}_{2}\right), 0.89$ (s, 9H, 8-O-Si[ $\left.\left.\left(\mathrm{CH}_{3}\right)_{2}\right] \mathrm{C}\left(\mathrm{C}_{3}\right)_{3}\right)$, $0.05\left(\mathrm{~s}, 6 \mathrm{H}, 8-\mathrm{O}-\mathrm{Si}\left[\left(\mathrm{C}_{3}\right)_{2}\right] \mathrm{C}\left(\mathrm{CH}_{3}\right)_{3}\right)$

\section{${ }^{13} \mathrm{C}-\mathrm{NMR}\left(67.5 \mathrm{MHz} \mathrm{CDCl}_{3}\right)$}

$\delta$ : $163.1\left(1 \mathrm{C}, 1-\mathrm{NH}-\mathrm{C}\left[\mathrm{NHCO}-\mathrm{OC}\left(\mathrm{CH}_{3}\right)_{3}\right]\left[\mathrm{NCO}-\mathrm{OC}\left(\mathrm{CH}_{3}\right)_{3}\right]\right)$,

159.3 (1C, 4-O- $\left.\mathrm{CH}_{2}-\mathrm{Ph}-\mathrm{OCH}_{3}\right)$,

$156.9\left(1 \mathrm{C}, 1-\mathrm{NH}-\underline{\mathrm{C}}\left[\mathrm{NHCO}-\mathrm{OC}\left(\mathrm{CH}_{3}\right)_{3}\right]\left[\mathrm{NCO}-\mathrm{OC}\left(\mathrm{CH}_{3}\right)_{3}\right]\right)$,

$152.9\left(1 \mathrm{C}, 1-\mathrm{NH}-\mathrm{C}\left[\mathrm{NHCO}-\mathrm{OC}\left(\mathrm{CH}_{3}\right)_{3}\right]\left[\mathrm{NCO}-\mathrm{OC}\left(\mathrm{CH}_{3}\right)_{3}\right]\right)$,

130.4 (1C, 4-O- $\left.\mathrm{CH}_{2}-\mathrm{Ph}-\mathrm{OCH}_{3}\right), 129.4$ (2C, 4-O- $\left.\mathrm{CH}_{2}-\mathrm{Ph}-\mathrm{OCH}_{3}\right)$,

113.7 (2C, 4-O- $\left.\mathrm{CH}_{2}-\underline{\mathrm{Ph}}-\mathrm{OCH}_{3}\right)$,

$83.0\left(1 \mathrm{C}, 1-\mathrm{NH}-\mathrm{C}\left[\mathrm{NHCO}-\mathrm{OC}\left(\mathrm{CH}_{3}\right)_{3}\right]\left[\mathrm{NCO}-\mathrm{O} \underline{\mathrm{C}}\left(\mathrm{CH}_{3}\right)_{3}\right]\right)$,

$79.2\left(1 \mathrm{C}, 1-\mathrm{NH}-\mathrm{C}\left[\mathrm{NHCO}-\mathrm{OC}\left(\mathrm{CH}_{3}\right)_{3}\right]\left[\mathrm{NCO}-\mathrm{OC}\left(\mathrm{CH}_{3}\right)_{3}\right]\right), 76.4(\mathrm{C}-4)$,

71.0 (1C, 3-O- $\left.\underline{\mathrm{CH}}_{2}-\mathrm{Ph}-\mathrm{OCH}_{3}\right), 68.0$ (C-2), 62.9 (C-8), 55.1 (1C, 4-O- $\left.\mathrm{CH}_{2}-\mathrm{Ph}-\mathrm{OCH}_{3}\right)$,

47.3 (C-1), 37.9 (C-3), 33.4 (C-5), 32.8 (C-7),

$28.1\left(3 \mathrm{C}, 1-\mathrm{NH}-\mathrm{C}\left[\mathrm{NHCO}-\mathrm{OC}\left(\mathrm{CH}_{3}\right)_{3}\right]\left[\mathrm{NCO}-\mathrm{OC}\left(\mathrm{CH}_{3}\right)_{3}\right]\right)$

27.9 (3C, 1-NH-C $\left.\left[\mathrm{NHCO}-\mathrm{OC}\left(\mathrm{CH}_{3}\right)_{3}\right]\left[\mathrm{NCO}-\mathrm{OC}\left(\mathrm{CH}_{3}\right)_{3}\right]\right)$

25.9 (3C, 8-O-Si $\left.\left[\left(\mathrm{CH}_{3}\right)_{2}\right] \mathrm{C}\left(\mathrm{CH}_{3}\right)_{3}\right), 21.5$, (C-6),

$18.2\left(1 \mathrm{C}, 8-\mathrm{O}-\mathrm{Si}\left[\left(\mathrm{CH}_{3}\right)_{2}\right] \underline{\mathrm{C}}\left(\mathrm{CH}_{3}\right)_{3}\right),-5.4\left(2 \mathrm{C}, 8-\mathrm{O}-\mathrm{Si}\left[\left(\mathrm{CH}_{3}\right)_{2}\right] \mathrm{C}\left(\mathrm{CH}_{3}\right)_{3}\right)$

\section{HR-MS (FAB, NBA matrix)}

$\mathbf{m} / \mathbf{z} 654.4164[\mathrm{M}+\mathrm{H}]^{+}$, Calcd for $\mathrm{C}_{33} \mathrm{H}_{60} \mathrm{~N}_{3} \mathrm{O}_{8} \mathrm{Si} 654.4150[\mathrm{M}+\mathrm{H}]$ 


\section{Determination of the stereochemistry of nitroaldol product (-)-8.}

- $\quad(2 S, 4 R)-N, N^{\prime}$-Bis(tert-butoxycarbonyl)-8-(tert-butyldimethylsiloxy)-4(p-methoxybenzyloxy)-2-[(S)- $\alpha$-methoxy- $\alpha$-trifluoromethylphenylacetoxy] guanidine (+)-II

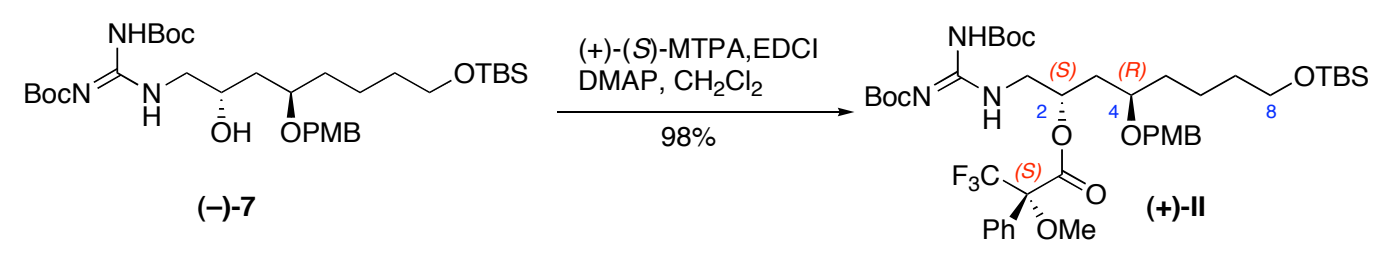

Under Ar atmosphere, a solution of $(2 S, 4 R)$-guanidine alcohol (-)-7 (32.9 g, 50.5 $\mu \mathrm{mol})$ in $\mathrm{CH}_{2} \mathrm{Cl}_{2}(1.00 \mathrm{~mL})$ was added $(+)-(S)$-MTPA $(35.4 \mathrm{mg}, 0.151 \mathrm{mmol})$, EDCI (28.9 mg, $0.151 \mathrm{mmol}$ ) and DMAP $(0.6 \mathrm{mg}, 5.05 \mu \mathrm{mol})$. After stirring for $2.5 \mathrm{hr}$, the reaction mixture was quenched with $\mathrm{H}_{2} \mathrm{O}(1.0 \mathrm{~mL})$. Then the organic layer was separated and the aqueous layer was extracted with $\mathrm{CH}_{2} \mathrm{Cl}_{2}(5.0 \mathrm{~mL} \mathrm{X} \mathrm{3)}$. The combined organic extracts were washed with brine $(5.0 \mathrm{~mL})$, dried, filtered and concentrated. Purification by preparative TLC (Hexane : EtOAc $=2: 1$ ) gave the title compound (+)-II (43.0 mg, 98\%) as a colorless oil.

$\mathbf{R} \boldsymbol{f}=0.57$ (Silica gel, Hexane $:$ EtOAc $=2: 1$ )

$[\alpha]_{\mathrm{D}}{ }^{26}+1.44\left(c\right.$ 2.06, $\left.\mathrm{CHCl}_{3}\right)$

IR $(\mathrm{NaCl}) v \mathrm{~cm}^{-1}$

$3332(\mathrm{~N}-\mathrm{H}), 1749,1722(\mathrm{C}=\mathrm{O}), 1641(\mathrm{C}=\mathrm{N})$

\section{${ }^{1} \mathrm{H}-\mathrm{NMR}\left(400 \mathrm{MHz} \mathrm{CDCl}_{3}\right.$ )}

$\delta: 11.41\left(\mathrm{~s}, 1 \mathrm{H}, 1-\mathrm{NH}-\mathrm{C}\left[\mathrm{NHCO}-\mathrm{OC}\left(\mathrm{CH}_{3}\right)_{3}\right]\left[\mathrm{NCO}-\mathrm{OC}\left(\mathrm{CH}_{3}\right)_{3}\right]\right)$,

$8.43\left(\mathrm{t}, J=5.2 \mathrm{~Hz}, 1 \mathrm{H}, 1-\mathrm{N} \underline{\mathrm{H}}-\mathrm{C}\left[\mathrm{NHCO}-\mathrm{OC}\left(\mathrm{CH}_{3}\right)_{3}\right]\left[\mathrm{NCO}-\mathrm{OC}\left(\mathrm{CH}_{3}\right)_{3}\right]\right)$,

7.54-7.52 (m, 2H, 2-OC(O)C( $\left.\left(\mathrm{CF}_{3}\right)\left(\mathrm{OCH}_{3}\right) \underline{\mathrm{Ph}}\right)$,

7.42-7.36 (m, 2H, 2-OC(O) $\left.\left(\mathrm{CF}_{3}\right)\left(\mathrm{OCH}_{3}\right) \underline{\mathrm{Ph}}\right)$,

$7.27\left(\mathrm{~d}, J=8.6 \mathrm{~Hz}, 2 \mathrm{H}, 4-\mathrm{O}-\mathrm{CH}_{2}-\mathrm{Ph}-\mathrm{OCH}_{3}\right)$,

$6.86\left(\mathrm{~d}, J=8.6 \mathrm{~Hz}, 2 \mathrm{H}, 4-\mathrm{O}-\mathrm{CH}_{2}-\mathrm{Ph}-\mathrm{OCH}_{3}\right)$,

5.55-5.49 (m, 1H, 2-H), 4.43 (d, $J=11.0 \mathrm{~Hz}, 1 \mathrm{H}, 4-\mathrm{O}-\mathrm{C}_{2}-\mathrm{Ph}-\mathrm{OCH}_{3}$ ),

4.32 (d, $\left.J=11.0 \mathrm{~Hz}, 1 \mathrm{H}, 4-\mathrm{O}-\mathrm{C}_{2}-\mathrm{Ph}-\mathrm{OCH}_{3}\right), 3.79$ (s, 3H, 4-O-CH$-\mathrm{Ph}-\mathrm{OC}_{2}{ }_{3}$ ),

3.74-3.72 (m, 1H, 1- $\left.\underline{\mathrm{H}}_{2}\right), 3.60,\left(\mathrm{t}, J=6.3 \mathrm{~Hz}, 2 \mathrm{H}, 8-\mathrm{H}_{2}\right), 3.57-3.55\left(\mathrm{~m}, 1 \mathrm{H}, 1-\underline{\mathrm{H}}_{2}\right)$,

$3.49\left(\mathrm{~m}, 3 \mathrm{H}, 2-\mathrm{OC}(\mathrm{O}) \mathrm{C}\left(\mathrm{CF}_{3}\right)\left(\mathrm{OC}_{3}\right) \mathrm{Ph}\right), 3.44-3.38(\mathrm{~m}, 1 \mathrm{H}, 4-\mathrm{H})$,

1.82-1.78 (m, 2H, 3- $\left.\mathrm{H}_{2}\right), 1.66-1.50\left(\mathrm{~m}, 2 \mathrm{H}, 5-\mathrm{H}_{2}\right), 1.53-1.48\left(\mathrm{~m}, 2 \mathrm{H}, 7-\mathrm{H}_{2}\right)$,

$1.47\left(\mathrm{~s}, 9 \mathrm{H}, 1-\mathrm{NH}-\mathrm{C}\left[\mathrm{NHCO}-\mathrm{OC}\left(\mathrm{CH}_{3}\right)_{3}\right]\left[\mathrm{NCO}-\mathrm{OC}\left(\mathrm{C}_{\underline{3}}\right)_{3}\right]\right)$ 
$1.46\left(\mathrm{~s}, 9 \mathrm{H}, 1-\mathrm{NH}-\mathrm{C}\left[\mathrm{NHCO}-\mathrm{OC}\left(\mathrm{C}_{3}\right)_{3}\right]\left[\mathrm{NCO}-\mathrm{OC}\left(\mathrm{CH}_{3}\right)_{3}\right]\right)$

1.39-1.30 (m, 2H, 6- $\left.\mathrm{H}_{2}\right), 0.89$ (s, 9H, 8-O-Si[ $\left.\left.\left(\mathrm{CH}_{3}\right)_{2}\right] \mathrm{C}\left(\mathrm{C}_{2}\right)_{3}\right)$,

0.05 (s, 6H, 8-O-Si[ $\left.\left.\left(\mathrm{C}_{3}\right)_{2}\right] \mathrm{C}\left(\mathrm{CH}_{3}\right)_{3}\right)$

${ }^{13} \mathrm{C}-\mathrm{NMR}\left(67.5 \mathrm{MHz}, \mathrm{CDCl}_{3}\right)$

$\delta: 166.2\left(1 \mathrm{C}, 2-\mathrm{OC}(\mathrm{O}) \mathrm{C}\left(\mathrm{CF}_{3}\right)\left(\mathrm{OCH}_{3}\right) \mathrm{Ph}\right)$,

$163.3\left(1 \mathrm{C}, 1-\mathrm{NH}-\mathrm{C}\left[\mathrm{NHCO}-\mathrm{OC}\left(\mathrm{CH}_{3}\right)_{3}\right]\left[\mathrm{NCO}-\mathrm{OC}\left(\mathrm{CH}_{3}\right)_{3}\right]\right)$,

$159.1\left(1 \mathrm{C}, 4-\mathrm{O}-\mathrm{CH}_{2}-\mathrm{Ph}-\mathrm{OCH}_{3}\right)$,

$156.4\left(1 \mathrm{C}, 1-\mathrm{NH}-\underline{\mathrm{C}}\left[\mathrm{NHCO}-\mathrm{OC}\left(\mathrm{CH}_{3}\right)_{3}\right]\left[\mathrm{NCO}-\mathrm{OC}\left(\mathrm{CH}_{3}\right)_{3}\right]\right)$,

$152.8\left(1 \mathrm{C}, 1-\mathrm{NH}-\mathrm{C}\left[\mathrm{NHCO}-\mathrm{OC}\left(\mathrm{CH}_{3}\right)_{3}\right]\left[\mathrm{NCO}-\mathrm{OC}\left(\mathrm{CH}_{3}\right)_{3}\right]\right)$,

$131.9\left(1 \mathrm{C}, 2-\mathrm{OC}(\mathrm{O}) \mathrm{C}\left(\mathrm{CF}_{3}\right)\left(\mathrm{OCH}_{3}\right) \mathrm{Ph}\right), 130.4\left(1 \mathrm{C}, 4-\mathrm{O}-\mathrm{CH}_{2}-\mathrm{Ph}-\mathrm{OCH}_{3}\right)$,

$129.6\left(2 \mathrm{C}, 2-\mathrm{OC}(\mathrm{O}) \mathrm{C}\left(\mathrm{CF}_{3}\right)\left(\mathrm{OCH}_{3}\right) \underline{\mathrm{Ph}}\right), 129.5\left(2 \mathrm{C}, 4-\mathrm{O}-\mathrm{CH}_{2}-\mathrm{Ph}-\mathrm{OCH}_{3}\right)$,

$128.4\left(3 \mathrm{C}, 2-\mathrm{OC}(\mathrm{O}) \mathrm{C}_{(}\left(\mathrm{CF}_{3}\right)\left(\mathrm{OCH}_{3}\right) \underline{\mathrm{Ph}}\right), 127.3\left(1 \mathrm{C}, 2-\mathrm{OC}(\mathrm{O}) \mathrm{C}\left(\mathrm{CF}_{3}\right)\left(\mathrm{OCH}_{3}\right) \underline{\mathrm{Ph}}\right)$,

$113.8\left(2 \mathrm{C}, 4-\mathrm{O}-\mathrm{CH}_{2}-\mathrm{Ph}-\mathrm{OCH}_{3}\right), 84.4\left(1 \mathrm{C}, 2-\mathrm{OC}(\mathrm{O}) \underline{\mathrm{C}}\left(\mathrm{CF}_{3}\right)\left(\mathrm{OCH}_{3}\right) \mathrm{Ph}\right)$,

$83.1\left(1 \mathrm{C}, 1-\mathrm{NH}-\mathrm{C}\left[\mathrm{NHCO}-\mathrm{OC}\left(\mathrm{CH}_{3}\right)_{3}\right]\left[\mathrm{NCO}-\mathrm{OC}\left(\mathrm{CH}_{3}\right)_{3}\right]\right)$,

$79.2\left(1 \mathrm{C}, 1-\mathrm{NH}-\mathrm{C}\left[\mathrm{NHCO}-\mathrm{OC}\left(\mathrm{CH}_{3}\right)_{3}\right]\left[\mathrm{NCO}-\mathrm{O} \underline{\mathrm{C}}\left(\mathrm{CH}_{3}\right)_{3}\right]\right), 74.8(\mathrm{C}-4), 73.0(\mathrm{C}-2)$,

$70.8\left(1 \mathrm{C}, 4-\mathrm{O}-\mathrm{CH}_{2}-\mathrm{Ph}-\mathrm{OCH}_{3}\right), 62.9(\mathrm{C}-8), 55.4\left(1 \mathrm{C}, 2-\mathrm{OC}(\mathrm{O}) \mathrm{C}\left(\mathrm{CF}_{3}\right)\left(\mathrm{OCH}_{3}\right) \mathrm{Ph}\right)$,

55.2 (1C, 4-O- $\mathrm{CH}_{2}-\mathrm{Ph}-\mathrm{OCH}_{3}$ ), 44.1 (C-1), 37.0 (C-3), 33.4 (C-5), 32.9 (C-7),

28.2 (3C, 1-NH-C $\left.\left[\mathrm{NHCO}-\mathrm{OC}\left(\mathrm{CH}_{3}\right)_{3}\right]\left[\mathrm{NCO}-\mathrm{OC}\left(\mathrm{CH}_{3}\right)_{3}\right]\right)$

27.9 (3C, 1-NH-C $\left.\left[\mathrm{NHCO}-\mathrm{OC}\left(\mathrm{CH}_{3}\right)_{3}\right]\left[\mathrm{NCO}-\mathrm{OC}\left(\mathrm{CH}_{3}\right)_{3}\right]\right)$

25.9 (3C, 8-O-Si $\left.\left[\left(\mathrm{CH}_{3}\right)_{2}\right] \mathrm{C}\left(\mathrm{CH}_{3}\right)_{3}\right), 21.0$, (C-6),

$18.3\left(1 \mathrm{C}, 8-\mathrm{O}-\mathrm{Si}\left[\left(\mathrm{CH}_{3}\right)_{2}\right] \mathrm{C}\left(\mathrm{CH}_{3}\right)_{3}\right),-5.3\left(2 \mathrm{C}, 8-\mathrm{O}-\mathrm{Si}\left[\left(\mathrm{CH}_{3}\right)_{2}\right] \mathrm{C}\left(\mathrm{CH}_{3}\right)_{3}\right)$

HR-MS (FAB, NBA matrix)

$\mathbf{m} / \mathbf{z} 870.4555[\mathrm{M}+\mathrm{H}]^{+}$, Calcd for $\mathrm{C}_{43} \mathrm{H}_{67} \mathrm{~N}_{3} \mathrm{O}_{10} \mathrm{Si} 870.4548[\mathrm{M}+\mathrm{H}]$ 
- $\quad(2 S, 4 R)-N, N^{\prime}$-Bis(tert-butoxycarbonyl)-8-(tert-butyldimethylsiloxy)-4(p-methoxybenzyloxy)-2-[(R)- $\alpha$-methoxy- $\alpha$-trifluoromethylphenylacetoxy]guanidine (-)-III

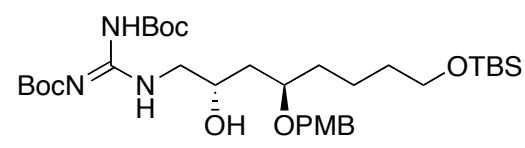

$(-)-7$

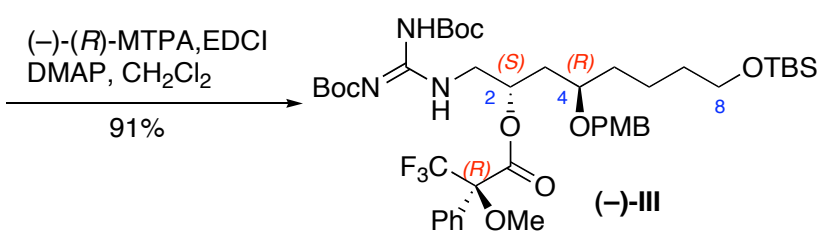

Under Ar atmosphere, a solution of (2S, 4R)-guanidine alcohol (-)-21 (33.5 mg, 51.4 $\mu \mathrm{mol})$ in $\mathrm{CH}_{2} \mathrm{Cl}_{2}(1.00 \mathrm{~mL})$ was added $(-)-(R)$-MTPA (36.1 mg, $\left.0.154 \mathrm{mmol}\right)$, EDCI $(29.5 \mathrm{mg}, 0.154 \mathrm{mmol})$ and DMAP $(0.6 \mathrm{mg}, 5.14 \mu \mathrm{mol})$. After stirring for $6 \mathrm{hr}$, the reaction mixture was quenched with $\mathrm{H}_{2} \mathrm{O}(1.0 \mathrm{~mL})$. Then the organic layer was separated and the aqueous layer was extracted with $\mathrm{CH}_{2} \mathrm{Cl}_{2}(5.0 \mathrm{~mL} \mathrm{X} \mathrm{3)}$. The combined organic extracts were washed with brine $(5.0 \mathrm{~mL})$, dried, filtered and concentrated. Purification by preparative TLC (Hexane : EtOAc $=2: 1$ ) gave the title compound (-)-III (40.8 mg, 91\%) as a colorless oil.

$\mathbf{R} \boldsymbol{f}=0.59$ (Silica gel, Hexane $:$ EtOAc $=2: 1$ )

$[\alpha]_{\mathbf{D}}{ }^{27}-35.5\left(c 1.98, \mathrm{CHCl}_{3}\right)$

IR $(\mathrm{NaCl}) \vee \mathrm{cm}^{-1}$

$3332(\mathrm{~N}-\mathrm{H}), 1749,1724(\mathrm{C}=\mathrm{O}), 1641(\mathrm{C}=\mathrm{N})$

${ }^{1} \mathrm{H}-\mathrm{NMR}$ (400 MHz, $\mathrm{CDCl}_{3}$ )

$\delta: 11.43\left(\mathrm{~s}, 1 \mathrm{H}, 1-\mathrm{NH}-\mathrm{C}\left[\mathrm{NHCO}-\mathrm{OC}\left(\mathrm{CH}_{3}\right)_{3}\right]\left[\mathrm{NCO}-\mathrm{OC}\left(\mathrm{CH}_{3}\right)_{3}\right]\right)$,

$8.54\left(\mathrm{t}, 1 \mathrm{H}, J=5.2 \mathrm{~Hz}, 1-\mathrm{NH}-\mathrm{C}\left[\mathrm{NHCO}-\mathrm{OC}\left(\mathrm{CH}_{3}\right)_{3}\right]\left[\mathrm{NCO}-\mathrm{OC}\left(\mathrm{CH}_{3}\right)_{3}\right]\right)$,

7.55-7.51 (m, 2H, 2-OC(O) $\left.\left(\mathrm{CF}_{3}\right)\left(\mathrm{OCH}_{3}\right) \underline{\mathrm{Ph}}\right)$,

7.42-7.33 (m, 3H, 2-OC(O) $\left.\left(\mathrm{CF}_{3}\right)\left(\mathrm{OCH}_{3}\right) \underline{\mathrm{Ph}}\right)$,

$7.28\left(\mathrm{~d}, J=8.6 \mathrm{~Hz}, 2 \mathrm{H}, 4-\mathrm{O}-\mathrm{CH}_{2}-\mathrm{Ph}-\mathrm{OCH}_{3}\right)$,

$6.88\left(\mathrm{~d}, J=8.6 \mathrm{~Hz}, 2 \mathrm{H}, 4-\mathrm{O}-\mathrm{CH}_{2}-\underline{\mathrm{Ph}}-\mathrm{OCH}_{3}\right)$,

5.56-5.51 (m, 1H, 2-H), 4.39 (d, $\left.J=10.5 \mathrm{~Hz}, 1 \mathrm{H}, 4-\mathrm{O}-\mathrm{C}_{2}-\mathrm{Ph}-\mathrm{OCH}_{3}\right)$,

4.27 (d, $\left.J=10.5 \mathrm{~Hz}, 1 \mathrm{H}, 4-\mathrm{O}-\mathrm{C}_{2}-\mathrm{Ph}-\mathrm{OCH}_{3}\right), 3.80$ (s, 3H, 4-O- $\left.\mathrm{CH}_{2}-\mathrm{Ph}-\mathrm{OC}_{3}\right)$,

3.76-3.74 (m, 1H, 1- $\left.\underline{\mathrm{H}}_{2}\right), 3.57,\left(\mathrm{t}, J=6.3 \mathrm{~Hz}, 2 \mathrm{H}, 8-\mathrm{H}_{2}\right), 3.57-3.55\left(\mathrm{~m}, 1 \mathrm{H}, 1-\underline{\mathrm{H}}_{2}\right)$,

$3.55\left(\mathrm{~m}, 3 \mathrm{H}, 2-\mathrm{OC}(\mathrm{O}) \mathrm{C}\left(\mathrm{CF}_{3}\right)\left(\mathrm{OC}_{3}\right) \mathrm{Ph}\right), 3.27-3.21(\mathrm{~m}, 1 \mathrm{H}, 4-\mathrm{H})$,

1.75-1.70 (m, 2H, 3- $\mathrm{H}_{2}$ ), 1.59-1.50 (complex m, 2H, 5- $\mathrm{H}_{2}$ ),

1.59-1.50 (complex m, 2H, 7- $\mathrm{H}_{2}$ ),

$1.47\left(\mathrm{~s}, 9 \mathrm{H}, 1-\mathrm{NH}-\mathrm{C}\left[\mathrm{NHCO}-\mathrm{OC}\left(\mathrm{CH}_{3}\right)_{3}\right]\left[\mathrm{NCO}-\mathrm{OC}\left(\mathrm{C}_{3}\right)_{3}\right]\right)$ 
1.47 (s, 9H, 1-NH-C $\left.\left[\mathrm{NHCO}-\mathrm{OC}\left(\mathrm{CH}_{3}\right)_{3}\right]\left[\mathrm{NCO}-\mathrm{OC}\left(\mathrm{CH}_{3}\right)_{3}\right]\right)$

1.33-1.21 (m, 2H, 6- $\left.\mathrm{H}_{2}\right), 0.89$ (s, 9H, 8-O-Si[ $\left.\left.\left(\mathrm{CH}_{3}\right)_{2}\right] \mathrm{C}\left(\mathrm{C}_{2}\right)_{3}\right)$,

0.04 (s, 6H, 8-O-Si[ $\left.\left.\left(\mathrm{C}_{3}\right)_{2}\right] \mathrm{C}\left(\mathrm{CH}_{3}\right)_{3}\right)$

${ }^{13} \mathrm{C}-\mathrm{NMR}\left(67.5 \mathrm{MHz}, \mathrm{CDCl}_{3}\right)$

$\delta: 166.3\left(1 \mathrm{C}, 2-\mathrm{OC}(\mathrm{O}) \mathrm{C}\left(\mathrm{CF}_{3}\right)\left(\mathrm{OCH}_{3}\right) \mathrm{Ph}\right)$,

$163.4\left(1 \mathrm{C}, 1-\mathrm{NH}-\mathrm{C}\left[\mathrm{NHCO}-\mathrm{OC}\left(\mathrm{CH}_{3}\right)_{3}\right]\left[\mathrm{NCO}-\mathrm{OC}\left(\mathrm{CH}_{3}\right)_{3}\right]\right)$,

$159.2\left(1 \mathrm{C}, 4-\mathrm{O}-\mathrm{CH}_{2}-\mathrm{Ph}-\mathrm{OCH}_{3}\right)$,

$156.4\left(1 \mathrm{C}, 1-\mathrm{NH}-\underline{\mathrm{C}}\left[\mathrm{NHCO}-\mathrm{OC}\left(\mathrm{CH}_{3}\right)_{3}\right]\left[\mathrm{NCO}-\mathrm{OC}\left(\mathrm{CH}_{3}\right)_{3}\right]\right)$,

$152.9\left(1 \mathrm{C}, 1-\mathrm{NH}-\mathrm{C}\left[\mathrm{NHCO}-\mathrm{OC}\left(\mathrm{CH}_{3}\right)_{3}\right]\left[\mathrm{NCO}-\mathrm{OC}\left(\mathrm{CH}_{3}\right)_{3}\right]\right)$,

$132.1\left(1 \mathrm{C}, 2-\mathrm{OC}(\mathrm{O}) \mathrm{C}\left(\mathrm{CF}_{3}\right)\left(\mathrm{OCH}_{3}\right) \mathrm{Ph}\right), 130.5\left(1 \mathrm{C}, 4-\mathrm{O}-\mathrm{CH}_{2}-\mathrm{Ph}-\mathrm{OCH}_{3}\right)$,

$129.5\left(2 \mathrm{C}, 2-\mathrm{OC}(\mathrm{O}) \mathrm{C}\left(\mathrm{CF}_{3}\right)\left(\mathrm{OCH}_{3}\right) \underline{\mathrm{Ph}}\right), 129.5\left(2 \mathrm{C}, 4-\mathrm{O}-\mathrm{CH}_{2}-\mathrm{Ph}-\mathrm{OCH}_{3}\right)$,

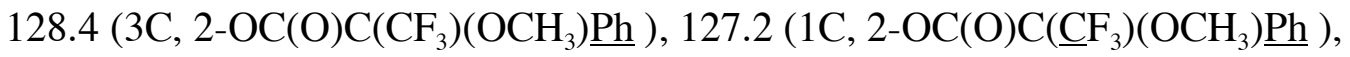

$113.8\left(2 \mathrm{C}, 4-\mathrm{O}-\mathrm{CH}_{2}-\mathrm{Ph}-\mathrm{OCH}_{3}\right), 84.2\left(1 \mathrm{C}, 2-\mathrm{OC}(\mathrm{O}) \underline{\mathrm{C}}\left(\mathrm{CF}_{3}\right)\left(\mathrm{OCH}_{3}\right) \mathrm{Ph}\right)$,

$83.2\left(1 \mathrm{C}, 1-\mathrm{NH}-\mathrm{C}\left[\mathrm{NHCO}-\mathrm{OC}\left(\mathrm{CH}_{3}\right)_{3}\right]\left[\mathrm{NCO}-\mathrm{OC}\left(\mathrm{CH}_{3}\right)_{3}\right]\right)$,

$79.2\left(1 \mathrm{C}, 1-\mathrm{NH}-\mathrm{C}\left[\mathrm{NHCO}-\mathrm{OC}\left(\mathrm{CH}_{3}\right)_{3}\right]\left[\mathrm{NCO}-\mathrm{O} \underline{\mathrm{C}}\left(\mathrm{CH}_{3}\right)_{3}\right]\right), 74.9(\mathrm{C}-4), 72.8(\mathrm{C}-2)$,

$70.9\left(1 \mathrm{C}, 4-\mathrm{O}-\mathrm{CH}_{2}-\mathrm{Ph}-\mathrm{OCH}_{3}\right), 62.9(\mathrm{C}-8), 55.5\left(1 \mathrm{C}, 2-\mathrm{OC}(\mathrm{O}) \mathrm{C}\left(\mathrm{CF}_{3}\right)\left(\mathrm{OCH}_{3}\right) \mathrm{Ph}\right)$,

55.2 (1C, 4-O- $\left.\mathrm{CH}_{2}-\mathrm{Ph}-\mathrm{OCH}_{3}\right), 44.3$ (C-1), 37.0 (C-3), 33.4 (C-5), 32.8 (C-7),

28.2 (3C, 1-NH-C $\left.\left[\mathrm{NHCO}-\mathrm{OC}\left(\mathrm{CH}_{3}\right)_{3}\right]\left[\mathrm{NCO}-\mathrm{OC}\left(\mathrm{CH}_{3}\right)_{3}\right]\right)$

27.9 (3C, 1-NH-C $\left.\left[\mathrm{NHCO}-\mathrm{OC}\left(\mathrm{CH}_{3}\right)_{3}\right]\left[\mathrm{NCO}-\mathrm{OC}\left(\mathrm{CH}_{3}\right)_{3}\right]\right)$

25.9 (3C, 8-O-Si $\left.\left[\left(\mathrm{CH}_{3}\right)_{2}\right] \mathrm{C}\left(\mathrm{CH}_{3}\right)_{3}\right), 21.0$, (C-6),

$18.3\left(1 \mathrm{C}, 8-\mathrm{O}-\mathrm{Si}\left[\left(\mathrm{CH}_{3}\right)_{2}\right] \mathrm{C}\left(\mathrm{CH}_{3}\right)_{3}\right),-5.3\left(2 \mathrm{C}, 8-\mathrm{O}-\mathrm{Si}\left[\left(\mathrm{CH}_{3}\right)_{2}\right] \mathrm{C}\left(\mathrm{CH}_{3}\right)_{3}\right)$

HR-MS (FAB, NBA matrix)

$\mathbf{m} / \mathbf{z} 870.4568[\mathrm{M}+\mathrm{H}]^{+}$, Calcd for $\mathrm{C}_{43} \mathrm{H}_{67} \mathrm{~N}_{3} \mathrm{O}_{10} \mathrm{Si} 870.4548[\mathrm{M}+\mathrm{H}]$

Analysis on chemical shifts of Mosher's ester (+)-II and (-)-III.

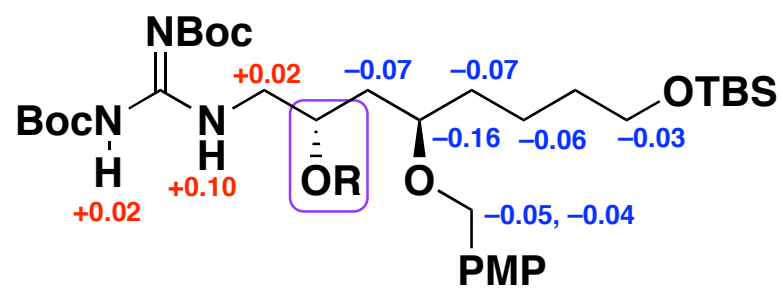

$$
\begin{array}{ll}
\left(\Delta \delta=\delta_{(S)}-\delta_{(R)}\right) & (+)-\text {-II : } R=(S)-(-)-M T P A \\
(-)-I I I: R=(R)-(+)-M T P A
\end{array}
$$




\section{Scheme 3. Synthesis of cyclic guanidine (-)-6}

- $\quad\left(5 R, 4^{\prime} R\right)-6-[N, 3$-Bis (tert-butoxycarbonyl)-2'-iminoimidazolidin-4'-yl]-5-( $p$-methox ybenzyloxy)hexyloxy-tert-butyldimethylsilane (-)-6
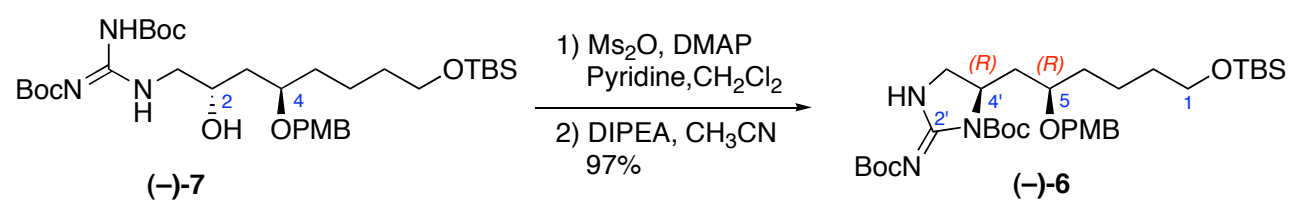

To a solution of $(2 \mathrm{~S}, 4 R)$-guanidine alcohol (-)-7 $(86.0 \mathrm{mg}, 0.131 \mathrm{mmol})$ in $\mathrm{CH}_{2} \mathrm{Cl}_{2}$ $(1.31 \mathrm{~mL})$ was added pyridine $(106 \mathrm{~mL}, 1.31 \mathrm{mmol})$, methanesulfonic anhydride $(34.0$ $\mathrm{mg}, 0.197 \mathrm{mmol})$, DMAP $(0.80 \mathrm{mg}, 6.56 \mu \mathrm{mol})$ at rooom temparature under Ar. After stirring for $15 \mathrm{~min}$, the reaction mixture was quenched with sat. $\mathrm{NH}_{4} \mathrm{Cl}$ aq. $(1.0 \mathrm{~mL})$. Then the organic layer was separated, and the aqueous layer was extracted with $\mathrm{CHCl}_{3}$ (10 mL X 3). The combined organic extracts were washed with brine $(5.0 \mathrm{~mL})$, dried over $\mathrm{Na}_{2} \mathrm{SO}_{4}$, filtered, and evaporated under reduced pressure. Purification by flash chromatography on silica gel (Hexane : EtOAc $=4: 1$ ) provided the title compound (-)-6 (81 $\mathrm{mg}, 97 \%)$ as a pale yellow oil.

$\mathbf{R} \boldsymbol{f}=0.35$ (Silica gel, Hexane $:$ EtOAc $=1: 1$ )

$[\alpha]_{\mathbf{D}}{ }^{25}-32.8\left(c 0.63, \mathrm{CHCl}_{3}\right)$

IR $(\mathrm{KBr}) \vee \mathrm{cm}^{-1}$

$1760(\mathrm{C}=\mathrm{O}), 1647(\mathrm{C}=\mathrm{N})$

${ }^{1} \mathrm{H}-\mathrm{NMR}\left(\mathbf{2 7 0} \mathrm{MHz}, \mathrm{CDCl}_{3}\right)$

$\delta: 7.22\left(\mathrm{~d}, J=8.6 \mathrm{~Hz}, 2 \mathrm{H}, 5-\mathrm{O}-\mathrm{CH}_{2}-\mathrm{Ph}-\mathrm{OCH}_{3}\right)$,

$6.86\left(\mathrm{~d}, J=8.6 \mathrm{~Hz}, 2 \mathrm{H}, 5-\mathrm{O}-\mathrm{CH}_{2}-\mathrm{Ph}-\mathrm{OCH}_{3}\right)$,

$4.50\left(\mathrm{~d}, J=11.2 \mathrm{~Hz}, 1 \mathrm{H}, 5-\mathrm{O}-\mathrm{CH}_{2}-\mathrm{Ph}-\mathrm{OCH}_{3}\right)$,

$4.28\left(\mathrm{~d}, J=11.2 \mathrm{~Hz}, 1 \mathrm{H}, 5-\mathrm{O}-\mathrm{C}_{2}-\mathrm{Ph}-\mathrm{OCH}_{3}\right), 4.30-4.26$ (m, $1 \mathrm{H}, 4$ '-H),

3.80 (s, $\left.3 \mathrm{H}, 5-\mathrm{O}-\mathrm{CH}_{2}-\mathrm{Ph}-\mathrm{OC}_{\underline{3}}\right), 3.60$ (t, $J=6.3 \mathrm{~Hz}, 2 \mathrm{H}, 1-\mathrm{H}_{2}$ ),

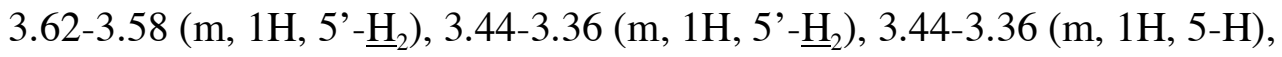

$1.92\left(\mathrm{ddd}, J=2.6,9.2,13.4 \mathrm{~Hz}, 1 \mathrm{H}, 6-\underline{\mathrm{H}}_{2}\right), 1.73-1.62\left(\mathrm{~m}, 1 \mathrm{H}, 6-\underline{\mathrm{H}}_{2}\right)$

1.62-1.55 (m, 2H, 4- $\mathrm{H}_{2}$ ), 1.55-1.33 (complex m, 2H, 3- $\mathrm{H}_{2}$ ),

1.55-1.33 (complex m, 2H, 2- $\left.\mathrm{H}_{2}\right), 1.50$ (s, 9H, 2'-NCO-OC( $\left.\left(\underline{\mathrm{C}}_{3}\right)_{3}\right)$,

1.48 (s, 9H, 3'-CO-OC( $\left.\left.\underline{\mathrm{CH}}_{3}\right)_{3}\right), 0.89$ (s, 9H, 1-O-Si $\left.\left[\left(\mathrm{CH}_{3}\right)_{2}\right] \mathrm{C}\left(\mathrm{C}_{3}\right)_{3}\right)$,

0.05 (s, 6H, 1-O-Si $\left.\left[\left(\mathrm{C}_{3}\right)_{2}\right] \mathrm{C}\left(\mathrm{CH}_{3}\right)_{3}\right)$

${ }^{13} \mathrm{C}-\mathrm{NMR}\left(67.5 \mathrm{MHz} \mathrm{CDCl}_{3}\right.$ ) 
$\delta$ : $159.0\left(1 \mathrm{C}, 5-\mathrm{O}-\mathrm{CH}_{2}-\underline{\mathrm{Ph}}-\mathrm{OCH}_{3}\right), 130.4$ (1C, 5-O- $\left.\mathrm{CH}_{2}-\underline{\mathrm{Ph}}-\mathrm{OCH}_{3}\right)$, $129.2\left(2 \mathrm{C}, 5-\mathrm{O}-\mathrm{CH}_{2}-\mathrm{Ph}-\mathrm{OCH}_{3}\right), 113.5$ (2C, 5-O- $\left.\mathrm{CH}_{2}-\mathrm{Ph}-\mathrm{OCH}_{3}\right)$, 82.7 (1C, 2'-NCO-OC $\left.\left(\mathrm{CH}_{3}\right)_{3}\right), 80.3\left(1 \mathrm{C}, 3^{\prime}\right.$ - $\left.\mathrm{CO}-\mathrm{O} \underline{\mathrm{C}}\left(\mathrm{CH}_{3}\right)_{3}\right)$

74.4 (C-5), 70.2 (1C, 5-O- $\left.\mathrm{CH}_{2}-\mathrm{Ph}-\mathrm{OCH}_{3}\right), 62.8(\mathrm{C}-1)$,

55.1 (1C, 5-O- $\mathrm{CH}_{2}-\mathrm{Ph}-\mathrm{OCH}_{3}$ ), 53.8 (C-5'), 53.8 (C-4'), 37.6 (C-6), 33.5 (C-4),

32.7 (C-2), 28.0 (3C, 2' -NCO-OC $\left.\left(\underline{\mathrm{CH}}_{3}\right)_{3}\right), 27.9$ (3C, 3'-CO-O $\left.\underline{\mathrm{C}}\left(\mathrm{CH}_{3}\right)_{3}\right)$

25.8 (3C, 1-O-Si $\left.\left[\left(\mathrm{CH}_{3}\right)_{2}\right] \mathrm{C}\left(\mathrm{CH}_{3}\right)_{3}\right), 21.2$ (C-3),

$18.2\left(1 \mathrm{C}, 1-\mathrm{O}-\mathrm{Si}\left[\left(\mathrm{CH}_{3}\right)_{2}\right] \underline{\mathrm{C}}\left(\mathrm{CH}_{3}\right)_{3}\right),-5.4\left(2 \mathrm{C}, 1-\mathrm{O}-\mathrm{Si}\left[\left(\mathrm{CH}_{3}\right)_{2}\right] \mathrm{C}\left(\mathrm{CH}_{3}\right)_{3}\right)$

Three signals of C-2', 2'-NCO-OC( $\left(\mathrm{CH}_{3}\right)_{3}$ and 3'- $\underline{\mathrm{CO}}-\mathrm{OC}\left(\mathrm{CH}_{3}\right)_{3}$ were not detected.

HR-MS (FAB, NBA matrix)

$\mathbf{m} / \mathbf{z} 636.4044[\mathrm{M}+\mathrm{H}]^{+}$, Calcd for $\mathrm{C}_{33} \mathrm{H}_{58} \mathrm{~N}_{3} \mathrm{O}_{7} \mathrm{Si} 636.4044[\mathrm{M}+\mathrm{H}]$ 


\section{Scheme 4. Synthesis of (+)-(5R, 4'R)-K01-0509 B (5).}

- $\quad\left(5 R, 4^{\prime} R\right)-6-\left[N, 3^{\prime}\right.$-Bis(tert-butoxycarbonyl)-2' -iminoimidazolidin-4' -yl]-5-( $p$ methoxybenzyloxy)hexan-1-ol (+)-IV

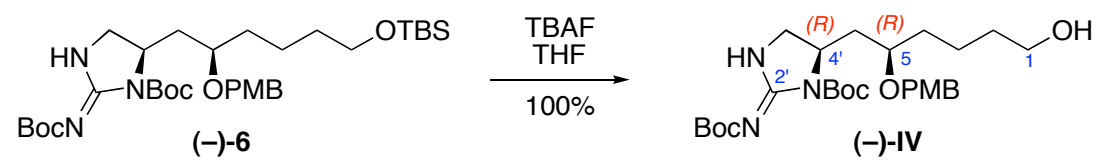

$\left(5 R, 4^{\prime} R\right)$-cyclic guanidine (-)-6 (448 $\left.\mathrm{mg}, 0.705 \mathrm{mmol}\right)$ was dissolved in THF (7.05 $\mathrm{mL})$. To the solution was added TBAF (1.0M THF solution, $2.12 \mathrm{~mL}, 2.12 \mathrm{mmol})$ at room temparature under Ar. After stirring for $2 \mathrm{hr}$, the reaction mixture was added sat. aq. $\mathrm{NH}_{4} \mathrm{Cl}(5.0 \mathrm{~mL})$, and then the organic layer was separated, and the water phase was extracted with $\mathrm{CHCl}_{3}(20 \mathrm{~mL} \mathrm{X} \mathrm{3)}$. The combined organic phase was washed with brine $(10 \mathrm{~mL})$, dried over $\mathrm{Na}_{2} \mathrm{SO}_{4}$, filtered, and evaporated under reduced pressure. Purification by flash chromatography on silica gel $\left(\mathrm{CH}_{3} \mathrm{Cl}: \mathrm{MeOH}=80: 1\right)$ provided the title compound (-)-IV (367 mg, quantitative) as a colorless oil.

$\mathbf{R} \boldsymbol{f}=0.28$ (Silica gel, $\mathrm{CHCl}_{3}: \mathrm{MeOH}=10: 1$ )

$[\alpha]_{\mathbf{D}}{ }^{25}-31.3\left(c 0.84, \mathrm{CHCl}_{3}\right)$

IR $(\mathrm{KBr}) \vee \mathrm{cm}^{-1}$

$1755(\mathrm{C}=\mathrm{O}), 1649(\mathrm{C}=\mathrm{N})$

${ }^{1} \mathrm{H}-\mathrm{NMR}\left(270 \mathrm{MHz}, \mathrm{CDCl}_{3}\right)$

$\delta: 7.22\left(\mathrm{~d}, J=8.6 \mathrm{~Hz}, 2 \mathrm{H}, 5-\mathrm{O}-\mathrm{CH}_{2}-\underline{\mathrm{Ph}}-\mathrm{OCH}_{3}\right)$,

$6.86\left(\mathrm{~d}, \mathrm{~J}=8.6 \mathrm{~Hz}, 2 \mathrm{H}, 5-\mathrm{O}-\mathrm{CH}_{2}-\mathrm{Ph}-\mathrm{OCH}_{3}\right)$,

$4.50\left(\mathrm{~d}, J=11.2 \mathrm{~Hz}, 1 \mathrm{H}, 5-\mathrm{O}-\mathrm{CH}_{2}-\mathrm{Ph}-\mathrm{OCH}_{3}\right)$,

4.30 (d, $\left.J=11.2 \mathrm{~Hz}, 1 \mathrm{H}, 5-\mathrm{O}-\underline{\mathrm{C}}_{2}-\mathrm{Ph}-\mathrm{OCH}_{3}\right), 4.34-4.19$ (m, 1H, 4'-H),

3.80 (s, 3H, 5-O-CH$\left.-\mathrm{Ph}-\mathrm{OC}_{2}{ }_{3}\right), 3.67-3.62\left(\mathrm{~m}, 1 \mathrm{H}, 5^{\prime}-\underline{\mathrm{H}}_{2}\right)$,

3.65 (t, $\left.J=6.3 \mathrm{~Hz}, 2 \mathrm{H}, 1-\mathrm{H}_{2}\right), 3.46-3.37(\mathrm{~m}, 1 \mathrm{H}, 5-\mathrm{H}), 3.43-3.37\left(\mathrm{~m}, 1 \mathrm{H}, 5^{\prime}-\underline{\mathrm{H}}_{2}\right)$,

1.92 (ddd, $\left.J=2.6,8.9,10.9 \mathrm{~Hz}, 1 \mathrm{H}, 6-\underline{\mathrm{H}}_{2}\right), 1.73-1.65\left(\mathrm{~m}, 1 \mathrm{H}, 6-\underline{\mathrm{H}}_{2}\right)$,

1.65-1.55 (m, 2H, 4- $\mathrm{H}_{2}$ ), 1.53-1.36 (complex m, 2H, 2- $\mathrm{H}_{2}$ ),

1.53-1.36 (complex m, 2H, 3- $\left.\mathrm{H}_{2}\right), 1.50$ (s, 9H, 2'-NCO-OC $\left.\left(\mathrm{C}_{3}\right)_{3}\right)$,

1.48 (s, 9H, 3'-CO-OC( $\left.\left(\underline{\mathrm{H}}_{3}\right)_{3}\right)$,

${ }^{13} \mathrm{C}-\mathrm{NMR}\left(67.5 \mathrm{MHz} \mathrm{CDCl}_{3}\right)$

$\delta$ : 159.0 (1C, 5-O- $\left.\mathrm{CH}_{2}-\underline{\mathrm{Ph}}-\mathrm{OCH}_{3}\right), 130.3$ (1C, 5-O- $\mathrm{CH}_{2}-\underline{\mathrm{Ph}}-\mathrm{OCH}_{3}$ ), $129.2\left(2 \mathrm{C}, 5-\mathrm{O}-\mathrm{CH}_{2}-\mathrm{Ph}-\mathrm{OCH}_{3}\right), 113.6$ (2C, 5-O- $\left.\mathrm{CH}_{2}-\mathrm{Ph}-\mathrm{OCH}_{3}\right)$,

82.9 (1C, 2'-NCO-O $\left.\underline{\mathrm{C}}\left(\mathrm{CH}_{3}\right)_{3}\right), 80.4$ (1C, 3'-CO-O $\left.\underline{\mathrm{C}}\left(\mathrm{CH}_{3}\right)_{3}\right)$ 
74.4 (C-5), 70.2 (1C, 5-O- $\left.\mathrm{CH}_{2}-\mathrm{Ph}-\mathrm{OCH}_{3}\right), 62.3$ (C-1), 53.8 (C-5'),

55.1 (1C, 5-O- $\mathrm{CH}_{2}-\mathrm{Ph}-\mathrm{OCH}_{3}$ ), 53.8 (C-4'), 37.6 (C-6), 33.5 (C-4), 32.5 (C-2),

28.0 (3C, 2'-NCO-OC( $\left.\left(\mathrm{CH}_{3}\right)_{3}\right), 27.9$ (3C, 3'-CO-O $\left.\underline{\mathrm{C}}\left(\mathrm{CH}_{3}\right)_{3}\right), 21.2(\mathrm{C}-3)$,

Three signals of C-2', 2'-NCO-OC( $\left(\mathrm{CH}_{3}\right)_{3}$ and 3'- $\underline{\mathrm{CO}}-\mathrm{OC}\left(\mathrm{CH}_{3}\right)_{3}$ were not detected.

HR-MS (FAB, NBA matrix)

$\mathbf{m} / \mathbf{z} 522.3203[\mathrm{M}+\mathrm{H}]^{+}$, Calcd for $\mathrm{C}_{27} \mathrm{H}_{44} \mathrm{~N}_{3} \mathrm{O}_{7} 522.3179[\mathrm{M}+\mathrm{H}]$ 
- $\quad\left(5 R, 4^{\prime} R\right)-6-\left[N, 3^{\prime}\right.$-Bis(tert-butoxycarbonyl)-2'-iminoimidazolidin-4'-yl]-5-( $p$ methoxybenzyloxy)hexanal (-)-18

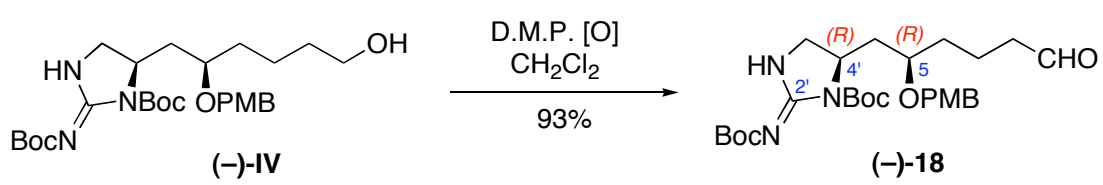

To a solution of $\left(5 R, 4^{\prime} R\right)$-primary alcohol (-)-IV (45.9 $\left.\mathrm{mg}, 88.0 \mu \mathrm{mol}\right)$ in $\mathrm{CH}_{2} \mathrm{Cl}_{2}$ $(1.76 \mathrm{~mL})$ was added Dess-Martin periodinane $(112 \mathrm{mg}, 0.264 \mathrm{mmol})$ at rooom temparature under Ar. After stirring for $30 \mathrm{~min}$, the reaction mixture was quenched with sat. aq. $\mathrm{NaHCO}_{3}(1.0 \mathrm{~mL})$. Then the organic layer was separated, and the water layer was extracted with $\mathrm{CHCl}_{3}(10 \mathrm{~mL} \mathrm{X} \mathrm{3)}$. The combined organic extracts were dried over $\mathrm{Na}_{2} \mathrm{SO}_{4}$, filtered, and evaporated under reduced pressure. Purification by flash chromatography on silica gel $\left(\mathrm{CH}_{3} \mathrm{Cl}: \mathrm{MeOH}=200: 1\right)$ provided the title compound (-)-18 (42.5 mg, 93\%) as a colorless oil.

$\mathbf{R} \boldsymbol{f}=0.48$ (Silica gel, $\left.\mathrm{CHCl}_{3}: \mathrm{MeOH}=10: 1\right)$

$[\alpha]_{\mathbf{D}}{ }^{28}-35.6\left(c 0.44, \mathrm{CHCl}_{3}\right)$

IR $(\mathrm{KBr}) \vee \mathrm{cm}^{-1}$

$1751,1724(\mathrm{C}=\mathrm{O}), 1649(\mathrm{C}=\mathrm{N})$

\section{${ }^{1} \mathrm{H}-\mathrm{NMR}\left(270 \mathrm{MHz} \mathrm{CDCl}_{3}\right.$ )}

$\delta$ : $9.76(\mathrm{~s}, 1 \mathrm{H}, 1-\mathrm{H}), 7.22\left(\mathrm{~d}, J=8.6 \mathrm{~Hz}, 2 \mathrm{H}, 5-\mathrm{O}-\mathrm{CH}_{2}-\mathrm{Ph}-\mathrm{OCH}_{3}\right)$,

$6.86\left(\mathrm{~d}, J=8.6 \mathrm{~Hz}, 2 \mathrm{H}, 5-\mathrm{O}-\mathrm{CH}_{2}-\mathrm{Ph}-\mathrm{OCH}_{3}\right)$,

$4.48\left(\mathrm{~d}, J=11.2 \mathrm{~Hz}, 1 \mathrm{H}, 5-\mathrm{O}-\mathrm{CH}_{2}-\mathrm{Ph}-\mathrm{OCH}_{3}\right)$,

4.30 (d, $\left.J=11.2 \mathrm{~Hz}, 1 \mathrm{H}, 5-\mathrm{O}-\mathrm{C}_{2}-\mathrm{Ph}-\mathrm{OCH}_{3}\right), 4.32-4.25\left(\mathrm{~m}, 1 \mathrm{H}, 4^{\prime}-\mathrm{H}\right)$,

$3.80\left(\mathrm{~s}, 3 \mathrm{H}, 5-\mathrm{O}-\mathrm{CH}_{2}-\mathrm{Ph}-\mathrm{OC}_{\underline{3}}\right), 3.57-3.65\left(\mathrm{~m}, 1 \mathrm{H}, 5^{\prime}-\underline{\mathrm{H}}_{2}\right)$,

3.46-3.39 (complex m, 1H, 5-H), 3.46-3.39 (complex m, 1H, 5'- $\underline{\mathrm{H}}_{2}$ ),

$2.45\left(\mathrm{dt}, J=1.7,7.9 \mathrm{~Hz}, 2 \mathrm{H}, 2-\mathrm{H}_{2}\right), 1.95\left(\mathrm{ddd}, J=2.3,8.6,13.9 \mathrm{~Hz}, 1 \mathrm{H}, 6-\underline{\mathrm{H}}_{2}\right)$,

1.73-1.57 (complex m, 2H, 3- $\mathrm{H}_{2}$ ), 1.73-1.57 (complex m, 2H, 4- $\mathrm{H}_{2}$ ),

1.73-1.57 (complex m, 1H, 6- $\left.\underline{\mathrm{H}}_{2}\right), 1.50$ (s, 9H, 2'-NCO-OC( $\left.\left(\underline{\mathrm{H}}_{3}\right)_{3}\right)$,

1.48 (s, 9H, 3'-CO-OC $\left.\left(\mathrm{C}_{3}\right)_{3}\right)$

${ }^{13} \mathrm{C}-\mathrm{NMR}\left(67.5 \mathrm{MHz} \mathrm{CDCl}_{3}\right)$

$\delta$ : 201.9 (C-1), 159.1 (1C, 5-O- $\left.\mathrm{CH}_{2}-\mathrm{Ph}-\mathrm{OCH}_{3}\right), 130.6$ (1C, 5-O- $\mathrm{CH}_{2}-\mathrm{Ph}-\mathrm{OCH}_{3}$ ),

$129.3\left(2 \mathrm{C}, 5-\mathrm{O}-\mathrm{CH}_{2}-\mathrm{Ph}-\mathrm{OCH}_{3}\right), 113.7$ (2C, 5-O- $\left.\mathrm{CH}_{2}-\mathrm{Ph}-\mathrm{OCH}_{3}\right)$,

83.0 (1C, 2'-NCO-OC $\left.\left(\mathrm{CH}_{3}\right)_{3}\right), 80.4$ (1C, 3'-CO-O $\left.\underline{\mathrm{C}}\left(\mathrm{CH}_{3}\right)_{3}\right), 74.0(\mathrm{C}-5)$,

70.3 (1C, 5-O- $\mathrm{CH}_{2}-\mathrm{Ph}-\mathrm{OCH}_{3}$ ), 55.2 (1C, 5-O- $\left.\mathrm{CH}_{2}-\mathrm{Ph}-\mathrm{OCH}_{3}\right), 53.8$ (C-4'), 53.8 (C-5'), 
43.6 (C-2), 37.6 (C-6), 33.0 (C-4), 28.1 (3C, 2'-NCO-OC $\left(\mathrm{CH}_{3}\right)_{3}$ ),

28.0 (3C, 3'-CO-OC $\left.\left(\mathrm{CH}_{3}\right)_{3}\right), 17.4$ (C-3)

Three signals of C-2', 2'-NCO-OC( $\left(\mathrm{CH}_{3}\right)_{3}$ and 3'- $\underline{\mathrm{CO}}-\mathrm{OC}\left(\mathrm{CH}_{3}\right)_{3}$ were not detected.

HR-MS (FAB, NBA matrix)

$\mathbf{m} / \mathbf{z} 520.3015[\mathrm{M}+\mathrm{H}]^{+}$, Calcd for $\mathrm{C}_{27} \mathrm{H}_{42} \mathrm{~N}_{3} \mathrm{O}_{7} 520.3023[\mathrm{M}+\mathrm{H}]$ 
- $\quad\left(5 R, 4^{\prime} R\right)-6-\left[N, 3^{\prime}\right.$-Bis(tert-butoxycarbonyl)-1' -( $p$-methoxyphenylcarbamoyl)-2'iminoimidazolidin-4'-yl]-5-( $p$-methoxybenzyloxy)hexanal (-)-V

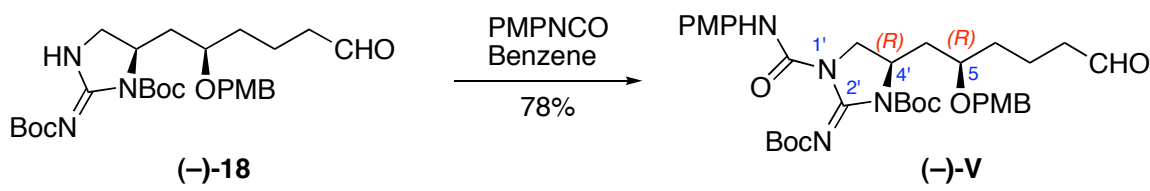

To solution of aldehyde (-)-18 $(42.5 \mathrm{mg}, 0.082 \mathrm{mmol})$ in benzene $(818 \mu \mathrm{L})$ was added $p$-methoxyphenyl isocyanate $(15.9 \mu \mathrm{g}, 0.123 \mathrm{mmol})$ at room temprature. After stirring for $30 \mathrm{~min}$, the reaction mixture was added sat. $\mathrm{NH}_{4} \mathrm{Cl}(1.0 \mathrm{~mL})$, then the two layers were separated and the aqueous layer was extracted with $\mathrm{CHCl}_{3}(10 \mathrm{~mL} \mathrm{X} \mathrm{3})$. The combined organic extracts were dried over $\mathrm{Na}_{2} \mathrm{SO}_{4}$, filtered, and evaporated under reduced pressure. Purification by flash chromatography on silica gel $\left(\mathrm{CH}_{3} \mathrm{Cl}: \mathrm{MeOH}=\right.$ $200: 1)$ provided the title compound (-)-V (42.4 mg, 78\%) as a colorless oil.

$\mathbf{R} \boldsymbol{f}=0.53$ (Silica gel, $\mathrm{CHCl}_{3}: \mathrm{MeOH}=10: 1$ )

$[\alpha]_{\mathrm{D}}{ }^{30}-62.2\left(c 0.70, \mathrm{CHCl}_{3}\right)$

IR $(\mathrm{KBr}) \vee \mathrm{cm}^{-1}$

$1753(\mathrm{C}=\mathrm{O}), 1693(\mathrm{C}=\mathrm{N})$

\section{${ }^{1} \mathrm{H}-\mathrm{NMR}\left(270 \mathrm{MHz}, \mathrm{CDCl}_{3}\right)$}

$\delta: 10.9$ (s, 1H, 1'-CO-N $\left.\underline{H}-\mathrm{Ph}-\mathrm{OCH}_{3}\right), 9.72(\mathrm{~s}, 1 \mathrm{H}, 1-\mathrm{H})$,

$7.33\left(\mathrm{~d}, J=8.6 \mathrm{~Hz}, 2 \mathrm{H}, 1\right.$ ' $\left.-\mathrm{CO}-\mathrm{NH}-\mathrm{Ph}-\mathrm{OCH}_{3}\right)$,

$7.20\left(\mathrm{~d}, J=8.3 \mathrm{~Hz}, 2 \mathrm{H}, 5-\mathrm{O}-\mathrm{CH}_{2}-\mathrm{Ph}-\mathrm{OCH}_{3}\right)$,

$6.83\left(\mathrm{~d}, J=8.6 \mathrm{~Hz}, 2 \mathrm{H}, 1^{\prime}-\mathrm{CO}-\mathrm{NH}-\mathrm{Ph}-\mathrm{OCH}_{3}\right)$,

$6.80\left(\mathrm{~d}, J=8.6 \mathrm{~Hz}, 2 \mathrm{H}, 5-\mathrm{O}-\mathrm{CH}_{2}-\mathrm{Ph}-\mathrm{OCH}_{3}\right)$,

$4.49\left(\mathrm{~d}, J=11.1 \mathrm{~Hz}, 1 \mathrm{H}, 5-\mathrm{O}-\mathrm{CH}_{2}-\mathrm{Ph}-\mathrm{OCH}_{3}\right)$,

4.29 (d, $\left.J=11.1 \mathrm{~Hz}, 1 \mathrm{H}, 5-\mathrm{O}-\mathrm{C}_{2}-\mathrm{Ph}-\mathrm{OCH}_{3}\right), 4.36-4.27$ (m, 1H, 4'-H),

$3.90\left(\mathrm{dd}, J=1.3,10.9 \mathrm{~Hz}, 1 \mathrm{H}, 5^{\prime}-\underline{\mathrm{H}}_{2}\right), 3.76$ (s, 3H, 1'-CO-NH-Ph-OC $\left.\underline{\mathrm{H}}_{3}\right)$,

3.72 (s, 3H, 5-O- $\left.\mathrm{CH}_{2}-\mathrm{Ph}-\mathrm{OC}_{\underline{3}}\right), 3.63$ (dd, $\left.J=8.6,10.9 \mathrm{~Hz}, 1 \mathrm{H}, 5^{\prime}-\underline{\mathrm{H}}_{2}\right)$,

3.56-3.49 (m, 1H, 5-H), 2.43 (dt, $J=1.3,6.3 \mathrm{~Hz}, 2 \mathrm{H}, 2-\mathrm{H}_{2}$ ),

1.99 (ddd, $\left.J=2.3,9.2,13.8 \mathrm{~Hz}, 1 \mathrm{H}, 6-\underline{\mathrm{H}}_{2}\right), 1.78$ (ddd, $\left.J=3.0,9.5,13.8 \mathrm{~Hz}, 1 \mathrm{H}, 6-\underline{\mathrm{H}}_{2}\right)$,

1.69-1.57 (m, 2H, 3- $\left.\mathrm{H}_{2}\right), 1.69-1.57\left(\mathrm{~m}, 2 \mathrm{H}, 4-\underline{\mathrm{H}}_{2}\right)$,

1.52 (s, 9H, 2'-NCO-OC( $\left.\left(\underline{\mathrm{H}}_{3}\right)_{3}\right), 1.48$ (s, 9H, 3'-CO-OC( $\left.\left.\mathrm{C}_{3}\right)_{3}\right)$,

${ }^{13} \mathrm{C}-\mathrm{NMR}\left(67.5 \mathrm{MHz} \mathrm{CDCl}_{3}\right)$

$\delta$ : 201.9 (C-1), 159.2 (1C, 5-O- $\mathrm{CH}_{2}-\mathrm{Ph}-\mathrm{OCH}_{3}$ ), 157.1 (C-2'),

156.3 (1C, 1'-CO-NH- $\left.\underline{\mathrm{Ph}}-\mathrm{OCH}_{3}\right), 150.2$ (1C, 2'-NCO-OC( $\left.\left.\mathrm{CH}_{3}\right)_{3}\right)$, 


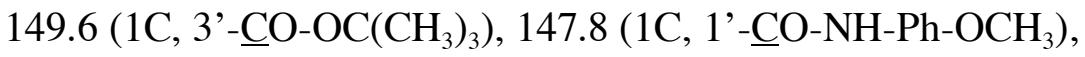

130.6 (1C, 1'-CO-NH- $\left.-\mathrm{Ph}-\mathrm{OCH}_{3}\right)$

130.2 (1C, 5-O- $\left.\mathrm{CH}_{2}-\mathrm{Ph}-\mathrm{OCH}_{3}\right), 129.3$ (2C, 5-O- $\mathrm{CH}_{2}-\mathrm{Ph}-\mathrm{OCH}_{3}$ ),

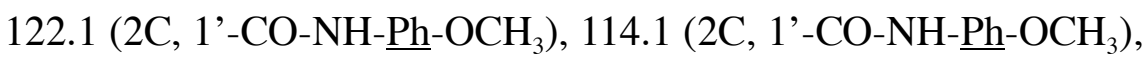

113.8 (2C, 5-O-CH - - $\left.\mathrm{Ph}-\mathrm{OCH}_{3}\right), 83.9$ (1C, 2'-NCO-O $\left.\underline{(}\left(\mathrm{CH}_{3}\right)_{3}\right)$,

80.5 (1C, 3'-CO-OC $\left.\left(\mathrm{CH}_{3}\right)_{3}\right), 73.9$ (C-5), $70.4\left(1 \mathrm{C}, 5-\mathrm{O}-\mathrm{CH}_{2}-\mathrm{Ph}-\mathrm{OCH}_{3}\right)$,

55.4 (1C, 1'-CO-NH-Ph-O $\underline{C H}_{3}$ ), 55.2 (1C, 5-O- $\mathrm{CH}_{2}-\mathrm{Ph}-\mathrm{OCH}_{3}$ ), 52.3 (C-4'),

45.9 (C-5'), 43.7 (C-2), 38.0 (C-6), 32.8 (C-4),

28.1 (3C, 2'-NCO-OC $\left.\left(\mathrm{CH}_{3}\right)_{3}\right), 28.0$ (3C, 3'-CO-OC $\left.\left(\mathrm{CH}_{3}\right)_{3}\right), 17.2$ (C-3),

HR-MS (FAB, NBA matrix)

$\mathbf{m} / \mathbf{z} 669.3471[\mathrm{M}+\mathrm{H}]^{+}$, Calcd for $\mathrm{C}_{35} \mathrm{H}_{49} \mathrm{~N}_{4} \mathrm{O}_{9} 669.3500[\mathrm{M}+\mathrm{H}]$ 
- $\quad\left(5 R, 4^{\prime} R\right)-6-[N, 3$ '-Bis (tert-butoxycarbonyl)-1' -( $p$-methoxyphenylcarbamoyl)-2'iminoimidazolidin-4'-yl]-5-( $p$-methoxybenzyloxy)hexanoic acid (-)-19
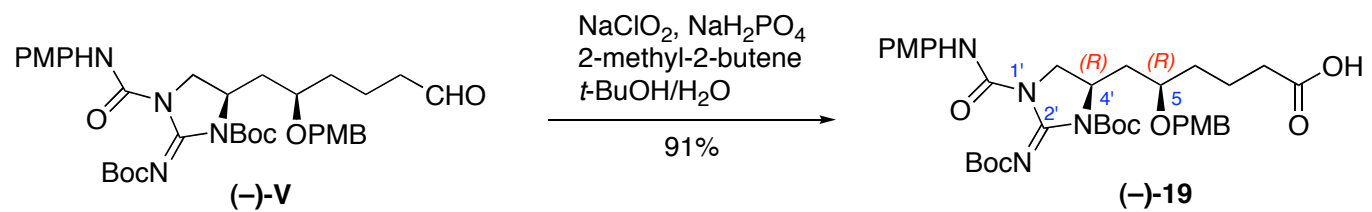

Aldehyde (-)-V (210 mg, $0.314 \mathrm{mmol})$ was dissolved in $t$ - $\mathrm{BuOH}(818 \mathrm{~mL})$ and then added 2-methyl-2-butene $(665 \mu \mathrm{L}, 6.28 \mathrm{mmol})$. And then the solution of $\mathrm{NaClO}_{2}(284$ $\mathrm{mg}, 3.14 \mathrm{mmol})$ and $\mathrm{NaH}_{2} \mathrm{PO}_{4}(634 \mathrm{mg}, 4.08 \mathrm{mmol})$ in $\mathrm{H}_{2} \mathrm{O}(6.3 \mathrm{~mL})$ was added to the mixture. After stirring for $10 \mathrm{~min}$, the reaction solution was diluted with $\mathrm{CHCl}_{3}(10 \mathrm{~mL})$ and sat. aq. $\mathrm{NH}_{4} \mathrm{Cl}(5 \mathrm{~mL})$ was added to the reaction mixture. Then the solution was extracted with $\mathrm{CHCl}_{3}(15 \mathrm{~mL} \mathrm{X} \mathrm{3)}$. The combined organic extracts were dried over $\mathrm{Na}_{2} \mathrm{SO}_{4}$, filtered, and evaporated under reduced pressure. Flash chromatography $\left(\mathrm{CHCl}_{3}: \mathrm{MeOH}=30: 1\right)$ was carried out to get the pure title compound (-)-19 (195 mg, $91 \%$ ) as a pale orange powder.

$\mathbf{R} \boldsymbol{f}=0.35$ (Silica gel, $\mathrm{CHCl}_{3}: \mathrm{MeOH}=10: 1$ )

$[\alpha]_{\mathrm{D}}{ }^{27}-63.8\left(c 0.45, \mathrm{CHCl}_{3}\right)$

IR $(\mathrm{KBr}) \vee \mathrm{cm}^{-1}$

$1752(\mathrm{C}=\mathrm{O}), 1698(\mathrm{C}=\mathrm{N})$

\section{${ }^{1} \mathrm{H}-\mathrm{NMR}\left(300 \mathrm{MHz}, \mathrm{CDCl}_{3}\right.$ )}

$\delta$ : 10.9 (s, 1H, 1'-CO-NH-Ph-OCH ${ }_{3}$ ), 7.34 (d, $J=8.4 \mathrm{~Hz}, 2 \mathrm{H}, 1$ '-CO-NH-Ph- $\mathrm{OCH}_{3}$ ),

$7.21\left(\mathrm{~d}, J=8.9 \mathrm{~Hz}, 2 \mathrm{H}, 5-\mathrm{O}-\mathrm{CH}_{2}-\mathrm{Ph}-\mathrm{OCH}_{3}\right)$,

$6.84\left(\mathrm{~d}, J=8.4 \mathrm{~Hz}, 2 \mathrm{H}, 1^{\prime}-\mathrm{CO}-\mathrm{NH}-\mathrm{Ph}-\mathrm{OCH}_{3}\right)$,

$6.82\left(\mathrm{~d}, J=8.9 \mathrm{~Hz}, 2 \mathrm{H}, 5-\mathrm{O}-\mathrm{CH}_{2}-\mathrm{Ph}-\mathrm{OCH}_{3}\right)$,

$4.51\left(\mathrm{~d}, J=11.0 \mathrm{~Hz}, 1 \mathrm{H}, 5-\mathrm{O}-\mathrm{CH}_{2}-\mathrm{Ph}-\mathrm{OCH}_{3}\right)$,

4.29 (d, $\left.J=11.0 \mathrm{~Hz}, 1 \mathrm{H}, 5-\mathrm{O}-\underline{\mathrm{C}}_{2}-\mathrm{Ph}-\mathrm{OCH}_{3}\right), 4.34-4.29$ (m, $\left.1 \mathrm{H}, 4^{\prime}-\mathrm{H}\right)$,

$3.91\left(\mathrm{dd}, J=2.0,11.0 \mathrm{~Hz}, 1 \mathrm{H}, 5^{\prime}-\underline{\mathrm{H}}_{2}\right), 3.77$ (s, 3H, 1'-CO-NH-Ph-OC $\left.\underline{\mathrm{H}}_{3}\right)$,

3.73 (s, 3H, 5-O-CH$\left.-\mathrm{Ph}-\mathrm{OC}_{2} \underline{H}_{2}\right), 3.63$ (dd, $\left.J=8.5,11.0 \mathrm{~Hz}, 1 \mathrm{H}, 5^{\prime}-\underline{\mathrm{H}}_{2}\right)$,

$3.58-3.51(\mathrm{~m}, 1 \mathrm{H}, 5-\mathrm{H}), 2.35\left(\mathrm{t}, J=6.5 \mathrm{~Hz}, 2 \mathrm{H}, 2-\mathrm{H}_{2}\right)$,

1.99 (ddd, $\left.J=2.5,9.8,14.0 \mathrm{~Hz}, 1 \mathrm{H}, 6-\underline{\mathrm{H}}_{2}\right), 1.78\left(\mathrm{ddd}, J=3.0,9.5,14.0 \mathrm{~Hz}, 1 \mathrm{H}, 6-\underline{\mathrm{H}}_{2}\right)$,

$1.69-1.58\left(\mathrm{~m}, 2 \mathrm{H}, 3-\mathrm{H}_{2}\right), 1.69-1.58\left(\mathrm{~m}, 2 \mathrm{H}, 4-\mathrm{H}_{2}\right)$,

1.52 (s, 9H, 2'-NCO-OC( $\left.\left(\underline{\mathrm{H}}_{3}\right)_{3}\right), 1.48$ (s, 9H, 3'-CO-OC $\left.\left(\mathrm{CH}_{3}\right)_{3}\right)$,

${ }^{13} \mathrm{C}-\mathrm{NMR}$ (75.0 $\mathrm{MHz}, \mathrm{CDCl}_{3}$ ) 
$\delta: 178.1$ (C-1), 159.1 (1C, 5-O- $\left.\mathrm{CH}_{2}-\mathrm{Ph}-\mathrm{OCH}_{3}\right), 157.1$ (C-2'),

156.2 (1C, 1'-CO-NH-Ph-OCH 3 ), $150.3\left(1 \mathrm{C}, 2^{\prime}\right.$ - $\left.\mathrm{N} \underline{\mathrm{CO}}-\mathrm{OC}\left(\mathrm{CH}_{3}\right)_{3}\right)$,

$149.5\left(1 \mathrm{C}, 3^{\prime}-\mathrm{CO}-\mathrm{OC}\left(\mathrm{CH}_{3}\right)_{3}\right), 147.6\left(1 \mathrm{C}, 1^{\prime}-\mathrm{CO}-\mathrm{NH}-\mathrm{Ph}-\mathrm{OCH}_{3}\right)$,

130.5 (1C, 1'-CO-NH-Ph- $\left.-\mathrm{OCH}_{3}\right), 130.2$ (1C, 5-O- $\left.\mathrm{CH}_{2}-\mathrm{Ph}-\mathrm{OCH}_{3}\right)$,

129.2 (2C, 5-O- $\left.\mathrm{CH}_{2}-\mathrm{Ph}-\mathrm{OCH}_{3}\right), 122.1$ (2C, 1'-CO-NH- $\underline{\mathrm{Ph}}-\mathrm{OCH}_{3}$ ),

114.0 (2C, 1'-CO-NH- $\left.\mathrm{Ph}-\mathrm{OCH}_{3}\right), 113.7$ (2C, 5-O- $\left.\mathrm{CH}_{2}-\mathrm{Ph}-\mathrm{OCH}_{3}\right)$,

83.9 (1C, 2'-NCO-O $\left.\underline{\mathrm{C}}\left(\mathrm{CH}_{3}\right)_{3}\right), 80.5\left(1 \mathrm{C}, 3^{\prime}\right.$ - $\left.\mathrm{CO}-\mathrm{O} \underline{\mathrm{C}}\left(\mathrm{CH}_{3}\right)_{3}\right)$,

73.7 (C-5), 70.3 (1C, 5-O- $\mathrm{CH}_{2}-\mathrm{Ph}-\mathrm{OCH}_{3}$ ), 55.3 (1C, 1'-CO-NH-Ph-OCH${ }_{3}$ ),

55.1 (1C, 5-O- $\mathrm{CH}_{2}-\mathrm{Ph}-\mathrm{OCH}_{3}$ ), 52.2 (C-4'), 45.8 (C-5'), 37.9 (C-6), 33.8 (C-2),

32.7 (C-4), 28.0 (3C, 2'-NCO-OC( $\left.\left(\mathrm{CH}_{3}\right)_{3}\right), 27.9$ (3C, 3'-CO-OC( $\left.\left(\mathrm{CH}_{3}\right)_{3}\right), 19.8(\mathrm{C}-3)$

HR-MS (FAB, NBA matrix)

$\mathbf{m} / \mathbf{z} 685.3468[\mathrm{M}+\mathrm{H}]^{+}$, Calcd for $\mathrm{C}_{35} \mathrm{H}_{49} \mathrm{~N}_{4} \mathrm{O}_{9} 685.3449[\mathrm{M}+\mathrm{H}]$ 
- $\quad\left(5 R, 4^{\prime} R\right)-6-[N, 3$ '-Bis (tert-butoxycarbonyl)-1' -carbamoyl-2'-iminoimidazolidin4'-yl]-5-(p-methoxybenzyloxy)hexanoic acid (-)-VI

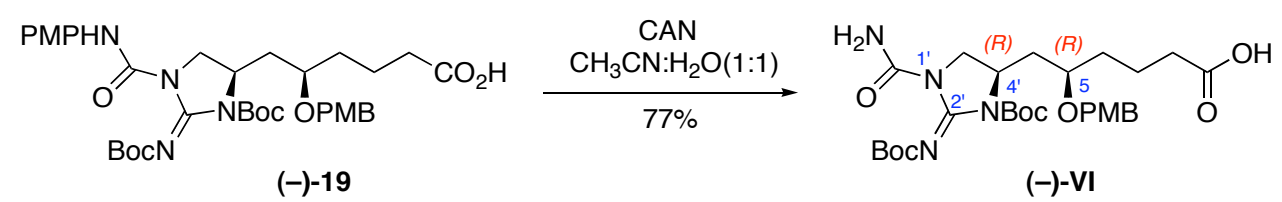

To solution of carboxylic acid (-)-19 (70.0 mg, $0.102 \mathrm{mmol})$ in $\mathrm{CH}_{3} \mathrm{CN}: \mathrm{H}_{2} \mathrm{O}(1: 1)$ $(4.08 \mathrm{~mL})$ was added cerium (IV) ammonium nitrate $(140.3 \mathrm{mg}, 0.256 \mathrm{mmol})$ at $0^{\circ} \mathrm{C}$. After stirring for $10 \mathrm{~min}$, the reaction mixture was added $\mathrm{CHCl}_{3}(10 \mathrm{~mL})$. The two

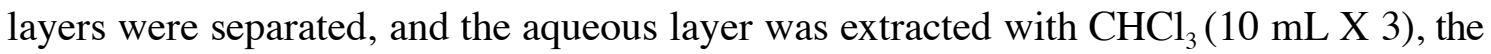
combined organic extracts were washed with $\mathrm{H}_{2} \mathrm{O}(5.0 \mathrm{~mL} \mathrm{X} \mathrm{3})$, brine $(5.0 \mathrm{~mL})$ and dried over $\mathrm{Na}_{2} \mathrm{SO}_{4}$, filtered, and evaporated under reduced pressure. Purification by flash chromatography on silica gel $\left(\mathrm{CH}_{3} \mathrm{Cl}: \mathrm{MeOH}=30: 1\right)$ provided the title compound VI (45.6 mg, 77\%) as a colorless oil.

$\mathbf{R} \boldsymbol{f}=0.25$ (Silica gel, $\mathrm{CHCl}_{3}: \mathrm{MeOH}=10: 1$ )

$[\alpha]_{\mathrm{D}}{ }^{26}-42.6\left(c 0.44, \mathrm{CHCl}_{3}\right)$

IR $(\mathrm{KBr}) \vee \mathrm{cm}^{-1}$

$1755(\mathrm{C}=\mathrm{O}), 1709(\mathrm{C}=\mathrm{N})$

\section{${ }^{1} \mathrm{H}-\mathrm{NMR}\left(270 \mathrm{MHz}, \mathrm{CDCl}_{3}\right)$}

$\delta: 8.75,5.71$ (s, each 1H, 1'-CO-NH$\left.{ }_{2}\right), 7.22\left(\mathrm{~d}, J=8.6 \mathrm{~Hz}, 2 \mathrm{H}, 5-\mathrm{O}-\mathrm{CH}_{2}-\underline{\mathrm{Ph}}-\mathrm{OCH}_{3}\right)$,

$6.86\left(\mathrm{~d}, \mathrm{~J}=8.6 \mathrm{~Hz}, 2 \mathrm{H}, 5-\mathrm{O}-\mathrm{CH}_{2}-\mathrm{Ph}-\mathrm{OCH}_{3}\right)$,

$4.51\left(\mathrm{~d}, J=11.2 \mathrm{~Hz}, 1 \mathrm{H}, 5-\mathrm{O}-\mathrm{CH}_{2}-\mathrm{Ph}-\mathrm{OCH}_{3}\right)$,

$4.28\left(\mathrm{~d}, J=11.0 \mathrm{~Hz}, 1 \mathrm{H}, 5-\mathrm{O}-\mathrm{C}_{2}-\mathrm{Ph}-\mathrm{OCH}_{3}\right), 4.27-4.23\left(\mathrm{~m}, 1 \mathrm{H}, 4^{\prime}-\mathrm{H}\right)$,

3.85-3.75 (m, 1H, 5'- $\left.\underline{\mathrm{H}}_{2}\right), 3.79$ (s, 3H, 5-O- $\left.\mathrm{CH}_{2}-\mathrm{Ph}-\mathrm{OC}_{\underline{3}}\right)$,

$3.54\left(\mathrm{dd}, J=8.2,10.9 \mathrm{~Hz}, 1 \mathrm{H}, 5^{\prime}-\underline{\mathrm{H}}_{2}\right)$,

3.54-3.45 (m, 1H, 5-H), $2.36\left(\mathrm{t}, J=6.3 \mathrm{~Hz}, 2 \mathrm{H}, 2-\mathrm{H}_{2}\right)$,

1.97 (ddd, $J=2.6,9.2,14.2 \mathrm{~Hz}, 1 \mathrm{H}, 6-\underline{\mathrm{H}}_{2}$ ), 1.74 (ddd, $J=2.5,9.8,14.2 \mathrm{~Hz}, 1 \mathrm{H}, 6-\underline{\mathrm{H}}_{2}$ ),

1.70-1.57 (m, 2H, 3- $\left.\mathrm{H}_{2}\right), 1.70-1.57\left(\mathrm{~m}, 2 \mathrm{H}, 4-\mathrm{H}_{2}\right)$,

$1.50\left(\mathrm{~s}, 9 \mathrm{H}, 2^{\prime}-\mathrm{NCO}-\mathrm{OC}\left(\mathrm{CH}_{3}\right)_{3}\right), 1.48$ (s, 9H, 3'-CO-OC $\left.\left(\mathrm{C}_{3}\right)_{3}\right)$

\section{${ }^{13} \mathrm{C}-\mathrm{NMR}\left(67.5 \mathrm{MHz}, \mathrm{CDCl}_{3}\right)$}

$\delta$ : 177.6 (C-1), 159.2 (1C, 5-O- $\left.\mathrm{CH}_{2}-\mathrm{Ph}-\mathrm{OCH}_{3}\right), 157.3$ (C-2'),

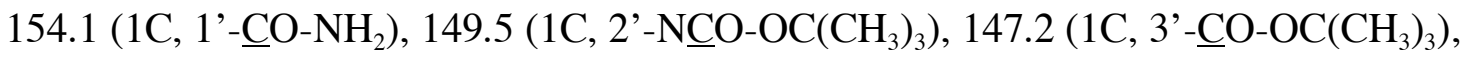
$130.3\left(1 \mathrm{C}, 5-\mathrm{O}-\mathrm{CH}_{2}-\mathrm{Ph}-\mathrm{OCH}_{3}\right), 129.4\left(2 \mathrm{C}, 5-\mathrm{O}-\mathrm{CH}_{2}-\underline{\mathrm{Ph}}-\mathrm{OCH}_{3}\right)$, 
$113.8\left(2 \mathrm{C}, 5-\mathrm{O}-\mathrm{CH}_{2}-\mathrm{Ph}-\mathrm{OCH}_{3}\right), 83.9\left(1 \mathrm{C}, 2^{\prime}-\mathrm{NCO}-\mathrm{O} \underline{\mathrm{C}}\left(\mathrm{CH}_{3}\right)_{3}\right)$,

80.5 (1C, 3'-CO-OC( $\left.\left(\mathrm{CH}_{3}\right)_{3}\right), 73.9(\mathrm{C}-5), 70.5$ (1C, 5-O- $\left.\mathrm{CH}_{2}-\mathrm{Ph}-\mathrm{OCH}_{3}\right)$,

55.2 (1C, 5-O- $\mathrm{CH}_{2}-\mathrm{Ph}-\mathrm{OCH}_{3}$ ), 52.4 (C-4'), 45.5 (C-5'), 37.9 (C-6), 33.9 (C-2),

32.9 (C-4), 28.1 (3C, 2'-NCO-OC $\left.\left(\mathrm{CH}_{3}\right)_{3}\right), 28.0$ (3C, 3'-CO-O $\left.\underline{\mathrm{C}}\left(\mathrm{CH}_{3}\right)_{3}\right), 20.0(\mathrm{C}-3)$

HR-MS (FAB, NBA matrix)

$\mathbf{m} / \mathbf{z} 579.3047[\mathrm{M}+\mathrm{H}]^{+}$, Calcd for $\mathrm{C}_{28} \mathrm{H}_{43} \mathrm{~N}_{4} \mathrm{O}_{9} 579.3030[\mathrm{M}+\mathrm{H}]$ 


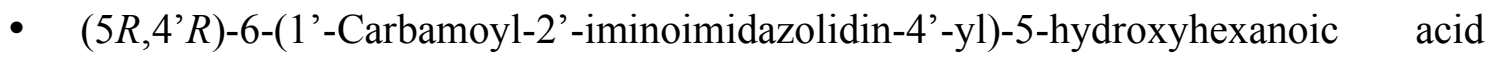
(+)-(5R, 4'R)-K01-0509 B (5)

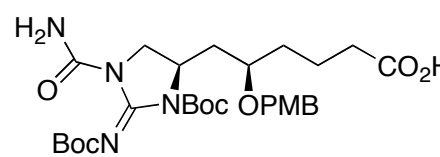

$(-)-\mathrm{VI}$

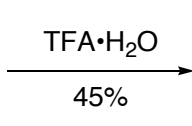

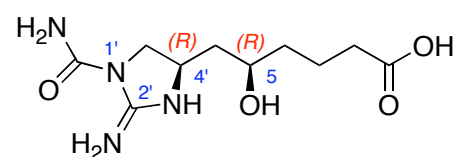

(+)-(5R, 4'R)-K01-0509 B (5)

The carboxylic acid (-)-VI (17.5 mg, $0.030 \mathrm{mmol})$ was dissolved in TFA : $\mathrm{H}_{2} \mathrm{O}(3: 1)$ $(1.50 \mathrm{~mL})$ and the reaction mixture was stirred for $1 \mathrm{hr}$ at room temparature. The reaction mixture was diluted by $\mathrm{H}_{2} \mathrm{O}(5.0 \mathrm{~mL})$, and then the mixture was evaporated to remove the solvent. The crude product was purified by preparative HPLC (Senshu Pak PEGASIL ODS $20 \phi \times 250 \mathrm{~mm}, \mathrm{MeOH}: \mathrm{H}_{2} \mathrm{O}(2: 98), 7.0 \mathrm{~mL} / \mathrm{min}$, UV at $\left.210 \mathrm{~nm}\right)$ and provided the desired compound (+)-(5R, 4'R)-K01-0509 B (5) $(3.5 \mathrm{mg}, 45 \%)$.

$[\alpha]_{\mathrm{D}}^{25}+32.4(c 0.16, \mathrm{MeOH})$

IR $(\mathrm{KBr}) \vee \mathrm{cm}^{-1}$

$1731(\mathrm{C}=\mathrm{O}), 1681(\mathrm{C}=\mathrm{N})$

\section{${ }^{1} \mathrm{H}-\mathrm{NMR}\left(400 \mathrm{MHz}, \mathrm{D}_{2} \mathrm{O}\right)$}

$\delta$ : 4.26-4.21 (m, 1H, 4'-H), 4.19 (dd, $\left.J=10.0,15.0 \mathrm{~Hz}, 1 \mathrm{H}, 5^{\prime}-\underline{\mathrm{H}}_{2}\right)$

$3.78\left(\mathrm{dd}, J=1.0,15.0 \mathrm{~Hz}, 1 \mathrm{H}, 5\right.$ ' $\left.-\underline{\mathrm{H}}_{2}\right), 3.77-3.70(\mathrm{~m}, 1 \mathrm{H}, 5-\mathrm{H})$,

2.26 (t, $\left.J=7.2 \mathrm{~Hz}, 2 \mathrm{H}, 2-\mathrm{H}_{2}\right), 1.83$ (ddd, $J=4.0,6.0,14.0 \mathrm{~Hz}, 1 \mathrm{H}, 6-\underline{\mathrm{H}}_{2}$ ),

1.76 (ddd, $\left.J=6.0,9.0,14.0 \mathrm{~Hz}, 1 \mathrm{H}, 6-\underline{\mathrm{H}}_{2}\right), 1.69-1.59$ (m, $\left.1 \mathrm{H}, 3-\underline{\mathrm{H}}_{2}\right)$,

1.59-1.50 (m, 1H, 3- $\underline{\mathrm{H}}_{2}$ ), 1.49-1.43 (m, 2H, 4- $\mathrm{H}_{2}$ )

${ }^{13} \mathrm{C}-\mathrm{NMR}\left(75 \mathrm{MHz}, \mathrm{D}_{2} \mathrm{O}\right)$

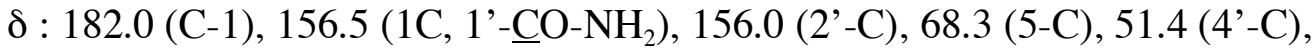

50.6 (5'-C), 41.3 (6-C), 36.4 (4-C), 36.0 (2-C), 21.3 (3-C)

HR-MS (FAB, NBA matrix)

$\mathbf{m} / \mathbf{z} 259.1404[\mathrm{M}+\mathrm{H}]^{+}$, Calcd for $\mathrm{C}_{10} \mathrm{H}_{19} \mathrm{~N}_{4} \mathrm{O}_{4} 259.1406[\mathrm{M}+\mathrm{H}]$ 


\section{Scheme 5. Synthesis of $(5 R, 4 ' S)$-K01-0509 B (10).}

$(2 R, 4 R)-1-\left[N{ }^{\prime}, N\right.$ ' -Bis(tert-butoxycarbonyl)guanidino]-8-(tert-butyldimethylsiloxy)-4-( p-methoxybenzyloxy)octan-2-ol (-)-20

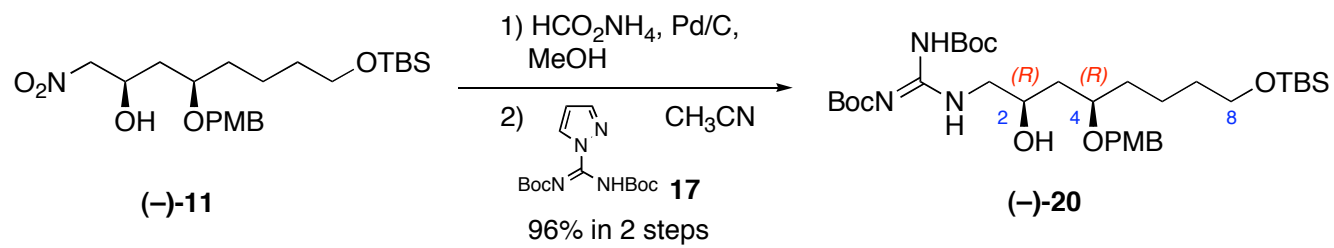

According to the procedure of preparation of the compound (-)-7, the title compound (-)-20 (331 mg, 96\% in 2 steps) was obtained from the alcohol (-)-11 (232 mg, 0.525 mmol).

$\mathbf{R f}=0.50($ Silica gel, Hexane $:$ EtOAc $=2: 1)$

$[\alpha]_{\mathbf{D}}{ }^{23}-29.7\left(c 0.82, \mathrm{CHCl}_{3}\right)$

IR $(\mathrm{KBr}) \vee \mathrm{cm}^{-1}$

$3332(\mathrm{~N}-\mathrm{H}), 1724(\mathrm{C}=\mathrm{O}), 1641(\mathrm{C}=\mathrm{N})$

\section{${ }^{1} \mathrm{H}-\mathrm{NMR}\left(400 \mathrm{MHz}, \mathrm{CDCl}_{3}\right.$ )}

$\delta: 11.47\left(\mathrm{~s}, 1 \mathrm{H}, 1-\mathrm{NH}-\mathrm{C}\left[\mathrm{NHCO}-\mathrm{OC}\left(\mathrm{CH}_{3}\right)_{3}\right]\left[\mathrm{NCO}-\mathrm{OC}\left(\mathrm{CH}_{3}\right)_{3}\right]\right)$,

$8.67\left(\mathrm{dd}, 1 \mathrm{H}, J=4.9,6.0 \mathrm{~Hz}, 1-\mathrm{N} \underline{\mathrm{H}}-\mathrm{C}\left[\mathrm{NHCO}-\mathrm{OC}\left(\mathrm{CH}_{3}\right)_{3}\right]\left[\mathrm{NCO}-\mathrm{OC}\left(\mathrm{CH}_{3}\right)_{3}\right]\right)$,

$7.25\left(\mathrm{~d}, J=8.6 \mathrm{~Hz}, 2 \mathrm{H}, 4-\mathrm{O}-\mathrm{CH}_{2}-\mathrm{Ph}-\mathrm{OCH}_{3}\right)$,

$6.87\left(\mathrm{~d}, J=8.6 \mathrm{~Hz}, 2 \mathrm{H}, 4-\mathrm{O}-\mathrm{CH}_{2}-\mathrm{Ph}-\mathrm{OCH}_{3}\right)$,

$4.55\left(\mathrm{~d}, J=11.0 \mathrm{~Hz}, 1 \mathrm{H}, 4-\mathrm{O}-\mathrm{C}_{2}-\mathrm{Ph}-\mathrm{OCH}_{3}\right)$,

$4.36\left(\mathrm{~d}, J=11.0 \mathrm{~Hz}, 1 \mathrm{H}, 4-\mathrm{O}-\mathrm{C}_{2}-\mathrm{Ph}-\mathrm{OCH}_{3}\right), 3.95-3.89$ (m, $\left.1 \mathrm{H}, 2-\mathrm{H}\right)$,

$3.80\left(\mathrm{~s}, 3 \mathrm{H}, 4-\mathrm{O}-\mathrm{CH}_{2}-\mathrm{Ph}-\mathrm{OCH}_{3}\right), 3.70-3.62(\mathrm{~m}, 1 \mathrm{H}, 4-\mathrm{H}), 3.61\left(\mathrm{t}, J=6.1 \mathrm{~Hz}, 2 \mathrm{H}, 8-\mathrm{H}_{2}\right)$,

$3.60\left(\mathrm{ddd}, J=3.0,6.0,13.5 \mathrm{~Hz}, 1 \mathrm{H}, 1-\underline{\mathrm{H}}_{2}\right), 3.30\left(\mathrm{ddd}, J=4.9,7.5,13.5 \mathrm{~Hz}, 1 \mathrm{H}, 1-\underline{\mathrm{H}}_{2}\right)$,

1.71 (ddd, $\left.J=9.0,9.0,14.5 \mathrm{~Hz}, 1 \mathrm{H}, 3-\underline{\mathrm{H}}_{2}\right)$,

1.63 (dddd, $J=2.0,3.0,7.0,14.0 \mathrm{~Hz}, 1 \mathrm{H}, 5-\underline{\mathrm{H}}_{2}$ ),

1.60 (dddd, $J=3.0,7.0,10.0,14.0 \mathrm{~Hz}, 1 \mathrm{H}, 5-\underline{\mathrm{H}}_{2}$ ),

1.53 (ddd, $\left.J=3.5,4.0,14.5 \mathrm{~Hz}, 1 \mathrm{H}, 3-\underline{\mathrm{H}}_{2}\right), 1.56-1.44\left(\mathrm{~m}, 2 \mathrm{H}, 7-\mathrm{H}_{2}\right)$,

$1.49\left(\mathrm{~s}, 9 \mathrm{H}, 1-\mathrm{NH}-\mathrm{C}\left[\mathrm{NHCO}-\mathrm{OC}\left(\mathrm{CH}_{3}\right)_{3}\right]\left[\mathrm{NCO}-\mathrm{OC}\left(\mathrm{CH}_{3}\right)_{3}\right]\right)$

$1.49\left(\mathrm{~s}, 9 \mathrm{H}, 1-\mathrm{NH}-\mathrm{C}\left[\mathrm{NHCO}-\mathrm{OC}\left(\mathrm{C}_{3}\right)_{3}\right]\left[\mathrm{NCO}-\mathrm{OC}\left(\mathrm{CH}_{3}\right)_{3}\right]\right)$

1.44-1.30 (m, 2H, 6- $\left.\mathrm{H}_{2}\right), 0.90$ (s, 9H, 8-O-Si[ $\left.\left.\left(\mathrm{CH}_{3}\right)_{2}\right] \mathrm{C}\left(\mathrm{C}_{3}\right)_{3}\right)$,

0.05 (s, 6H, 8-O-Si[ $\left.\left.\left(\mathrm{C}_{3}\right)_{2}\right] \mathrm{C}\left(\mathrm{CH}_{3}\right)_{3}\right)$

${ }^{13} \mathrm{C}-\mathrm{NMR}\left(\mathbf{7 5 . 0} \mathrm{MHz} \mathrm{CDCl}_{3}\right)$

$\delta: 163.3\left(1 \mathrm{C}, 1-\mathrm{NH}-\mathrm{C}\left[\mathrm{NHCO}-\mathrm{OC}\left(\mathrm{CH}_{3}\right)_{3}\right]\left[\mathrm{NCO}-\mathrm{OC}\left(\mathrm{CH}_{3}\right)_{3}\right]\right)$, 
159.3 (1C, 4-O- $\left.-\mathrm{CH}_{2}-\mathrm{Ph}-\mathrm{OCH}_{3}\right)$,

$156.8\left(1 \mathrm{C}, 1-\mathrm{NH}-\underline{\mathrm{C}}\left[\mathrm{NHCO}-\mathrm{OC}\left(\mathrm{CH}_{3}\right)_{3}\right]\left[\mathrm{NCO}-\mathrm{OC}\left(\mathrm{CH}_{3}\right)_{3}\right]\right)$,

$153.0\left(1 \mathrm{C}, 1-\mathrm{NH}-\mathrm{C}\left[\mathrm{NHCO}-\mathrm{OC}\left(\mathrm{CH}_{3}\right)_{3}\right]\left[\mathrm{NCO}-\mathrm{OC}\left(\mathrm{CH}_{3}\right)_{3}\right]\right)$,

130.1 (1C, 4-O- $\left.-\mathrm{CH}_{2}-\mathrm{Ph}-\mathrm{OCH}_{3}\right)$,

129.5 (2C, 4-O- $\left.\mathrm{CH}_{2}-\mathrm{Ph}-\mathrm{OCH}_{3}\right), 113.9$ (2C, 4-O- $\left.\mathrm{CH}_{2}-\mathrm{Ph}-\mathrm{OCH}_{3}\right)$,

83.1 (1C, $\left.1-\mathrm{NH}-\mathrm{C}\left[\mathrm{NHCO}-\mathrm{OC}\left(\mathrm{CH}_{3}\right)_{3}\right]\left[\mathrm{NCO}-\mathrm{OC}\left(\mathrm{CH}_{3}\right)_{3}\right]\right)$,

78.7 (1C, 1-NH-C $\left.\left[\mathrm{NHCO}-\mathrm{OC}\left(\mathrm{CH}_{3}\right)_{3}\right]\left[\mathrm{NCO}-\mathrm{OC}\left(\mathrm{CH}_{3}\right)_{3}\right]\right), 78.7$ (C-4),

$70.2\left(1 \mathrm{C}, 3-\mathrm{O}-\mathrm{CH}_{2}-\mathrm{Ph}-\mathrm{OCH}_{3}\right), 70.0(\mathrm{C}-2), 63.0(\mathrm{C}-8), 55.3\left(1 \mathrm{C}, 4-\mathrm{O}-\mathrm{CH}_{2}-\mathrm{Ph}-\mathrm{OCH}_{3}\right)$,

47.0 (C-1), 38.4 (C-3), 33.2 (C-5), 33.0 (C-7),

28.3 (3C, 1-NH-C $\left.\left[\mathrm{NHCO}-\mathrm{OC}\left(\mathrm{CH}_{3}\right)_{3}\right]\left[\mathrm{NCO}-\mathrm{OC}\left(\mathrm{CH}_{3}\right)_{3}\right]\right)$

28.1 (3C, 1-NH-C $\left.\left[\mathrm{NHCO}-\mathrm{OC}\left(\mathrm{CH}_{3}\right)_{3}\right]\left[\mathrm{NCO}-\mathrm{OC}\left(\mathrm{CH}_{3}\right)_{3}\right]\right)$

26.0 (3C, 8-O-Si $\left.\left[\left(\mathrm{CH}_{3}\right)_{2}\right] \mathrm{C}\left(\mathrm{CH}_{3}\right)_{3}\right), 21.0$ (C-6),

$18.4\left(1 \mathrm{C}, 8-\mathrm{O}-\mathrm{Si}\left[\left(\mathrm{CH}_{3}\right)_{2}\right] \mathrm{C}\left(\mathrm{CH}_{3}\right)_{3}\right),-5.4\left(2 \mathrm{C}, 8-\mathrm{O}-\mathrm{Si}\left[\left(\mathrm{CH}_{3}\right)_{2}\right] \mathrm{C}\left(\mathrm{CH}_{3}\right)_{3}\right)$

HR-MS (FAB, NBA matrix)

$\mathbf{m} / \mathbf{z} 654.4158[\mathrm{M}+\mathrm{H}]^{+}$, Calcd for $\mathrm{C}_{33} \mathrm{H}_{60} \mathrm{~N}_{3} \mathrm{O}_{8} \mathrm{Si} 654.4150[\mathrm{M}+\mathrm{H}]$ 
(5S,4'R)-6-[N,3'-Bis(tert-butoxycarbonyl)-2'-iminoimidazolidin-4'-yl]-5-( $p$-methoxybe nzyloxy)hexyloxy-tert-butyldimethylsilane (+)-21

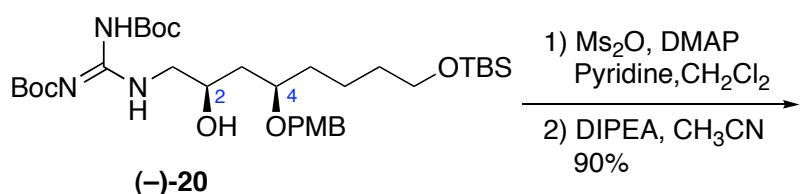

$(-)-20$

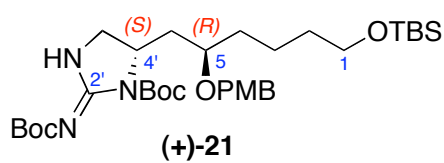

$(+)-21$

According to the procedure of preparation of the compound (-)-6, the title compound (+)-21 (176 mg, 90\%) was obtained from the (2R,4R)-guanidine alcohol (-)-20 (202 mg, $0.308 \mathrm{mmol})$.

$\mathbf{R} \boldsymbol{f}=0.25$ (Silica gel, Hexane $:$ EtOAc $=2: 1$ )

$[\alpha]_{\mathrm{D}}{ }^{24}+6.3\left(c 1.08, \mathrm{CHCl}_{3}\right)$

IR $(\mathrm{KBr}) \vee \mathrm{cm}^{-1}$

$1757(\mathrm{C}=\mathrm{O}), 1647(\mathrm{C}=\mathrm{N})$

\section{${ }^{1} \mathrm{H}-\mathrm{NMR}\left(\mathbf{2 7 0} \mathrm{MHz} \mathrm{CDCl}_{3}\right)$}

$\delta: 7.20\left(\mathrm{~d}, J=8.6 \mathrm{~Hz}, 2 \mathrm{H}, 5-\mathrm{O}-\mathrm{CH}_{2}-\mathrm{Ph}-\mathrm{OCH}_{3}\right)$,

$6.86\left(\mathrm{~d}, J=8.6 \mathrm{~Hz}, 2 \mathrm{H}, 5-\mathrm{O}-\mathrm{CH}_{2}-\mathrm{Ph}-\mathrm{OCH}_{3}\right)$,

$4.46\left(\mathrm{~d}, J=11.2 \mathrm{~Hz}, 1 \mathrm{H}, 5-\mathrm{O}-\mathrm{CH}_{2}-\mathrm{Ph}-\mathrm{OCH}_{3}\right)$,

4.32 (d, $\left.J=10.9 \mathrm{~Hz}, 1 \mathrm{H}, 5-\mathrm{O}-\underline{\mathrm{C}}_{2}-\mathrm{Ph}-\mathrm{OCH}_{3}\right), 4.12-4.02$ (m, 1H, 4'-H),

3.81-3.72 (m, 1H, 5'- $\left.\underline{\mathrm{H}}_{2}\right), 3.80\left(\mathrm{~s}, 3 \mathrm{H}, 5-\mathrm{O}-\mathrm{CH}_{2}-\mathrm{Ph}-\mathrm{OC}_{3}\right)$,

$3.60\left(\mathrm{t}, J=6.3 \mathrm{~Hz}, 2 \mathrm{H}, 1-\mathrm{H}_{2}\right), 3.62-3.54\left(\mathrm{~m}, 1 \mathrm{H}, 5\right.$ ' $\left.-\underline{\mathrm{H}}_{2}\right), 3.49-3.41(\mathrm{~m}, 1 \mathrm{H}, 5-\mathrm{H})$,

1.95-1.62 (m, 2H, 6- $\left.\underline{\mathrm{H}}_{2}\right), 1.57-1.34$ (complex m, 2H, 4- $\mathrm{H}_{2}$ ),

1.57-1.34 (complex m, 2H, 2- $\mathrm{H}_{2}$ ), 1.57-1.17 (complex m, 2H, 3- $\mathrm{H}_{2}$ ),

1.49 (s, 9H, 2'-NCO-OC $\left.\left(\mathrm{C}_{3}\right)_{3}\right), 1.48$ (s, 9H, 3'-CO-OC $\left.\left(\mathrm{C}_{3}\right)_{3}\right)$,

0.89 (s, 9H, 1-O-Si $\left.\left[\left(\mathrm{CH}_{3}\right)_{2}\right] \mathrm{C}\left(\mathrm{C}_{3}\right)_{3}\right), 0.05$ (s, 6H, 1-O-Si $\left.\left[\left(\mathrm{C}_{3}\right)_{2}\right] \mathrm{C}\left(\mathrm{CH}_{3}\right)_{3}\right)$

${ }^{13} \mathrm{C}-\mathrm{NMR}\left(67.5 \mathrm{MHz}, \mathrm{CDCl}_{3}\right)$

$\delta$ : $159.0\left(1 \mathrm{C}, 5-\mathrm{O}-\mathrm{CH}_{2}-\underline{\mathrm{Ph}}-\mathrm{OCH}_{3}\right), 130.3\left(1 \mathrm{C}, 5-\mathrm{O}-\mathrm{CH}_{2}-\underline{\mathrm{Ph}}-\mathrm{OCH}_{3}\right)$,

$129.2\left(2 \mathrm{C}, 5-\mathrm{O}-\mathrm{CH}_{2}-\mathrm{Ph}-\mathrm{OCH}_{3}\right), 113.6\left(2 \mathrm{C}, 5-\mathrm{O}-\mathrm{CH}_{2}-\mathrm{Ph}-\mathrm{OCH}_{3}\right)$,

82.7 (1C, 2'-NCO-O $\left.\underline{C}\left(\mathrm{CH}_{3}\right)_{3}\right), 78.7\left(1 \mathrm{C}, 3^{\prime}-\mathrm{CO}-\mathrm{O} \underline{\mathrm{C}}\left(\mathrm{CH}_{3}\right)_{3}\right)$

77.2 (C-5), 70.2 (1C, 5-O- $\left.\mathrm{CH}_{2}-\mathrm{Ph}-\mathrm{OCH}_{3}\right), 62.8$ (C-1), 56.9 (C-5'), 55.3 (C-4'),

55.1 (1C, 5-O- $\left.\mathrm{CH}_{2}-\mathrm{Ph}-\mathrm{OCH}_{3}\right), 38.6$ (C-6), 33.2 (C-4), 32.7 (C-2),

27.9 (3C, 2'-NCO-OC $\left.\left(\mathrm{CH}_{3}\right)_{3}\right), 27.9$ (3C, 3'-CO-O $\left.\underline{\mathrm{C}}\left(\mathrm{CH}_{3}\right)_{3}\right)$

$25.8\left(3 \mathrm{C}, 1-\mathrm{O}-\mathrm{Si}\left[\left(\mathrm{CH}_{3}\right)_{2}\right] \mathrm{C}\left(\mathrm{CH}_{3}\right)_{3}\right), 21.3(\mathrm{C}-3)$,

$18.2\left(1 \mathrm{C}, 1-\mathrm{O}-\mathrm{Si}\left[\left(\mathrm{CH}_{3}\right)_{2}\right] \underline{\mathrm{C}}\left(\mathrm{CH}_{3}\right)_{3}\right),-5.4\left(2 \mathrm{C}, 1-\mathrm{O}-\mathrm{Si}\left[\left(\mathrm{CH}_{3}\right)_{2}\right] \mathrm{C}\left(\mathrm{CH}_{3}\right)_{3}\right)$ 
Three signals of C-2', 2'-N $\underline{\mathrm{CO}}-\mathrm{OC}\left(\mathrm{CH}_{3}\right)_{3}$ and 3'- $\underline{\mathrm{CO}}-\mathrm{OC}\left(\mathrm{CH}_{3}\right)_{3}$ were not detected.

\section{HR-MS (FAB, NBA matrix)}

$\mathbf{m} / \mathbf{z} 636.4055[\mathrm{M}+\mathrm{H}]^{+}$, Calcd for $\mathrm{C}_{33} \mathrm{H}_{58} \mathrm{~N}_{3} \mathrm{O}_{7} \mathrm{Si} 636.4044[\mathrm{M}+\mathrm{H}]$ 
- $\quad\left(5 R, 4^{\prime} S\right)-6-\left[N, 3^{\prime}\right.$-Bis(tert-butoxycarbonyl)-2'-iminoimidazolidin-4'-yl]-5-( $p$ methoxybenzyloxy)hexan-1-ol (+)-VII

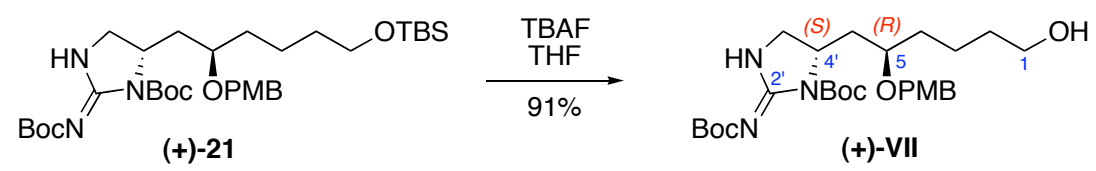

According to the procedure of preparation of the compound (-)-IV, the title compound (+)-VII (112 mg, 91\%) was obtained from the $\left(5 R, 4^{\prime} S\right)$-guanidine alcohol (+)-21 (151 mg, $0.237 \mathrm{mmol})$.

$\mathbf{R} \boldsymbol{f}=0.27$ (Silica gel, $\mathrm{CHCl}_{3}: \mathrm{MeOH}=10: 1$ )

$[\alpha]_{\mathrm{D}}{ }^{26}+14.7\left(c 1.53, \mathrm{CHCl}_{3}\right)$

IR $(\mathrm{KBr}) \vee \mathrm{cm}^{-1}$

$1755(\mathrm{C}=\mathrm{O}), 1651(\mathrm{C}=\mathrm{N})$

\section{${ }^{1} \mathrm{H}-\mathrm{NMR}\left(270 \mathrm{MHz}, \mathrm{CDCl}_{3}\right)$}

$\delta: 7.22\left(\mathrm{~d}, J=8.6 \mathrm{~Hz}, 2 \mathrm{H}, 5-\mathrm{O}-\mathrm{CH}_{2}-\mathrm{Ph}-\mathrm{OCH}_{3}\right)$,

$6.86\left(\mathrm{~d}, J=8.6 \mathrm{~Hz}, 2 \mathrm{H}, 5-\mathrm{O}-\mathrm{CH}_{2}-\mathrm{Ph}-\mathrm{OCH}_{3}\right)$,

$4.44\left(\mathrm{~d}, J=11.2 \mathrm{~Hz}, 1 \mathrm{H}, 5-\mathrm{O}-\mathrm{CH}_{2}-\mathrm{Ph}-\mathrm{OCH}_{3}\right)$,

$4.35\left(\mathrm{~d}, J=11.2 \mathrm{~Hz}, 1 \mathrm{H}, 5-\mathrm{O}-\underline{\mathrm{C}}_{2}-\mathrm{Ph}-\mathrm{OCH}_{3}\right), 4.05-3.98\left(\mathrm{~m}, 1 \mathrm{H}, 4^{\prime}-\mathrm{H}\right)$,

$3.80\left(\mathrm{~s}, 3 \mathrm{H}, 5-\mathrm{O}-\mathrm{CH}_{2}-\mathrm{Ph}-\mathrm{OCH}_{3}\right), 3.80-3.72\left(\mathrm{~m}, 1 \mathrm{H}, 5^{\prime}-\underline{\mathrm{H}}_{2}\right)$,

$3.64\left(\mathrm{t}, J=6.3 \mathrm{~Hz}, 2 \mathrm{H}, 1-\mathrm{H}_{2}\right), 3.66-3.58\left(\mathrm{~m}, 1 \mathrm{H}, 5^{\prime}-\underline{\mathrm{H}}_{2}\right), 3.44-3.40(\mathrm{~m}, 1 \mathrm{H}, 5-\mathrm{H})$,

1.96-1.77 (m, 2H, 6- $\mathrm{H}_{2}$ ), 1.72-1.54 (m, 2H, 4- $\mathrm{H}_{2}$ ), 1.57-1.34 (complex m, 2H, 2- $\mathrm{H}_{2}$ ),

1.57-1.34 (complex m, 2H, 3- $\mathrm{H}_{2}$ ), 1.49 (s, 9H, 2'-NCO-OC $\left.\left(\mathrm{C}_{3}\right)_{3}\right)$,

1.47 (s, 9H, 3'-CO-OC $\left.\left(\mathrm{C}_{3}\right)_{3}\right)$,

${ }^{13} \mathrm{C}-\mathrm{NMR}\left(67.5 \mathrm{MHz}, \mathrm{CDCl}_{3}\right)$

$\delta$ : $159.0\left(1 \mathrm{C}, 5-\mathrm{O}-\mathrm{CH}_{2}-\mathrm{Ph}-\mathrm{OCH}_{3}\right), 130.2\left(1 \mathrm{C}, 5-\mathrm{O}-\mathrm{CH}_{2}-\mathrm{Ph}-\mathrm{OCH}_{3}\right)$, $129.2\left(2 \mathrm{C}, 5-\mathrm{O}-\mathrm{CH}_{2}-\mathrm{Ph}-\mathrm{OCH}_{3}\right), 113.6\left(2 \mathrm{C}, 5-\mathrm{O}-\mathrm{CH}_{2}-\mathrm{Ph}-\mathrm{OCH}_{3}\right)$, $82.8\left(1 \mathrm{C}, 2^{\prime}-\mathrm{NCO}-\mathrm{O} \underline{\mathrm{C}}\left(\mathrm{CH}_{3}\right)_{3}\right), 80.3\left(1 \mathrm{C}, 3\right.$ '- $\left.\mathrm{CO}-\mathrm{O} \underline{\mathrm{C}}\left(\mathrm{CH}_{3}\right)_{3}\right)$ 76.0 (C-5), 70.2 (1C, 5-O- $\mathrm{CH}_{2}-\mathrm{Ph}-\mathrm{OCH}_{3}$ ), 62.3 (C-1), 55.2 (C-4'), 55.1 (C-5'), 55.1 (1C, 5-O-CH$\left.-\mathrm{Ph}-\mathrm{OCH}_{3}\right), 38.5$ (C-6), 33.4 (C-4), 32.5 (C-2), 28.0 (3C, 2'-NCO-OC( $\left.\left(\mathrm{CH}_{3}\right)_{3}\right), 27.9$ (3C, 3'-CO-OC $\left.\left(\mathrm{CH}_{3}\right)_{3}\right), 21.1$ (C-3),

Three signals of C-2', 2'-NCO-OC( $\left.\mathrm{CH}_{3}\right)_{3}$ and 3'- $\underline{\mathrm{CO}}-\mathrm{OC}\left(\mathrm{CH}_{3}\right)_{3}$ were not detected. HR-MS (FAB, NBA matrix) $\mathbf{m} / \mathbf{z} 522.3168[\mathrm{M}+\mathrm{H}]^{+}$, Calcd for $\mathrm{C}_{27} \mathrm{H}_{44} \mathrm{~N}_{3} \mathrm{O}_{7} 522.3179[\mathrm{M}+\mathrm{H}]$ 
- $\quad\left(5 R, 4^{\prime} S\right)-6-\left[N, 3^{\prime}\right.$-Bis (tert-butoxycarbonyl)-2'-iminoimidazolidin-4'-yl]-5-( $p$ methoxybenzyloxy)hexanal (+)-VIII

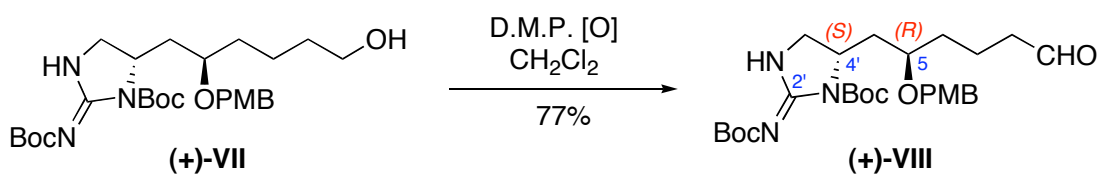

According to the procedure of preparation of the compound (-)-18, the title compound (+)-VIII (81.0 mg, 77\%) was obtained from the (5R, 4'S)-primary alcohol (+)-VII (106 mg, $0.237 \mathrm{mmol})$.

$\mathbf{R} \boldsymbol{f}=0.46$ (Silica gel, $\left.\mathrm{CHCl}_{3}: \mathrm{MeOH}=10: 1\right)$

$[\alpha]_{\mathbf{D}}^{29}+20.0\left(c 0.55, \mathrm{CHCl}_{3}\right)$

IR $(\mathrm{KBr}) \vee \mathrm{cm}^{-1}$

1754, $(\mathrm{C}=\mathrm{O}), 1649(\mathrm{C}=\mathrm{N})$

\section{${ }^{1} \mathrm{H}-\mathrm{NMR}\left(270 \mathrm{MHz}, \mathrm{CDCl}_{3}\right)$}

$\delta$ : $9.72(\mathrm{~s}, 1 \mathrm{H}, 1-\mathrm{H}), 7.19\left(\mathrm{~d}, J=8.6 \mathrm{~Hz}, 2 \mathrm{H}, 5-\mathrm{O}-\mathrm{CH}_{2}-\mathrm{Ph}-\mathrm{OCH}_{3}\right)$,

$6.84\left(\mathrm{~d}, J=8.6 \mathrm{~Hz}, 2 \mathrm{H}, 5-\mathrm{O}-\mathrm{CH}_{2}-\mathrm{Ph}-\mathrm{OCH}_{3}\right)$,

$4.40\left(\mathrm{~d}, J=11.2 \mathrm{~Hz}, 1 \mathrm{H}, 5-\mathrm{O}-\mathrm{CH}_{2}-\mathrm{Ph}-\mathrm{OCH}_{3}\right)$,

4.33 (d, $\left.J=11.2 \mathrm{~Hz}, 1 \mathrm{H}, 5-\mathrm{O}-\mathrm{C}_{2}-\mathrm{Ph}-\mathrm{OCH}_{3}\right), 4.03-3.93$ (m, 1H, 4'-H),

3.77 (s, 3H, 5-O- $\left.\mathrm{CH}_{2}-\mathrm{Ph}-\mathrm{OC}_{\underline{H}}\right)$, 3.77-3.67 (m, 1H, 5'- $\left.\underline{\mathrm{H}}_{2}\right), 3.62-3.50\left(\mathrm{~m}, 1 \mathrm{H}, 5^{\prime}-\underline{\mathrm{H}}_{2}\right)$,

3.43-3.37 (m, 1H, 5-H), $2.41\left(\mathrm{t}, J=6.4 \mathrm{~Hz}, 2 \mathrm{H}, 2-\mathrm{H}_{2}\right), 1.97-1.87\left(\mathrm{~m}, 1 \mathrm{H}, 6-\underline{\mathrm{H}}_{2}\right)$,

1.81-1.71 (m, 1H, 6- $\underline{\mathrm{H}}_{2}$ ), 1.65-1.50 (complex m, 2H, 3- $\mathrm{H}_{2}$ ),

1.65-1.50 (complex m, 2H, 4- $\left.\mathrm{H}_{2}\right), 1.46$ (s, 9H, 2'-NCO-OC $\left.\left(\mathrm{CH}_{3}\right)_{3}\right)$,

1.45 (s, 9H, 3'-CO-OC $\left.\left(\mathrm{C}_{3}\right)_{3}\right)$,

${ }^{13} \mathrm{C}-\mathrm{NMR}\left(67.5 \mathrm{MHz}, \mathrm{CDCl}_{3}\right)$

$\delta$ : 201.8 (C-1), 159.1 (1C, 5-O- $\left.\mathrm{CH}_{2}-\mathrm{Ph}-\mathrm{OCH}_{3}\right), 130.4$ (1C, 5-O- $\left.\mathrm{CH}_{2}-\mathrm{Ph}-\mathrm{OCH}_{3}\right)$, $129.3\left(2 \mathrm{C}, 5-\mathrm{O}-\mathrm{CH}_{2}-\mathrm{Ph}-\mathrm{OCH}_{3}\right), 113.7$ (2C, 5-O- $\left.\mathrm{CH}_{2}-\mathrm{Ph}-\mathrm{OCH}_{3}\right)$,

82.9 (1C, 2'-NCO-O $\left.\underline{\mathrm{C}}\left(\mathrm{CH}_{3}\right)_{3}\right), 81.1$ (1C, 3'-CO-O $\left.\underline{\mathrm{C}}\left(\mathrm{CH}_{3}\right)_{3}\right)$

75.4 (C-5), $70.3\left(1 \mathrm{C}, 5-\mathrm{O}-\underline{\mathrm{CH}}_{2}-\mathrm{Ph}-\mathrm{OCH}_{3}\right), 55.1$ (1C, 5-O- $\left.\mathrm{CH}_{2}-\mathrm{Ph}-\mathrm{O}^{-} \mathrm{H}_{3}\right), 55.1(\mathrm{C}-4$ '), 55.1 (C-5'), 43.6 (C-2), 38.3 (C-6), 33.0 (C-4), 28.1 (3C, 2'-NCO-OC $\left.\left(\underline{\mathrm{CH}}_{3}\right)_{3}\right)$, 27.9 (3C, 3'-CO-O $\left.\underline{\mathrm{C}}\left(\mathrm{CH}_{3}\right)_{3}\right), 17.3(\mathrm{C}-3)$,

Three signals of C-2', 2'-NCO-OC( $\left(\mathrm{CH}_{3}\right)_{3}$ and 3'- $\mathrm{CO}-\mathrm{OC}\left(\mathrm{CH}_{3}\right)_{3}$ were not detected.

HR-MS (FAB, NBA matrix)

$\mathbf{m} / \mathbf{z} 542.2823[\mathrm{M}+\mathrm{Na}]^{+}$, Calcd for $\mathrm{C}_{27} \mathrm{H}_{41} \mathrm{~N}_{3} \mathrm{O}_{7} \mathrm{Na} 542.2842[\mathrm{M}+\mathrm{Na}]$ 
- (5R,4'S)-6-[N,3'-Bis(tert-butoxycarbonyl)-1'-( $p$-methoxyphenylcarbamoyl)-2'iminoimidazolidin-4'-yl]-5-(p-methoxybenzyloxy)hexanal (+)-IX

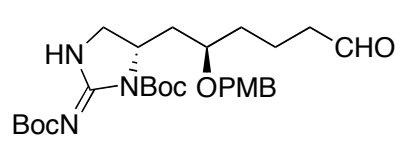

(+)-VIII
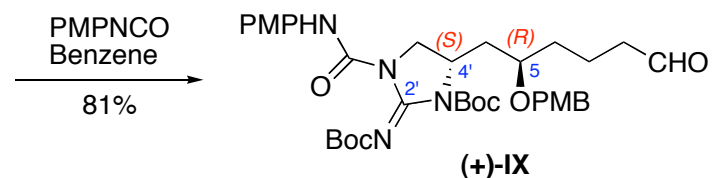

According to the procedure of preparation of the compound (-)-V, the title compound (+)-IX $(66.8 \mathrm{mg}, 81 \%)$ was obtained from the (5R, 4'S)-aldehyde (+)-VIII $(64.0 \mathrm{mg}$, $0.123 \mathrm{mmol})$.

$\mathbf{R} f=0.53$ (Silica gel, $\mathrm{CHCl}_{3}: \mathrm{MeOH}=10: 1$ )

$[\alpha]_{\mathrm{D}}{ }^{24}+50.1\left(c 0.54, \mathrm{CHCl}_{3}\right)$

IR $(\mathrm{KBr}) v \mathrm{~cm}^{-1}$

$1755(\mathrm{C}=\mathrm{O}), 1693(\mathrm{C}=\mathrm{N})$

\section{${ }^{1} \mathrm{H}-\mathrm{NMR}\left(270 \mathrm{MHz}, \mathrm{CDCl}_{3}\right)$}

$\delta: 11.0\left(\mathrm{~s}, 1 \mathrm{H}, 1^{\prime}-\mathrm{CO}-\mathrm{NH}-\mathrm{Ph}-\mathrm{OCH}_{3}\right), 9.74(\mathrm{~s}, 1 \mathrm{H}, 1-\mathrm{H})$,

7.37 (d, $J=8.6 \mathrm{~Hz}, 2 \mathrm{H}, 1^{\prime}-\mathrm{CO}-\mathrm{NH}-\underline{\mathrm{Ph}}-\mathrm{OCH}_{3}$ ),

$7.24\left(\mathrm{~d}, J=8.9 \mathrm{~Hz}, 2 \mathrm{H}, 5-\mathrm{O}-\mathrm{CH}_{2}-\mathrm{Ph}-\mathrm{OCH}_{3}\right)$,

$6.87\left(\mathrm{~d}, J=8.6 \mathrm{~Hz}, 2 \mathrm{H}, 1^{\prime}-\mathrm{CO}-\mathrm{NH}-\mathrm{Ph}-\mathrm{OCH}_{3}\right)$,

$6.86\left(\mathrm{~d}, J=8.9 \mathrm{~Hz}, 2 \mathrm{H}, 5-\mathrm{O}-\mathrm{CH}_{2}-\mathrm{Ph}-\mathrm{OCH}_{3}\right)$,

4.47 (d, $J=11.2 \mathrm{~Hz}, 1 \mathrm{H}, 5-\mathrm{O}-\mathrm{C}_{2}-\mathrm{Ph}-\mathrm{OCH}_{3}$ ),

$4.36\left(\mathrm{~d}, J=11.2 \mathrm{~Hz}, 1 \mathrm{H}, 5-\mathrm{O}-\mathrm{C}_{2}-\mathrm{Ph}-\mathrm{OCH}_{3}\right), 4.09-4.05\left(\mathrm{~m}, 1 \mathrm{H}, 5^{\prime}-\underline{-}_{2}\right)$,

4.05-4.00 (m, 1H, 4'-H), 3.78 (s, 3H, 1'-CO-NH-Ph-OC튼 ${ }_{3}$ ),

$3.78\left(\mathrm{~s}, 3 \mathrm{H}, 5-\mathrm{O}-\mathrm{CH}_{2}-\mathrm{Ph}-\mathrm{OC}_{\underline{3}}\right), 3.78-3.74\left(\mathrm{~m}, 1 \mathrm{H}, 5\right.$ ' $\left.-\underline{\mathrm{H}}_{2}\right)$,

3.54-3.46 (m, 1H, 5-H), $2.44\left(\mathrm{dt}, J=1.3,6.9 \mathrm{~Hz}, 2 \mathrm{H}, 2-\mathrm{H}_{2}\right)$,

1.97-1.92 (m, 2H, 6- $\left.\mathrm{H}_{2}\right), 1.70-1.56\left(\mathrm{~m}, 2 \mathrm{H}, 3-\mathrm{H}_{2}\right), 1.70-1.56\left(\mathrm{~m}, 2 \mathrm{H}, 4-\mathrm{H}_{2}\right)$,

1.52 (s, 9H, 2'-NCO-OC( $\left.\left.\mathrm{C}_{3}\right)_{3}\right), 1.48$ (s, 9H, 3'-CO-OC $\left.\left(\underline{\mathrm{C}}_{3}\right)_{3}\right)$,

\section{${ }^{13} \mathrm{C}-\mathrm{NMR}\left(67.5 \mathrm{MHz}, \mathrm{CDCl}_{3}\right)$}

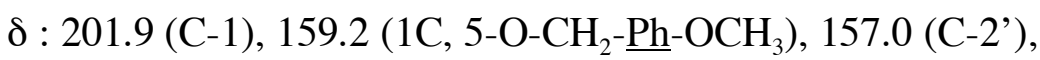

156.3 (1C, 1'-CO-NH-Ph-OCH $\left.{ }_{3}\right), 150.3\left(1 \mathrm{C}, 2^{\prime}\right.$ - $\left.\mathrm{NCO}-\mathrm{OC}\left(\mathrm{CH}_{3}\right)_{3}\right)$,

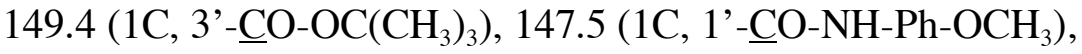

130.5 (1C, 1'-CO-NH-Ph-OCH ${ }_{3}$, 130.0 (1C, 5-O- $\left.\mathrm{CH}_{2}-\mathrm{Ph}-\mathrm{OCH}_{3}\right)$, $129.4\left(2 \mathrm{C}, 5-\mathrm{O}-\mathrm{CH}_{2}-\mathrm{Ph}-\mathrm{OCH}_{3}\right), 122.2$ (2C, 1'-CO-NH-Ph-OCH ${ }_{3}$ ), $114.1\left(2 \mathrm{C}, 1^{\prime}-\mathrm{CO}-\mathrm{NH}-\mathrm{Ph}-\mathrm{OCH}_{3}\right)$, 
$113.8\left(2 \mathrm{C}, 5-\mathrm{O}-\mathrm{CH}_{2}-\mathrm{Ph}-\mathrm{OCH}_{3}\right), 83.9\left(1 \mathrm{C}, 2^{\prime}-\mathrm{NCO}-\mathrm{O} \underline{\mathrm{C}}\left(\mathrm{CH}_{3}\right)_{3}\right)$,

80.5 (1C, 3'-CO-OC $\left.\left(\mathrm{CH}_{3}\right)_{3}\right), 75.4$ (C-5), 70.1 (1C, 5- $\left.-\mathrm{C}^{-} \mathrm{H}_{2}-\mathrm{Ph}-\mathrm{OCH}_{3}\right)$, 55.4 (1C, 1'-CO-NH-Ph-OCH ${ }_{3}$ ), 55.2 (1C, 5-O- $\left.\mathrm{CH}_{2}-\mathrm{Ph}_{-} \mathrm{OCH}_{3}\right), 53.9$ (C-4'),

47.2 (C-5'), 43.6 (C-2), 38.8 (C-6), 32.7 (C-4),28.0 (3C, 2'-NCO-OC( $\left.\left(\mathrm{CH}_{3}\right)_{3}\right)$, $28.0\left(3 \mathrm{C}, 3^{\prime}-\mathrm{CO}-\mathrm{O} \underline{\mathrm{C}}\left(\mathrm{CH}_{3}\right)_{3}\right), 17.2(\mathrm{C}-3)$

\section{HR-MS (FAB, NBA matrix)}

$\mathbf{m} / \mathbf{z} 669.3510[\mathrm{M}+\mathrm{H}]^{+}$, Calcd for $\mathrm{C}_{35} \mathrm{H}_{49} \mathrm{~N}_{4} \mathrm{O}_{9} 669.3500[\mathrm{M}+\mathrm{H}]$ 
- (5R,4'S)-6-[N,3'-Bis(tert-butoxycarbonyl)-1'-( $p$-methoxyphenylcarbamoyl)-2'iminoimidazolidin-4'-yl]-5-(p-methoxybenzyloxy)hexanoic acid (+)-22
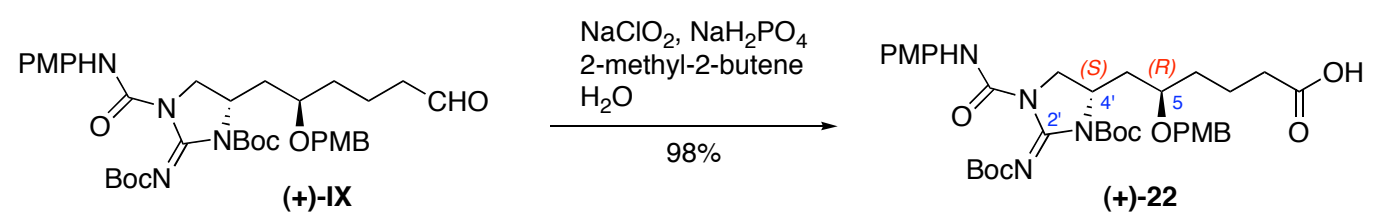

According to the procedure of preparation of the compound (-)-19, the title compound (+)-22 (60.7 mg, 98\%) was obtained from the (5R, 4'S)-aldehyde (+)-IX (60.2 mg, $0.090 \mathrm{mmol}$ ).

$\mathbf{R} \boldsymbol{f}=0.37$ (Silica gel, $\mathrm{CHCl}_{3}: \mathrm{MeOH}=10: 1$ )

$[\alpha]_{D}{ }^{24}+45.0\left(c 0.64, \mathrm{CHCl}_{3}\right)$

IR $(\mathrm{KBr}) v \mathrm{~cm}^{-1}$

$1751(\mathrm{C}=\mathrm{O}), 1702(\mathrm{C}=\mathrm{N})$

\section{${ }^{1} \mathrm{H}-\mathrm{NMR}\left(270 \mathrm{MHz}, \mathrm{CDCl}_{3}\right)$}

$\delta: 11.0$ (s, 1H, 1'-CO-N $\left.\underline{\mathrm{H}}-\mathrm{Ph}-\mathrm{OCH}_{3}\right), 7.37$ (d, $J=8.6 \mathrm{~Hz}, 2 \mathrm{H}, 1^{\prime}-\mathrm{CO}-\mathrm{NH}-\underline{\mathrm{Ph}}-\mathrm{OCH}_{3}$ ),

$7.24\left(\mathrm{~d}, J=8.6 \mathrm{~Hz}, 2 \mathrm{H}, 5-\mathrm{O}-\mathrm{CH}_{2}-\mathrm{Ph}-\mathrm{OCH}_{3}\right)$,

$6.87\left(\mathrm{~d}, J=8.6 \mathrm{~Hz}, 2 \mathrm{H}, 5-\mathrm{O}-\mathrm{CH}_{2}-\mathrm{Ph}-\mathrm{OCH}_{3}\right)$,

$6.86\left(\mathrm{~d}, J=8.6 \mathrm{~Hz}, 2 \mathrm{H}, 1^{\prime}-\mathrm{CO}-\mathrm{NH}-\mathrm{Ph}-\mathrm{OCH}_{3}\right)$,

$4.48\left(\mathrm{~d}, J=11.2 \mathrm{~Hz}, 1 \mathrm{H}, 5-\mathrm{O}-\mathrm{C}_{2}-\mathrm{Ph}-\mathrm{OCH}_{3}\right.$ ),

$4.36\left(\mathrm{~d}, J=11.2 \mathrm{~Hz}, 1 \mathrm{H}, 5-\mathrm{O}-\mathrm{C}_{2}-\mathrm{Ph}-\mathrm{OCH}_{3}\right), 4.09-4.05\left(\mathrm{~m}, 1 \mathrm{H}, 5^{\prime}-\underline{-}_{2}\right)$,

4.07-4.00 (m, 1H, 4'-H), 3.78 (s, $3 \mathrm{H}, 5-\mathrm{O}-\mathrm{CH}_{2}-\mathrm{Ph}-\mathrm{OCH}_{3}$ ),

3.78 (s, 3H, 1'-CO-NH-Ph-OC些), 3.78-3.74 (m, 1H, 5'- $\left.\underline{\mathrm{H}}_{2}\right)$,

3.54-3.47 (m, 1H, 5-H), 2.35 (t, $\left.J=6.6 \mathrm{~Hz}, 2 \mathrm{H}, 2-\mathrm{H}_{2}\right)$,

1.96-1.92 (m, 2H, 6- $\left.\mathrm{H}_{2}\right), 1.69-1.57$ (m, 2H, 3- $\left.\mathrm{H}_{2}\right), 1.69-1.57$ (m, 2H, 4- $\mathrm{H}_{2}$ ),

1.52 (s, 9H, 2'-NCO-OC( $\left.\left(\underline{\mathrm{H}}_{3}\right)_{3}\right), 1.47$ (s, 9H, 3'-CO-OC( $\left.\left.\mathrm{CH}_{3}\right)_{3}\right)$

\section{${ }^{13} \mathrm{C}-\mathrm{NMR}\left(67.5 \mathrm{MHz}, \mathrm{CDCl}_{3}\right)$}

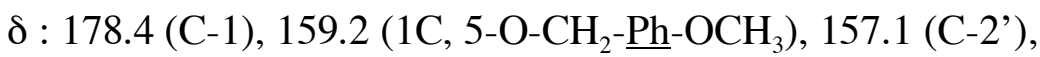

156.3 (1C, 1'-CO-NH-Ph-OCH $\left.{ }_{3}\right), 150.3\left(1 \mathrm{C}, 2^{\prime}\right.$ - $\left.\mathrm{NCO}-\mathrm{OC}\left(\mathrm{CH}_{3}\right)_{3}\right)$,

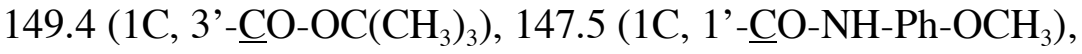

130.5 (1C, 1'-CO-NH-Ph- $\left.-\mathrm{OCH}_{3}\right), 130.0$ (1C, 5-O- $\left.\mathrm{CH}_{2}-\mathrm{Ph}-\mathrm{OCH}_{3}\right)$,

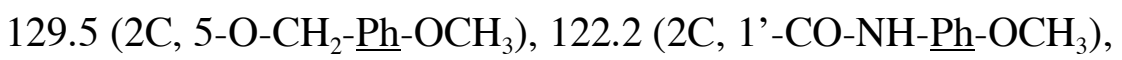

114.1 (2C, 1'-CO-NH- $\left.\mathrm{Ph}-\mathrm{OCH}_{3}\right), 113.8$ (2C, 5-O- $\left.\mathrm{CH}_{2}-\mathrm{Ph}-\mathrm{OCH}_{3}\right)$, 
84.0 (1C, 2'-NCO-O $\left.\underline{\mathrm{C}}\left(\mathrm{CH}_{3}\right)_{3}\right), 80.6$ (1C, 3'-CO-O $\left.\underline{\mathrm{C}}\left(\mathrm{CH}_{3}\right)_{3}\right)$,

75.3 (C-5), 70.1 (1C, 5-O- $\mathrm{CH}_{2}-\mathrm{Ph}-\mathrm{OCH}_{3}$ ), 55.4 (1C, 1'-CO-NH-Ph-OCH${ }_{3}$ ),

55.2 (1C, 5-O- $\mathrm{CH}_{2}-\mathrm{Ph}-\mathrm{OCH}_{3}$ ), 53.8 (C-4'), 47.2 (C-5'), 38.8 (C-6), 33.7 (C-2),

32.6 (C-4), 28.0 (3C, 2'-NCO-OC $\left.\left(\mathrm{CH}_{3}\right)_{3}\right), 28.0$ (3C, 3'-CO-O $\left.\underline{\mathrm{C}}\left(\mathrm{CH}_{3}\right)_{3}\right), 19.8$ (C-3)

HR-MS (FAB, NBA matrix)

$\mathbf{m} / \mathbf{z} 685.3483[\mathrm{M}+\mathrm{H}]^{+}$, Calcd for $\mathrm{C}_{35} \mathrm{H}_{49} \mathrm{~N}_{4} \mathrm{O}_{9} 685.3449[\mathrm{M}+\mathrm{H}]$ 
- $\quad\left(5 R, 4^{\prime} S\right)-6-\left[N, 3^{\prime}\right.$-Bis(tert-butoxycarbonyl)-1'-carbamoyl-2'-iminoimidazolidin4'-yl]-5-( $p$-methoxybenzyloxy)hexanoic acid (+)-X

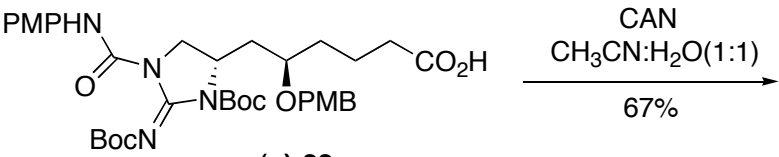

$(+)-22$

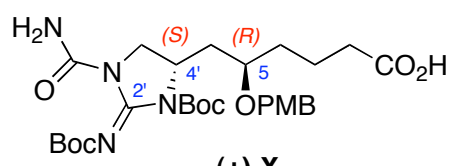

$(+)-\mathbf{X}$

According to the procedure of preparation of the compound (-)-VI, the title compound (+)-X (30.0 mg, 67\%) was obtained from the (5R, 4' $S$ )-carboxylic acid (+)-22 (52.5 mg, $0.077 \mathrm{mmol})$.

$\mathbf{R} \boldsymbol{f}=0.27$ (Silica gel, $\mathrm{CHCl}_{3}: \mathrm{MeOH}=10: 1$ )

$[\alpha]_{\mathrm{D}}^{25}+20.5\left(c 1.42, \mathrm{CHCl}_{3}\right)$

IR $(\mathrm{KBr}) \vee \mathrm{cm}^{-1}$

$1753(\mathrm{C}=\mathrm{O}), 1707(\mathrm{C}=\mathrm{N})$

\section{${ }^{1} \mathrm{H}-\mathrm{NMR}\left(270 \mathrm{MHz} \mathrm{CDCl}_{3}\right)$}

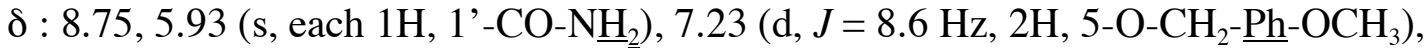

$6.87\left(\mathrm{~d}, J=8.6 \mathrm{~Hz}, 2 \mathrm{H}, 5-\mathrm{O}-\mathrm{CH}_{2}-\mathrm{Ph}-\mathrm{OCH}_{3}\right)$,

$4.47\left(\mathrm{~d}, J=11.5 \mathrm{~Hz}, 1 \mathrm{H}, 5-\mathrm{O}-\mathrm{CH}_{2}-\mathrm{Ph}-\mathrm{OCH}_{3}\right)$,

4.35 (d, $J=11.5 \mathrm{~Hz}, 1 \mathrm{H}, 5-\mathrm{O}-\mathrm{C}_{2}-\mathrm{Ph}-\mathrm{OCH}_{3}$ ), 3.99-3.95 (complex m, 1H, 4'-H),

3.99-3.95 (complex m, 1H, 5'- $\underline{\mathrm{H}}_{2}$ ), 3.79 (s, 3H, 5-O- $\mathrm{CH}_{2}-\mathrm{Ph}-\mathrm{OC}_{\underline{3}}$ ),

$3.70\left(\mathrm{dd}, J=8.3,11.2 \mathrm{~Hz}, 1 \mathrm{H}, 5\right.$ ' $\left.-\underline{\mathrm{H}}_{2}\right), 3.52-3.43(\mathrm{~m}, 1 \mathrm{H}, 5-\mathrm{H})$,

$2.34\left(\mathrm{t}, J=6.3 \mathrm{~Hz}, 2 \mathrm{H}, 2-\mathrm{H}_{2}\right), 1.93-1.88\left(\mathrm{~m}, 2 \mathrm{H}, 6-\mathrm{H}_{2}\right)$

1.69-1.55 (complex m, 2H, 3- $\mathrm{H}_{2}$ ), 1.69-1.55 (complex m, 2H, 4- $\mathrm{H}_{2}$ ),

1.49 (s, 9H, 2'-NCO-OC( $\left.\left(\underline{\mathrm{H}}_{3}\right)_{3}\right), 1.46$ (s, 9H, 3'-CO-OC $\left.\left(\mathrm{C}_{3}\right)_{3}\right)$

${ }^{13} \mathrm{C}-\mathrm{NMR}\left(67.5 \mathrm{MHz} \mathrm{CDCl}_{3}\right)$

$\delta$ : 177.7 (C-1), $159.2\left(1 \mathrm{C}, 5-\mathrm{O}-\mathrm{CH}_{2}-\mathrm{Ph}-\mathrm{OCH}_{3}\right), 157.3$ (C-2'),

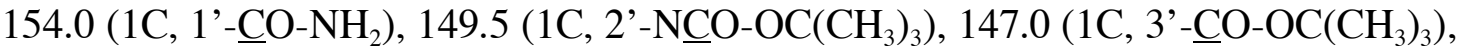

$130.1\left(1 \mathrm{C}, 5-\mathrm{O}-\mathrm{CH}_{2}-\mathrm{Ph}-\mathrm{OCH}_{3}\right), 129.5\left(2 \mathrm{C}, 5-\mathrm{O}-\mathrm{CH}_{2}-\mathrm{Ph}-\mathrm{OCH}_{3}\right)$,

113.8 (2C, 5-O- $\left.\mathrm{CH}_{2}-\underline{\mathrm{Ph}}-\mathrm{OCH}_{3}\right), 83.9$ (1C, 2'-NCO-OC $\left.\left(\mathrm{CH}_{3}\right)_{3}\right)$,

80.6 (1C, 3'-CO-O $\left.\underline{C}\left(\mathrm{CH}_{3}\right)_{3}\right), 75.3$ (C-5), 70.8 (1C, 5-O- $\left.\underline{\mathrm{CH}}_{2}-\mathrm{Ph}-\mathrm{OCH}_{3}\right)$,

55.2 (1C, 5-O- $\mathrm{CH}_{2}-\mathrm{Ph}-\mathrm{OCH}_{3}$ ), 53.9 (C-4'), 47.1 (C-5'), 38.8 (C-6), 33.7 (C-2),

32.7 (C-4), 28.0 (3C, 2'-NCO-OC $\left.\left(\underline{\mathrm{CH}}_{3}\right)_{3}\right), 28.0$ (3C, 3'-CO-O $\left.\underline{\mathrm{C}}\left(\mathrm{CH}_{3}\right)_{3}\right), 19.9$ (C-3)

\section{HR-MS (FAB, NBA matrix)}

$\mathbf{m} / \mathbf{z} 579.3032[\mathrm{M}+\mathrm{H}]^{+}$, Calcd for $\mathrm{C}_{28} \mathrm{H}_{43} \mathrm{~N}_{4} \mathrm{O}_{9} 579.3030[\mathrm{M}+\mathrm{H}]$ 
- (5R,4'S)-6-(1'-Carbamoyl-2'-iminoimidazolidin-4'-yl)-5-hydroxyhexanoic acid (-)-(5R, 4'S)-K01-0509 B (10)



$(+)-X$

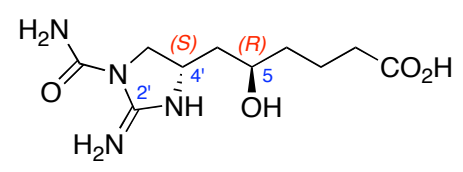

(-)-(5R, 4'S)-K01-0509 B (10)

According to the procedure of preparation of the compound (+)-(5R, $\left.4^{\prime} R\right)-\mathrm{K} 01-0509 \mathrm{~B}$ (5), the title compound (-)-(5R, 4'S)-K01-0509 B (10) (4.6 mg, 39\%) was obtained from the (5R,4'S)-carboxylic acid (+)-X (26.3 mg, $0.045 \mathrm{mmol})$.

$[\alpha]_{\mathbf{D}}{ }^{26}-33.9(c 0.21, \mathrm{MeOH})$

IR $(\mathrm{KBr}) \vee \mathrm{cm}^{-1}$

$1730(\mathrm{C}=\mathrm{O}), 1678(\mathrm{C}=\mathrm{N})$

\section{${ }^{1} \mathrm{H}-\mathrm{NMR}\left(400 \mathrm{MHz}, \mathrm{D}_{2} \mathrm{O}\right)$}

$\delta$ : 4.26-4.21 (m, 1H, 4'-H), $4.22\left(\mathrm{dd}, J=9.0,14.0 \mathrm{~Hz}, 1 \mathrm{H}, 5^{\prime}-\underline{\mathrm{H}}_{2}\right)$

$3.77\left(\mathrm{dd}, J=2.5,14.0 \mathrm{~Hz}, 1 \mathrm{H}, 5^{\prime}-\underline{\mathrm{H}}_{2}\right), 3.77-3.71(\mathrm{~m}, 1 \mathrm{H}, 5-\mathrm{H})$,

$2.33\left(\mathrm{t}, J=7.2 \mathrm{~Hz}, 2 \mathrm{H}, 2-\mathrm{H}_{2}\right), 1.92-1.87\left(\mathrm{~m}, 1 \mathrm{H}, 6-\underline{\mathrm{H}}_{2}\right)$,

1.86 (ddd, $\left.J=3.0,6.5,13.5 \mathrm{~Hz}, 1 \mathrm{H}, 6-\underline{\mathrm{H}}_{2}\right), 1.76-1.65\left(\mathrm{~m}, 1 \mathrm{H}, 3-\underline{\mathrm{H}}_{2}\right)$,

1.63-1.53 (m, 1H, 3- $\left.\underline{\mathrm{H}}_{2}\right), 1.51-1.45\left(\mathrm{~m}, 2 \mathrm{H}, 4-\mathrm{H}_{2}\right)$

${ }^{13} \mathrm{C}-\mathrm{NMR}\left(75 \mathrm{MHz}, \mathrm{D}_{2} \mathrm{O}\right)$

$\delta$ : 180.1 (C-1), 156.5 (1C, 1'- $\underline{\text { CO-NH }}$ ), 156.0 (2'-C), 68.0 (5-C), 51.2 (4'-C),

50.8 (5'-C), 41.3 (6-C), 36.2 (4-C), 34.5 (2-C), 20.9 (3-C)

\section{HR-MS (FAB, NBA matrix)}

$\mathbf{m} / \mathbf{z} 259.1411[\mathrm{M}+\mathrm{H}]^{+}$, Calcd for $\mathrm{C}_{10} \mathrm{H}_{19} \mathrm{~N}_{4} \mathrm{O}_{4} 259.1406[\mathrm{M}+\mathrm{H}]$

\section{${ }^{1} \mathrm{H}$ - and ${ }^{13} \mathrm{C}$-NMR is not pure due to the instability of this diastereomer.}

